\title{
Tie-Breaking Strategies for Cost-Optimal Best First Search
}

\author{
Masataro Asai \\ Alex Fukunaga \\ Graduate School of Arts and Sciences \\ The University of Tokyo \\ Japan
}

\author{
GUICHO2.71828@GMAIL.COM \\ FUKUNAGA@IDEA.C.U-TOKYO.AC.JP
}

\begin{abstract}
Best-first search algorithms such as A* need to apply tie-breaking strategies in order to decide which node to expand when multiple search nodes have the same evaluation score. We investigate and improve tie-breaking strategies for cost-optimal search using $A^{*}$. We first experimentally analyze the performance of common tie-breaking strategies that break ties according to the heuristic value of the nodes. We find that the tie-breaking strategy has a significant impact on search algorithm performance when there are 0-cost operators that induce large plateau regions in the search space. Based on this, we develop two new classes of tie-breaking strategies. We first propose a depth diversification strategy which breaks ties according to the distance from the entrance to the plateau, and then show that this new strategy significantly outperforms standard strategies on domains with 0 -cost actions. Next, we propose a new framework for interpreting $\mathrm{A}^{*}$ search as a series of satisficing searches within plateaus consisting of nodes with the same f-cost. Based on this framework, we investigate a second, new class of tie-breaking strategy, a multi-heuristic tie-breaking strategy which embeds inadmissible, distance-to-go variations of various heuristics within an admissible search. This is shown to further improve the performance in combination with the depth metric.
\end{abstract}

\section{Introduction}

In this paper, we investigate tie-breaking strategies for cost-optimal $A^{*} . A^{*}$ is a standard search algorithm for finding an optimal cost path from an initial state $s$ to some goal state $g \in G$ in a search space represented as a graph (Hart, Nilsson, \& Raphael, 1968). It expands the nodes in best-first order of $f(n)$ up to $f^{*}$, where $f(n)$ is a lower bound of the cost of the shortest path that contains a node $n$ and $f^{*}$ is the cost of the optimal path. In many combinatorial search problems, the size of the last layer $f(n)=f^{*}$ of the search, called a final plateau, accounts for a significant fraction of the effective search space of $A^{*}$. Figure $1.1(\mathrm{p}, 68)$ compares the number of states in this final plateau with $f(n)=f^{*}$ (y-axis) vs. $f(n) \leq f^{*}$ (x-axis) for 1104 problem instances from the International Planning Competition (IPC1998-2011). For many instances, a large fraction of the nodes in the effective search space have $f(n)=f^{*}$ : The points are located very close to the diagonal line $(x=y)$, indicating that almost all states with $f(n) \leq f^{*}$ have cost $f^{*}$.

Figure 1.2 depicts this phenomenon conceptually. On the left, we show one natural view of the search space that considers the space searched by $A^{*}$ as a large number of closed nodes with $f<f^{*}$, surrounded by a thin layer of final plateau $f(n)=f^{*}$. This intuitive view accurately reflects the search spaces of some real-world problems such as 2D pathfinding on an explicit graph. It has also served as a model for algorithms such as Frontier Search (Korf, 1999; Korf \& Zhang, 2000), which tries to reduce the memory requirement by discarding the information associated with states with 
$f<f^{*}$, an effective strategy when the number of such states accounts for a large fraction of the memory usage.

However, for many other classes of combinatorial search problems, e.g., the IPC Planning Competition Benchmarks, the figure on the right is a more accurate depiction - here, the search space has a large plateau for $f=f^{*}$. In fact, Iterative Deepening approaches (Korf, 1985) assume this type of search space where this final frontier is quite large and the overhead of re-evaluating $f<f^{*}$ is limited. Classical planning problems in the IPC benchmark set are clearly the instances of such combinatorial search problems.



* openstacks-opt11

- parcprinter-opt1

- parking-opt11 pathways pegsol-opt11

$\triangle$ pipesworld-notankage

- pipesworld-tankage

$\checkmark$ psr-small

- rovers

$\diamond$ scanalyzer-opt11

- sokoban-opt11

+ storage

$\times$ tidybot-opt11

* tpp

- transport-opt11

- visitall-opt11

- woodworking-opt11

Figure 1.1: The number of nodes with $f=f^{*}$ (y-axis) compared to the total number of nodes in the search space (x-axis) with $f \leq f^{*}$ on 1104 IPC benchmark problems. This experiment uses a modified Fast Downward with LMcut which continues the search within the current $f$ after any cost-optimal solution is found. This effectively generates all nodes with cost $f^{*}$.

For the majority of such IPC problem domains where the last layer $\left(f(n)=f^{*}\right)$ accounts for a significant fraction of the effective search space, a tie-breaking strategy, which determines which node to expand among nodes with the same $f$-cost, can have a significant impact on the performance of $A^{*}$. It is widely believed that among nodes with the same $f$-cost, ties should be broken according to $h(n)$, i.e., nodes with smaller $h$-values should be expanded first. While this is a useful rule of thumb in many domains, it turns out that tie-breaking requires more careful consideration, particularly for problems where most or all of the nodes in the last layer have the same $h$-value.

We empirically evaluate the existing, commonly used, standard tie-breaking strategies for $A^{*}$ (Section 3). We show that:

1. In the experiments on IPC domains, A Last-In-First-Out (lifo) criterion tends to be more efficient than a First-In-First-Out (fifo) criterion. 


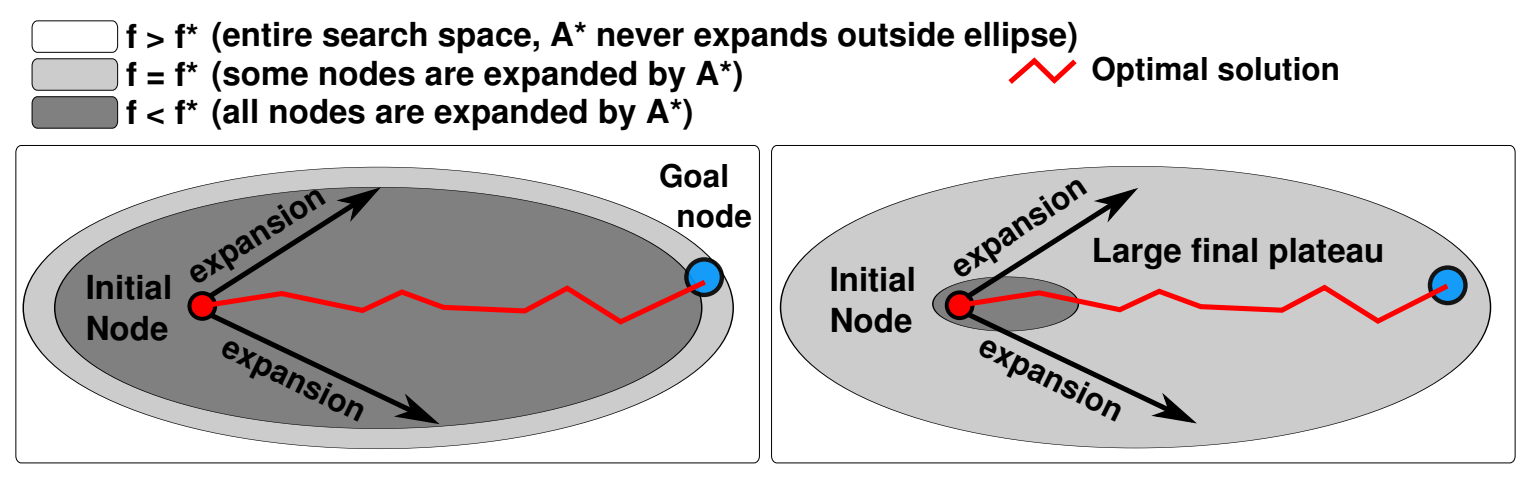

Figure 1.2: (Left) One possible class of search space which is dominated by the states with cost $f<f^{*}$. (Right) This paper focuses on another class of search space, where the plateau containing the cost-optimal goals $\left(f=f^{*}\right)$ is large, and it even accounts for most of the search effort required by $A^{*}$.

2. Tie-breaking according to the heuristic value $h$, which is frequently mentioned in the heuristic search literature, has little impact on the performance as long as lifo default criterion is used - in other words, a lifo tie-breaking policy is sufficient for most IPC domains.

3. There are significant performance differences among tie-breaking strategies when domains include 0 -cost actions. This is true even when $h$-based tie-breaking is used.

While there are currently relatively few standard benchmark domains with 0 -cost actions, we argue that 0 -cost actions naturally occur in practical cost-minimization problems. 0-cost actions induce $g$-value plateaus which are known to significantly increase difficulty of search (Benton, Talamadupula, Eyerich, Mattmüller, \& Kambhampati, 2010). Also, according to a parameterized complexity analysis, problem instances containing 0-cost actions are harder than the instances with strictly positive action costs (Aghighi \& Bäckström, 2015). Therefore, we introduce a new set of benchmarks called Zerocost domains (Section 4), which are a set of domains based on standard IPC domains for which only the most important actions directly related to resource usage incur the nonzero costs. We compare these domains with the original IPC domains from which they are derived, and empirically show that Zerocost domains have a different search space structure and pose different, practical difficulties. Hereafter, we use " 0 -cost" as a general adjective for actions and search edges, while "Zerocost" refers to this specific set of benchmark domains we introduce in this paper.

In order to solve such problems more efficiently, we propose and evaluate depth diversification, a new tie-breaking method based on the notion of a node's depth within a plateau, which corresponds to the number of steps from the "entrance" to the plateau (Section 5, Section 6). Our new depthbased diversification strategy significantly improves upon the standard tie-breaking strategies.

We then propose a new framework which considers cost-optimal search using $A^{*}$ as a series of satisficing searches on each plateau. This allows the problem of tie-breaking to be reduced to satisficing search within a plateau (Section 77. Based on this insight, we then investigate an admissible tie-breaking strategy which uses the distance-to-go estimate, a heuristic function which treats every action to have the unit costs (Section 8). Although distance-to-go estimates are inadmissible, it does 
compromise the admissibility of $A^{*}$ as long as it is used only for tie-breaking. We empirically show that:

1. Tie-breaking using distance-to-go variations of LMcut, M\&S and FF heuristics, with LMcut or M\&S as a primary heuristics for computing $f$ value (for maintaining admissibility), significantly improves the standard tie-breaking strategies.

2. Combining depth-based diversification with distance-to-go heuristics further improves the performance.

This paper significantly extends an earlier conference paper (Asai \& Fukunaga, 2016), which experimentally evaluated standard tie-breaking strategies, identified the significant effect of tiebreaking strategies in domains with zero-cost edges, and proposed randomized depth-based diversification. In this paper we present an expanded analysis of domains with zero-cost edges, a new, deterministic, depth-based diversification strategy, and an expanded empirical and theoretical analysis of depth-based diversification. We also introduce the framework for treating $A^{*}$ as a sequence of satisficing searches on a set of $f$-cost plateaus, and propose tie-breaking strategies which incorporates distance-to-go estimates.

\section{Preliminaries and Definitions}

We first define some notation and terminology used throughout the rest of the paper. $h(n)$ denotes the estimate of the cost from the current node $n$ to the nearest goal node. $g(n)$ is the current shortest known path cost from the initial node to the current node. $f(n)=g(n)+h(n)$ is the estimate of the cost of a path to a goal containing the current node. We omit the argument $(n)$ unless necessary.

Below, we first present a general Best First Search (BFS) algorithm template which includes $A^{*}$, Dijkstra's algorithm (1959), Greedy Best-First Search (GBFS). It uses two sets, OPEN and CLOSED, where unexpanded nodes are stored in OPEN and expanded nodes are stored in CLOSED. Three operations, $\operatorname{pop}(S), \operatorname{push}(n, S)$ and remove $(n, S)$, are assumed for a node $n$ and a set $S$. $\operatorname{pop}(S)$ operation tries to select a single node from $S, \operatorname{push}(n, S)$ stores the node $n$ into $S$ and remove $(n, S)$ removes $n$ from $S$ if $n$ is already stored.

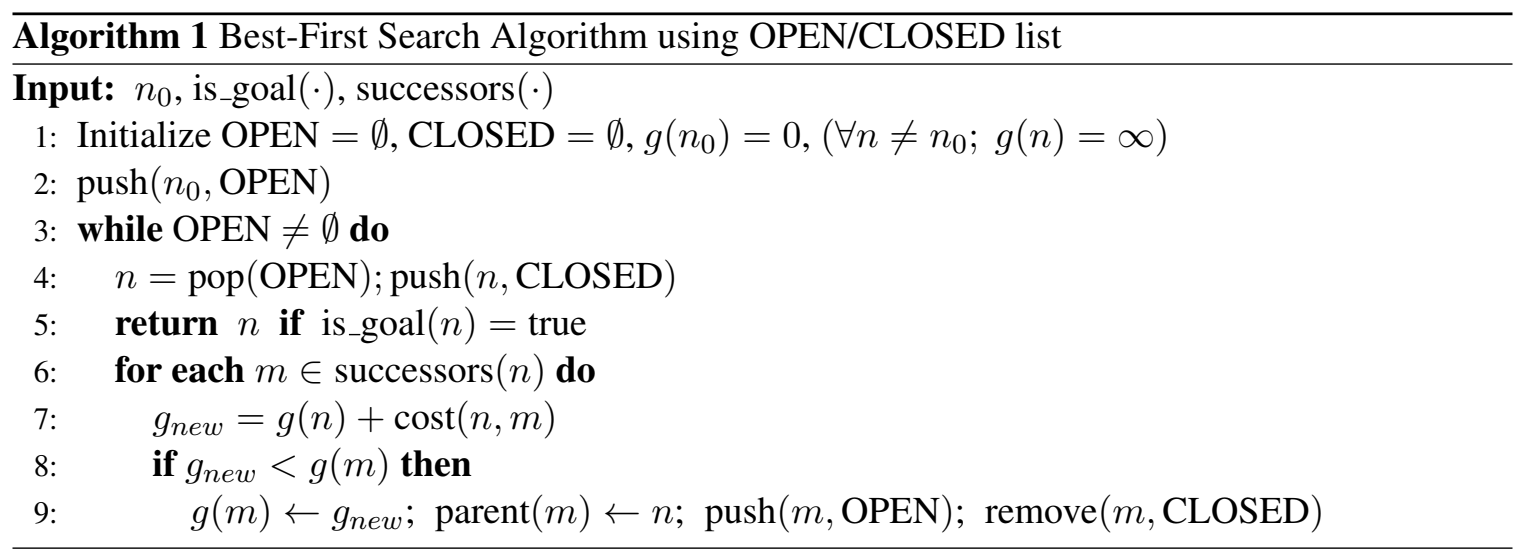

OPEN is sorted according to a sorting strategy and the node selected by $\operatorname{pop}(S)$ always returns the best node according to the strategy. Each sorting strategy is denoted as a vector of several sorting 
criteria, such as $\left[\right.$ criterion $_{1}$, criterion $_{2}, \ldots$, criterion $\left._{k}\right]$, which defines a lexicographic ordering, i.e., from the OPEN list, first, select a set of nodes using criterion ${ }_{1}$, and if there are still multiple nodes remaining in the set, then break ties using criterion ${ }_{2}$ and so on, until a single node is selected. The first-level sorting criterion of a strategy is criterion ${ }_{1}$, the second-level sorting criterion is criterion ${ }_{2}$, and so on 1

Using this notation, $A^{*}$ without any tie-breaking strategy can be denoted as a BFS with $[f]$ and $A^{*}$ which breaks ties according to $h$ value is denoted as $[f, h]$. Unless stated otherwise, we assume the nodes are sorted in the increasing order of the key value and a BFS always selects the smallest key value.

However, a sorting strategy may only provide a partial ordering, i.e., the sorting strategy may fail to select a single node because some nodes may share the same sorting keys. For such cases, a BFS algorithm must decide which node to expand by applying some default tie-breaking criterion criterion $_{k}$ which is guaranteed to return a single node, such as fifo (oldest node first: first-in-firstout), lifo (most recently inserted first: last-in-first-out) or ro (random ordering). For example, $A^{*}$ using $h$ tie-breaking and fifo default tie-breaking is denoted as $[f, h, f i f o]$. By definition, there is only 1 node which satisfies the default criterion, so strategies with a default criterion guarantee a total ordering among all nodes and are able to select a single node from the set of nodes. When the default criterion is irrelevant to the discussion, we either use a wildcard "*”, e.g. $[f, h, *]$, or sometimes omit it altogether for brevity.

Given a search algorithm with a sorting strategy, a plateau (criterion...) is a set of nodes in OPEN whose elements share the same sort keys according to non-default sorting criteria and are therefore indistinguishable. In the case of $A^{*}$ using tie-breaking with $h$ (sorting strategy $[f, h, *]$ ), the plateaus are denoted as plateau $(f, h)$, the set of nodes with the same $f$ cost and the same $h$ cost. We can also refer to a specific plateau with $f=f_{p}$ and $h=h_{p}$ by plateau $\left(f_{p}, h_{p}\right)$.

An entrance to a plateau (criterion...) $=P$ is a node $n \in P$, whose current parent is not in $P$. The final plateau is the plateau containing the solution found by the search algorithm. In $A^{*}$ using admissible heuristics, the final plateau is plateau $\left(f^{*}\right)$ (without tie-breaking), or plateau $\left(f^{*}, 0\right)$ (with $h$-based tie-breaking).

\subsection{Tie-Breaking Strategies for $A^{*}$}

$A^{*}$ is a standard search algorithm for finding an optimal cost path on a graph. On a finite graph, $A^{*}$ is complete regardless of the tiebreaking strategy (Hart et al., 1968).

It can be defined as a subclass of BFS which uses $f$-value as the first sorting criterion and returns a cost-optimal solution when $h$ is admissible, i.e., when $\forall n ; h(n) \leq h^{*}(n)$, where $h^{*}(n)$ is the optimal distance from $n$ to the nearest goal. The best-first order of the expansion is the key to guaranteeing solution optimality. The first solution found by the algorithm is guaranteed to have the optimal cost $f=f^{*}$ because all nodes with $f(n)<k$ are already expanded when it starts expanding the nodes with $f(n)=k$. Thus, the effective search space of $A^{*}$ is the set of nodes with $f(n) \leq f^{*}$ : $A^{*}$ expands all nodes with $f(n)<f^{*}$, then expands some of the nodes with $f(n)=f^{*}$, and never expands the nodes with $f(n)>f^{*}$.

If there are multiple nodes with the same $f$-cost, $A^{*}$ must implement some tie-breaking strategy (either explicitly or implicitly) which selects from among these nodes. The early literature on heuristic search seems to have been mostly agnostic regarding tie-breaking. The original $A^{*}$ paper,

1. This notation corresponds to the command line option format of Fast Downward (Helmert, 2006). 
as well as Nilsson's subsequent textbook states: "Select the open node $n$ whose value $f$ is smallest. Resolve ties arbitrarily, but always in favor of any [goal node]" (Hart et al., 1968, p. 102 Step 2; Nilsson, 1971, p. 69). Pearl's textbook on heuristic search specifies that best-first search should "break ties arbitrarily" (Pearl, 1984, p. 48, Step 3), and does not specifically mention tie-breaking for $A^{*}$. To the best of our knowledge, the first explicit mention of a tie-breaking strategy that considers node generation order is by Korf in his analysis of IDA*: "If $A^{*}$ employs the tie-breaking rule of 'most-recently generated', it must also expand the same nodes [as IDA*]", i.e., a lifo ordering.

In recent years, tie-breaking according to $h$-values has become "folklore" in the search community. Hansen and Zhou state that "[i]t is well-known that $A^{*}$ achieves best performance when it breaks ties in favor of nodes with least h-cost" (Hansen $\&$ Zhou, 2007). Holte writes " $A^{*}$ breaks ties in favor of larger $g$-values, as is most often done" (Holte, 2010). Note that preferring large $g$ is equivalent to preferring smaller $h$, since $f=g+h$. Felner et al. also assume "ties are broken in favor of low h-values" in describing Bidirectional Pathmax for $A^{*}$ (2011). In their detailed survey/tutorial on efficient $A^{*}$ implementations, Burns et al. (2012) also break ties "preferring high $g$ " (equivalent to low $h$ ). Thus, tie-breaking according to $h$-values appears to be ubiquitous in practice. However, to our knowledge, an in-depth experimental analysis of tie-breaking strategies for $A^{*}$ is lacking in the literature.

Although the standard practice of tie-breaking according to $h$ might be sufficient in some domains, further levels of tie-breaking (explicit or implicit) are required if multiple nodes have the same $f$ as well as the same $h$ values. To date, the effect of such default tie-breaking has not been investigated in depth. For example, although the survey of efficient $A^{*}$ implementation techniques by Burns et al. did not explicitly mention the default tie-breaking (2012), their library code uses lifo default tie-breaking (Burns, 2012). It first breaks ties according to $h$, and then breaks remaining ties according to a lifo criterion (most recently generated nodes first), i.e., $[f, h, l i f o]$. Although not documented, their choice of a lifo 2nd-level tie-breaking criterion appears to be a natural consequence of the fact it can be trivially and efficiently implemented in their two-level bucket (vector) implementation of OPEN. In contrast, the current implementation of the State-of-the-Art $A^{*}$ based planner Fast Downward (Helmert, 2006), as well as the work by Röger and Helmert (2010) uses a $[f, h, f i f o]$ tie-breaking strategy. Although we could not find a published explanation, this choice is most likely due to their use of alternating OPEN lists, in which case the fifo second-level criterion serves to provide a limited form of fairness.

\section{Analysis of Standard Strategies}

We first we evaluated standard tie-breaking strategies for domain-independent cost-optimal classical planning and analyze their performance differences. In our experiments, all planners are based on Fast Downward, and all experiments are run with a 5-minute, 4GB memory limit for the search binary (FD translation/preprocessing times are not included in the 5-minute limit). All experiments were conducted on Xeon E5410@2.33GHz CPUs. For the randomized configurations, we took the average of 10 runs. We used two State-of-the-Art heuristic functions LMcut (Helmert \& Domshlak, 2009) and M\&S (Helmert, Haslum, Hoffmann, \& Nissim, 2014) as the primary heuristic functions used for calculating $f$ and $h$. For M\&S, we used the bisimulation-based shrink strategy, DFP merge strategy, and exact label reduction. These basic experimental configurations are shared in all performance evaluation experiments throughout this paper. 
We used 1104 instances from 35 standard IPC benchmark domains: airport (50 instances), barman-opt11(20), blocks(35), cybersec(19), depot(22), driverlog(20), elevators-opt11(20), floortile-opt11(20), freecell(80), grid(5), gripper(20), hanoi(30), logistics00(28), miconic(150), mprime(35), mystery(30), nomystery-opt11(20), openstacks-opt11(20), parcprinter-opt11(20), parking-opt11(20), pathways(30), pegsol-opt11(20), pipesworld-notankage(50), pipesworld-tankage(50), psr-small(50), rovers(40), scanalyzer-opt11(20), sokoban-opt11(20), storage(30), tidybot-opt11(20), tpp(30), transport-opt11(20), visitall-opt11(20), woodworking-opt11(20), zenotravel(20).

\subsection{Is $h$-Based Tie-Breaking Necessary?}

As noted in Section 2.1, the current standard practice is to use a tie-breaking criterion which uses the $h$-value of the nodes. However, to our knowledge, the need for $h$-based tie-breaking has not been previously empirically investigated.

In Table 3.1, we show the summary results for $[f$, fifo $]$ and $[f$, lifo $]$, the $A^{*}$ variants which rely on fifo or lifo default tie-breaking only, as well as the standard $[f, h, f i f o]$ and $[f, h, l i f o]$ strategies. (Detailed results are in Table A.1 and Table A.2 in the Appendix.) $[f$, lifo], which simply breaks ties among nodes with the same $f$-cost by expanding the most recently generated nodes first (Korf, 1985), clearly dominates $[f$, fifo $]$. Interestingly, the performance of the $[f$, lifo $]$ strategy is comparable to $[f, h$, lifo $]$ and $[f, h, f i f o]$. This may be surprising, considering the ubiquity of $h$-based tie-breaking in the search and planning communities.

This is explained by the fact that lifo behaves somewhat similarly to $h$-based tie-breaking. lifo expands the most recently generated node $n$. For any child $n^{\prime}$, if the heuristic function is admissible and $f\left(n^{\prime}\right)=f(n)$, there are only 2 possibilities : (1) $g\left(n^{\prime}\right)>g(n)$ and $h\left(n^{\prime}\right)<h(n)$, or (2) $g\left(n^{\prime}\right)=g(n)$ and $h\left(n^{\prime}\right)=h(n)$. Thus, as lifo expands nodes in a "depth-first" manner, the nodes that continue to be expanded in plateau $(f)$ by lifo usually have non-increasing $h$-values, much like in $h$-based tie-breaking which always searches toward the least $h$ cost. Thus, although the expansion order of $[f$, lifo $]$ is not exactly the same as that of $h$-based tie-breaking strategies, they perform similarly.

\subsection{Do Default Strategies Make a Difference?}

Next, we compared two commonly used tie-breaking strategies, $[f, h, f i f o],[f, h, l i f o]$, which first break ties according to $h$, and then apply fifo or lifo default tie-breaking, respectively. Summary results for LMcut and M\&S are shown in Table 3.1, and the detailed results are in Table A.1 and Table A.2 (Section A Appendix). Differences in coverage are observed in several domains and $[f, h$, lifo $]$ outperforms $[f, h, f i f o]$ overall. Thus, the choice of default criterion seems to have a modest but measurable impact when the first tie-breaking criterion is $h$.

We also conducted experiments using ro (Random Order) default tie-breaking because it is another trivial way to break ties. We ran the experiments 10 times with the different random seeds, then took the average and the standard deviation of the coverages. The performance of ro is comparable to fifo default tie-breaking regardless of the primary heuristics, or the presence of $h$-based tie-breaking. 


\begin{tabular}{|l|cc|} 
Sorting Criteria & $\begin{array}{c}\text { IPC(1104) } \\
\text { LMcut }\end{array}$ & $\begin{array}{c}\text { IPC(1104) } \\
\text { M\&S }\end{array}$ \\
{$[f$, fifo $]$} & 443 & 460 \\
{$[f$, lifo $]$} & 558 & 490 \\
{$[f, r o]$} & $448.9 \pm 1.3$ & $460.9 \pm 1.6$ \\
& & \\
{$[f, h$, fifo $]$} & 558 & 491 \\
{$[f, h$, lifo $]$} & $\mathbf{5 6 5}$ & $\mathbf{4 9 6}$ \\
{$[f, h$, ro $]$} & $558.9 \pm 2.1$ & $489.4 \pm 1.0$
\end{tabular} \mid

Table 3.1: Summary of coverage comparison (the number of instances solved in 5min, 4GB, LMcut heuristics) among the standard baseline tie-breaking algorithms (details in Table A.1 and Table A.2, leftmost 2 columns).

\subsection{Plateaus and Tie-Breaking}

Figure 3.1 provides a more fine-grained analysis by comparing the number of node evaluations (calls to the expensive LMcut heuristic function) on each instance by the $[f, h$, lifo $]$ and $[f, h, f i f o]$ strategies. The difference in the number of nodes evaluated can sometimes be larger than a factor of 10 (Openstacks, Cybersec domains). As noted in Section 2.1, the choice among default criteria has not been considered very important in the literature, as evidenced by the lack of explicit descriptions of the default tie-breaking criterion in recent papers. Our results suggest that 2 nd-level default tiebreaking can have a surprisingly large effect on the search performance.

The effect of the choice of 2nd-level default tie-breaking criteria (lifo vs. fifo) when the 1st-level tie-breaking criterion is $h$ tie-breaking is limited to each search plateau plateau $(f, h)$, the set of nodes which share the same $f$ value and $h$ value. Also, in admissible search, two $A^{*}$ implementations using different default tie-breaking criteria both expand the same set of nodes in the region where $f<f^{*}$. Furthermore, nodes with $h>0$ can not be goal nodes when $h$ is admissible. Therefore, the effect of default tie-breaking becomes most prominent in the final plateau, plateau $\left(f^{*}, 0\right)$.

Counterintuitively, the plateau $\left(f^{*}, 0\right)$ region can be large enough to cause a significant performance difference - in fact, this final plateau can even account for most of the search effort required by $A^{*}$. Figure 3.2 plots the size of the final plateau on 1104 IPC benchmark instances. The $y$-axis represents the number of nodes in the final plateau (plateau $\left(f^{*}, 0\right)$ ), and the $x$-axis represents the total number of nodes expanded so far. This figure suggests that in some domains such as Openstacks and Cybersec, the planner spends most of the runtime searching plateau $\left(f^{*}, 0\right)$ for a solution, even with the help of $h$ tie-breaking.

A natural question is: What makes these two domains (Openstacks and Cybersec) different from all other domains which have much smaller final plateaus?

\section{Domains with 0-Cost Actions}

Openstacks is a cost minimization domain introduced in IPC-2006, where the objective is to minimize the number of stacks used. One characteristic of Openstacks is the presence of many actions 

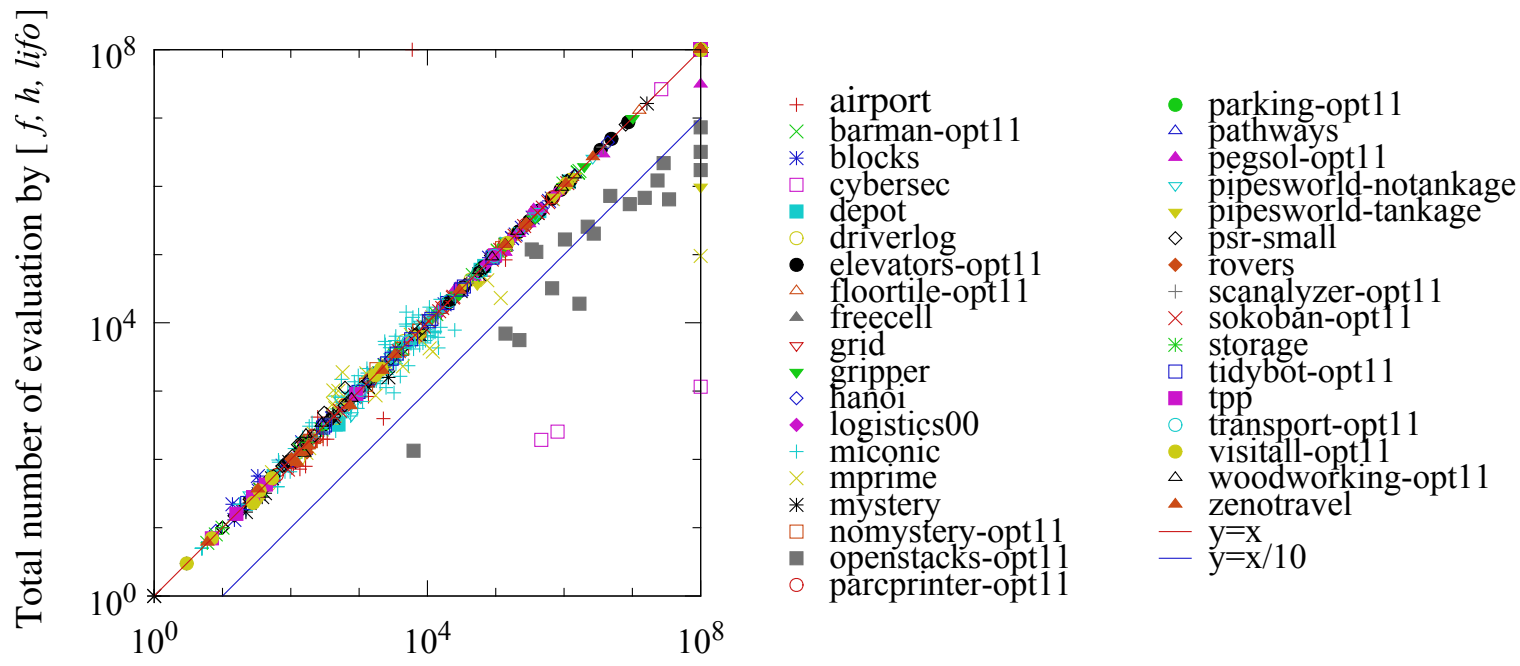

Total number of evaluation by $[f, h$, fifo $]$

Figure 3.1: The number of LMcut evaluations on various IPC planning benchmark domains, with standard fifo vs lifo default tie-breaking, both with $h$ tie-breaking. lifo evaluates less than $1 / 10$ of the nodes evaluated by fifo in Cybersec and Openstacks.
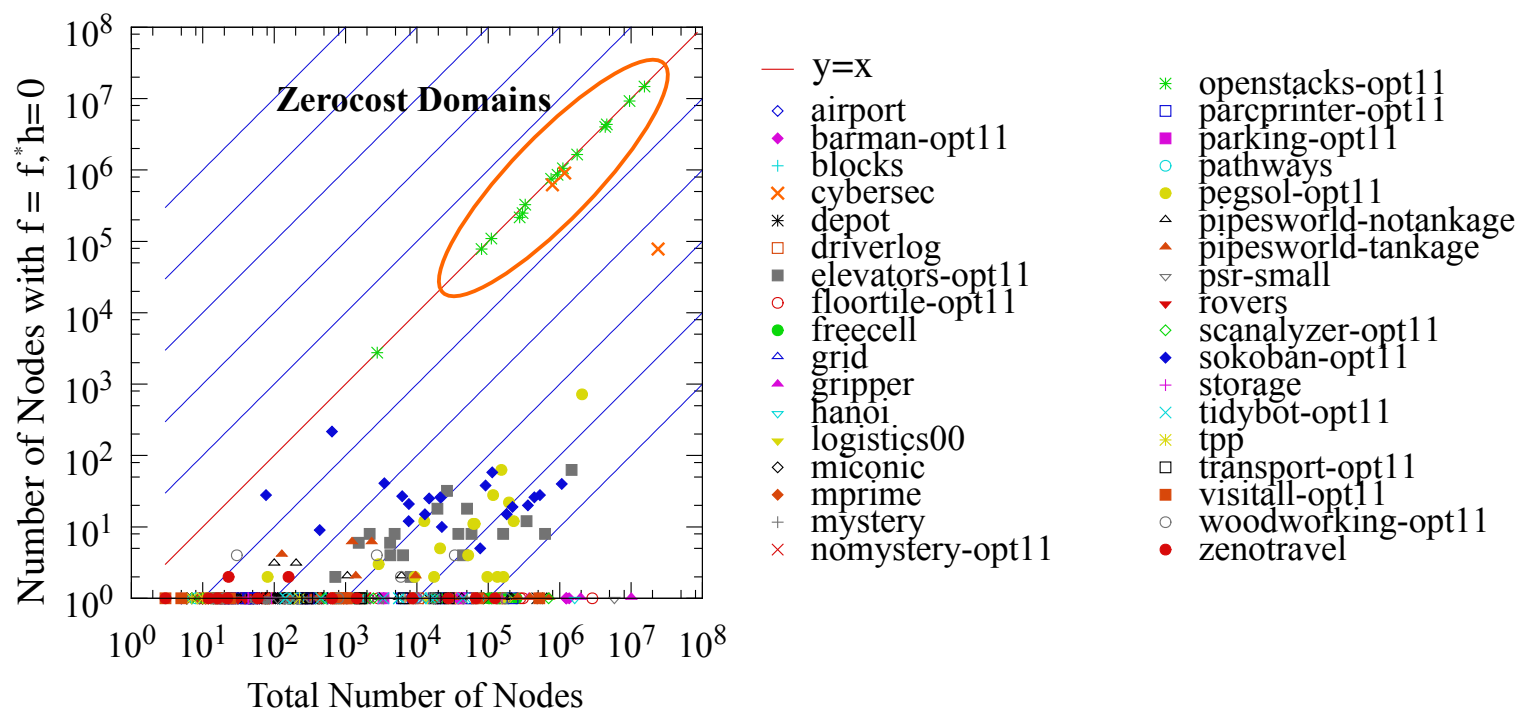

Figure 3.2: The number of nodes in plateau $\left(f^{*}, 0\right)$ (y-axis), which form the final plateau for sorting strategy $[f, h]$, compared to the total number of nodes in the search space with $f \leq$ $f^{*}$ (x-axis) on 1104 IPC benchmark problems. Note that Openstacks and Cybersec instances are near the $y=x$ line. These statistics are obtained by running a modified Fast Downward with LMcut which continues searching after the solution is found until all nodes with cost $f=f^{*}$ are expanded. 
which have zero cost because they do not increase the number of stacks. These 0 -cost actions create the problem depicted in Figure 4.1. Since 0-cost actions (edges) allow "free" transitions between many neighboring nodes, the number of neighboring nodes sharing the same $h$ also becomes quite large. This creates huge plateaus that share the same $h$-value, and the standard $h$-based tie-breaking criterion can not provide informative guidance for search within a plateau. Since the $g$-values of the nodes in these plateaus are all identical, these plateaus are an instance of $g$-value plateaus, which are known to increase the difficulty of search (Benton et al., 2010).

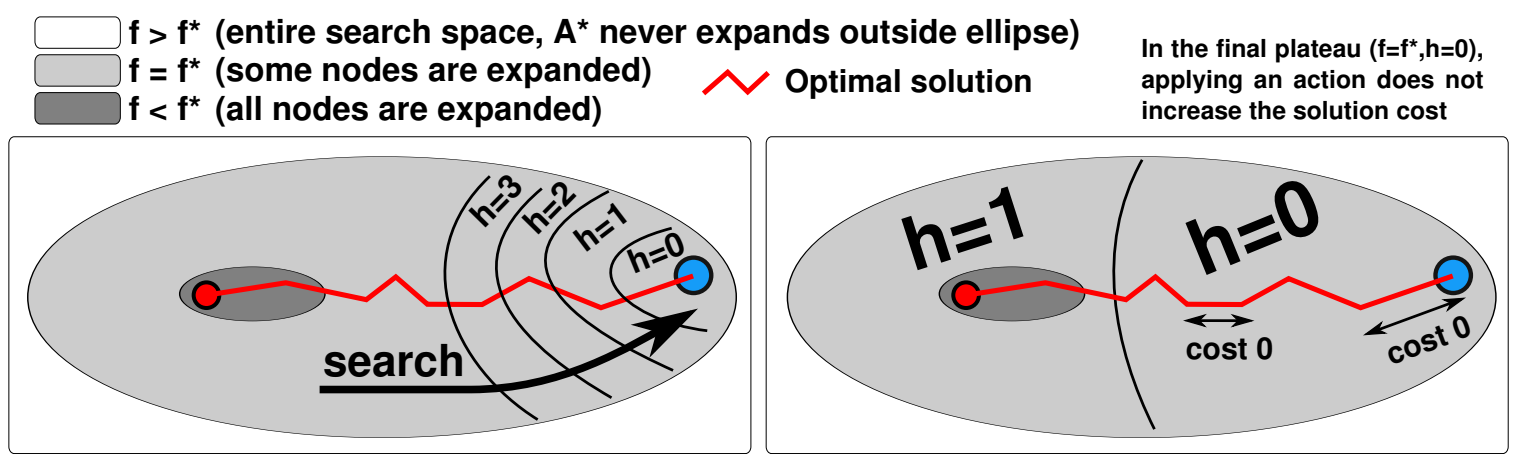

Figure 4.1: Search space of $A^{*}$ and its contour according to admissible heuristic $h$. (Right) In domains with only positive-cost actions, $h$-based tie-breaking provides meaningful guidance. (Left) In domains with 0-cost actions, applying an action may not increase the cost of the path and the region with $h=0$ could be quite large. With the same mechanism, other heuristic plateaus (e.g. $h=1$ ) also become larger. Thus, $h$-based tie-breaking fails to provide meaningful guidance in this space.

Although most traditional benchmark problems in the planning community and the combinatorial search community do not have 0-cost actions, we argue that such domains are of an important class of models for cost-minimization problems, i.e., assigning 0-costs makes sense from a practical, modeling perspective. For example, consider the driverlog domain, where the task is to move packages between locations using trucks. The IPC version of this domain assigns unit costs to all actions. Thus, cost-optimal planning on this domain seeks to minimize the number of steps in the plan. However, another natural objective function would be the one which minimizes the amount of fuel spent by driving the trucks, assigning cost 0 to all actions except drive-truck - we believe that for cost-optimal planning, this is at least as natural as the current IPC model of driverlog in which all actions are of unit cost.

Similarly, for many practical applications, a natural objective is to optimize the usage of one key consumable resource, e.g., fuel/energy minimization. In fact, two of the IPC domains, Openstacks and Cybersec, which were shown to be difficult for standard tie-breaking methods in the previous section, both contain many 0 -cost actions and are based on industrial applications: Openstacks models production planning (Fink \& Voss, 1999) and Cybersec models Behavioral Adversary Modeling System (Boddy, Gohde, Haigh, \& Harp, 2005, minimizing decryption, data transfer, etc.).

Therefore, in this paper, we modified various standard domains into cost minimization domains with many 0-cost actions. Specifically, each of our "Zerocost domains" is a standard domain which 
has been modified so that all action schema are assigned cost 0 except for a few (usually one) action schema which consumes some key resource. The suffixes in the names of these domains indicate the actions with non-zero costs, e.g., logistics-fuel is a modified logistics domain where only actions which consume fuel have non-zero cost. Most of the transportation-type domains are modified to optimize energy usage (logistics-fuel, elevator-up etc.), and assembly-type domains are modified to minimize resource usage (Woodworking-cut minimizes wood usage, etc.). When no action makes sense from the practical point of view, we chose an action schema arbitrarily (e.g. mprime-succumb). We did not include domains which have only a single action schema, or which already had many 0 -cost actions.

The new set of 28 Zerocost domains are: airport-fuel (20 instances), blocks-stack (20), depotfuel (22), driverlog-fuel (20), elevators-up (20), floortile-ink (20), freecell-move (20), grid-fuel (5), gripper-move (20), hiking-fuel (20), logistics00-fuel (28), miconic-up (30), mprime-succumb (35), mystery-feast (20), nomystery-fuel (20), parking-movecc (20), pathways-fuel (30), pipesnt-pushstart (20), pipesworld-pushend (20), psr-small-open (20), rovers-fuel (40), scanalyzer-analyze (20), sokoban-pushgoal (20), storage-lift (20), tidybot-motion (20), tpp-fuel (30), woodworking-cut (20), zenotravel-fuel (20).

While the action costs in the PDDL domain definitions are modified, we did not modify the PDDL problem definitions. Although some domains (specifically, blocks, freecell, pipesworld-notankage, miconic) have fewer instances than the original domain does, their problem definitions are the evenly sampled subset of the original set of instances. For example, the original miconic domain has 150 instances, while our version has 30 instances. These 30 instances are selected evenly from the original set of instances, by picking instances $\mathrm{p} 05, \mathrm{p} 10, \ldots \mathrm{p} 150$. The reason for reducing the number of instances is to avoid the problem of the overall coverage sums being skewed by the domains with a larger number of instances. Thus, we did not modify the problem definitions at all, and only modified the action costs in the domain definitions.

\subsection{Difference in Problem Characteristics between IPC and Zerocost Domains}

Domains containing 0-cost operators are known to be difficult for traditional planners (Thayer \& Ruml, 2009; Cushing, Benton, \& Kambhampati, 2010; Wilt \& Ruml, 2011; Thayer \& Ruml, 2011; Richter, Westphal, \& Helmert, 2011). Cushing et al. (2010) and Wilt and Ruml (2011) noted that a large ratio between maximum and minimum operator costs can pose a challenge to existing planners. They both addressed this using plan-length heuristics instead of plan-cost heuristics, which sacrifice the optimality of the solution. In contrast, we investigate methods for handling 0 -cost operators within the framework of admissible search. In Section 8 , we show how plan length heuristics can be incorporated into admissible search. In a parameterized complexity analysis of planning domains, Aghighi and Bäckström $(2015,2016)$ showed that domains with 0-cost operators comprise a complexity class that is harder (para-NP-hard) than the domains with strictly positive-cost operators (W[2] complete), indicating the inherent difficulty of optimally solving planning problems with 0 -cost actions.

Therefore we experimentally evaluate whether our new set of Zerocost benchmarks based on standard IPC domains pose a new challenge for standard tie-breaking strategies. Results using the LMcut heuristic are shown in Table 4.1. In each table, the left-hand side shows the results in the original domains and the right-hand side shows the results for the corresponding Zerocost domains. 
We observed a significant performance difference between the original IPC domains and the Zerocost domains. The coverage in Zerocost domains was lower in 11 domains while more instances were solved in 5 domains. The coverage increase in some domains is not surprising, considering that 0 -cost actions also make some suboptimal paths into cost-optimal paths. However, the coverage decreased overall, confirming the difficulty of these domains.

Figure 4.2 plots the size of the final plateau of the Zerocost instances, with LMcut heuristics and $h$ tie-breaking. In this plot, each point shows the total number of nodes in plateau $\left(f^{*}, 0\right)$ vs the total number of nodes with $f \leq f^{*}$. Compared to Figure 3.2, most Zerocost instances have larger plateaus even with the help of $h$ tie-breaking. Thus, in these cost-minimization problems, the search strategy within plateaus, i.e., tie-breaking, becomes even more critical in determining search performance.

$\operatorname{depot}(22)$

driverlog $(20)$

elevators-opt11(20)

floortile-opt11(20)

$\operatorname{grid}(5)$

gripper(20)

logistics00(28)

mprime(35)

nomystery-opt11(20)

parking-opt11(20)

pathways(30)

rovers(40)

scanalyzer-opt11(20)

sokoban-opt11(20)

storage (30)

tidybot-opt11(20)

$\operatorname{tpp}(30)$

woodworking-opt11(20)

zenotravel(20)

\begin{tabular}{c|cc}
{$[f, h, f i f o]$} & {$[f, h$, fifo $]$} & (difference) \\
6 & 6 & \\
13 & 8 & $(-5)$ \\
15 & 7 & $(-8)$ \\
6 & 8 & $(+2)$ \\
1 & 1 & \\
6 & 7 & $(+1)$ \\
20 & 16 & $(-4)$ \\
21 & 15 & $(-6)$ \\
14 & 10 & $(-4)$ \\
1 & 0 & $(-1)$ \\
5 & 5 & \\
7 & 8 & $(+1)$ \\
10 & 9 & $(-1)$ \\
19 & 18 & $(-1)$ \\
14 & 4 & $(-10)$ \\
12 & 16 & $(+4)$ \\
6 & 8 & $(+2)$ \\
10 & 5 & $(-5)$ \\
11 & 7 & $(-4)$
\end{tabular}

depot-fuel(22)

driverlog-fuel(20)

elevators-up(20)

floortile-ink(20)

grid-fuel(5)

gripper-move(20)

logistics00-fuel(28)

mprime-succumb(35)

nomystery-fuel(20)

parking-movecc(20)

pathways-fuel(30)

rovers-fuel(40)

scanalyzer-analyze (20)

sokoban-pushgoal(20)

storage-lift(20)

tidybot-motion(20)

tpp-fuel(30)

woodworking-cut(20)

zenotravel-fuel(20)

Table 4.1: Assessment of the relative difficulty of Zerocost domains vs. their corresponding standard domains, for the standard $[f, h, f f o]$ strategy. Coverage comparison (the number of instances solved) between the original IPC domains and the modified Zerocost domains are shown, using the same planner configuration and experimental setting (5min, 4GB, LMcut heuristics). This table does not include domains where the total number of instances in the Zerocost domain and the original domain differ.

Note that the difficulty posed by these domains sometimes cannot be tackled by improving the heuristic estimates, or reducing the underestimation of an admissible heuristic function. Due to the existence of 0-cost edges, some non-goal neighbors of a goal node have $h^{*}=0$. For those 


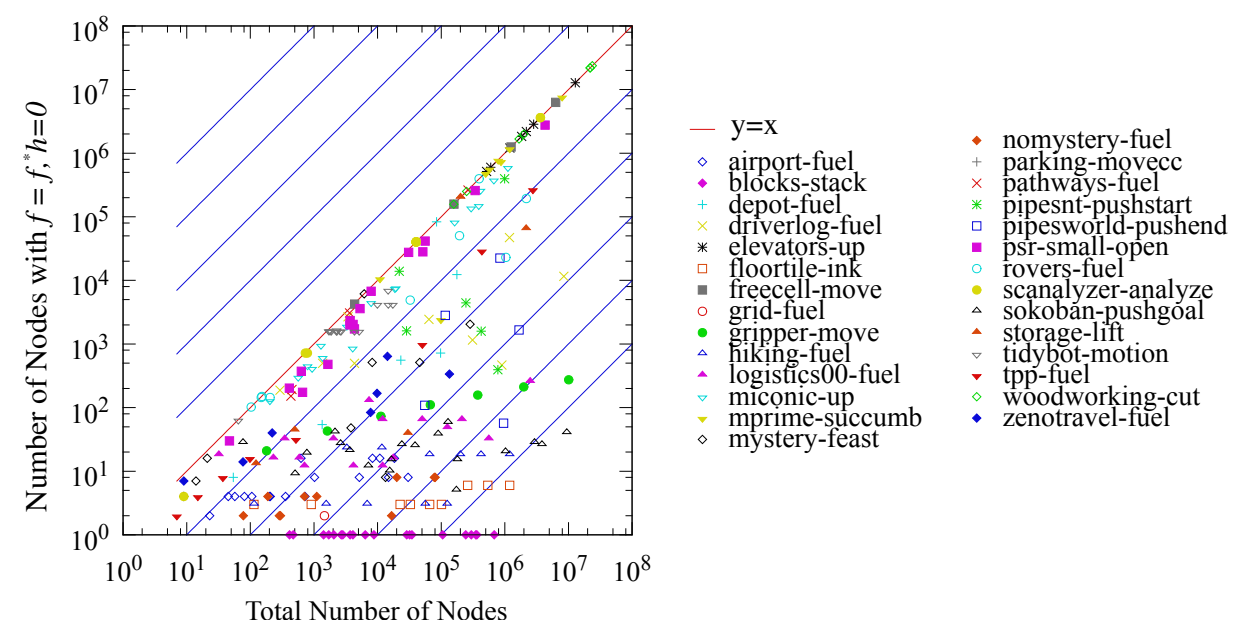

Figure 4.2: The number of nodes in plateau $\left(f^{*}, 0\right)$ (y-axis), which form the final plateau under $h$ based tie-breaking, compared to the total number of nodes in the search space (x-axis) with $f \leq f^{*}$ on 620 instances in our Zerocost domains. The final plateaus tends to account for a larger portion of the entire search space compared to Figure 3.2. These statistics are obtained by running a modified Fast Downward with LMcut which continues searching after the solution is found until expanding all nodes with cost $f=f^{*}$.

nodes, there is clearly no room for improving the heuristic estimate; Any positive value causes the heuristics to be inadmissible.

One approach to improving the search performance in such plateaus produced by 0 -cost edges is to perform an efficient knowledge-free search within plateau; It may reuse the effort that is already spent to guide the search but without requiring additional effort to compute multiple heuristics. In the next section, we propose and evaluate an implementation of such a technique. It turns out that introducing a notion of depth within a plateau can have a significant impact on the performance of knowledge-free search, and can also provide a good understanding of the behavior of standard tie-breaking strategies.

\section{Depth-Based Tie-Breaking for $\mathbf{A}^{*}$}

As shown in the previous section, the search spaces of Zerocost domains have many 0-cost edges, resulting in a large final plateau (plateau $\left(f^{*}, 0\right)$ ). In a final plateau, all nodes have $h=0$, so $h$ based tie-breaking cannot provide useful guidance toward a goal. Thus, we need a new metric for discriminating among nodes in the plateau so that the search algorithm can make progress in the plateau.

We define the depth of a node as an integer representing the distance (number of steps) from the entrance of the plateau. An entrance of the plateau is the first node which encountered the plateau along the path from the initial node. These notions are depicted in Figure 5.1 (subfigure 1).

The depth $d(n)$ of a node $n$ is 0 when $n$ and the parent node $m$ are in the different plateaus, and $d(n)=d(m)+1$ when they are on the same plateau. As defined in Section 2 , if two nodes are on the same plateau, they share the same key values for the sorting strategy. For example, when 

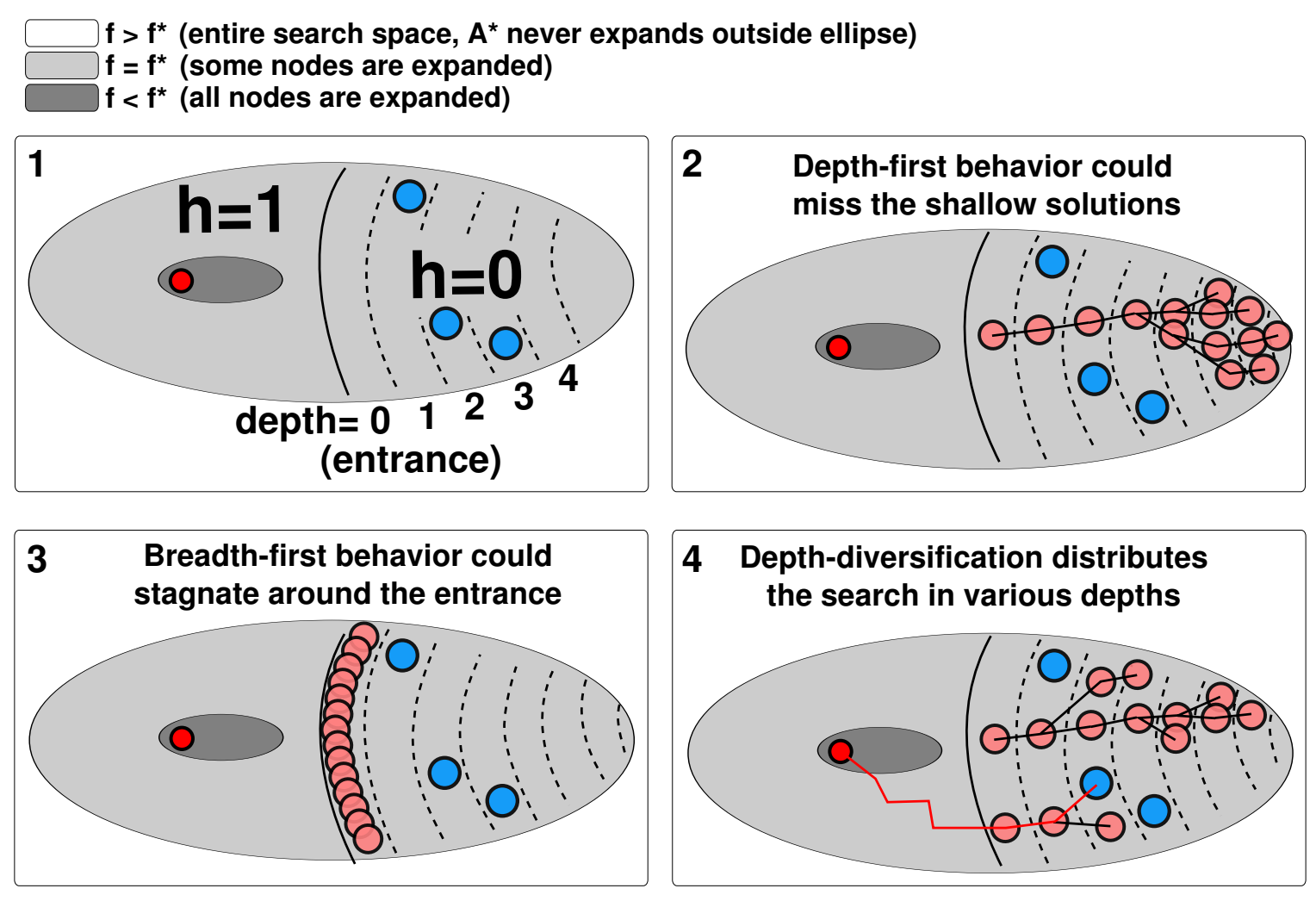

Figure 5.1: (Subfigure 1) The nodes in a plateau are divided into several layers, and each layer has a corresponding depth. Since all nodes have $f=f^{*}$, depth does not affect optimality, so all goals in the final plateau are cost-optimal, regardless of whether they are in shallow/deep regions. (Subfigure 2) lifo tie-breaking strategy results in depth-first behavior in a plateau, which could miss solutions if they are concentrated near the entrance. (Subfigure 3) fifo tie-breaking strategy results in breadth-first behavior in a plateau, which could fail to reach solutions in deeper layers within the time limit. (Subfigure 4) Depth-based diversification allows $A^{*}$ to search the plateau space in a less biased manner. This balances exploration and exploitation, avoiding the problems with both lifo (depth-first) and fifo (breadth-first) behavior. 
the strategy is $[f, h, *]$, it means plateau $(f(n), h(n))=$ plateau $(f(m), h(m))$, therefore $f(n)=$ $f(m) \wedge h(n)=h(m)$.

The traditional lifo and fifo tie-breaking strategies search each plateau in decreasing and increasing order of the depth, respectively. Assume we are using $[f, h, *]$ sorting strategy. The lifo strategy always selects the most recently generated node within plateau $(f, h)$, and the behavior in the plateau is equivalent to depth-first search. Thus, lifo always selects a node in the largest depth, as depicted in Figure 5.1 (subfigure 2). Similarly, the behavior of fifo strategy in a plateau is equivalent to breadth-first search. Thus fifo always selects the nodes with the least depth (subfigure 3 ). Note that $[f, h$, lifo $]$ is equivalent to $[f, h,-d$, lifo $]$ and $[f, h, f i f o]$ is equivalent to $[f, h, d, f i f o]$.

The problem with these traditional strategies is that we have no knowledge regarding whether the goals are located close to or far from the entrance. Recall that since $f=f^{*}$, all goal nodes in the final plateau are optimal with respect to solution cost regardless of the depth. However, until we find a solution, we do not know how the goals are distributed among various depths. In some problem instances the goals can be concentrated around the entrance, while in other problem instances the goals can be concentrated at some large depth.

In the former case, fifo should perform well because its breadth-first behavior naturally focuses the search around the entrance, favoring the smaller depths. However, in the latter case, exhaustively searching the shallower depths can result in not finding any solutions within the time limit because fifo may never reach the depth where the goals exist. On the other hand, lifo behaves in a depth-fist manner, so it may reach solutions at deeper depths quickly, but risks missing solutions at shallower depths. Thus, both fifo and lifo tie-breaking are prone to failures due to pathological cases.

In order to avoid focusing the search at the wrong depths (too shallow/deep), the safest policy seems to be to simply diversify the depths which are being searched, in order to avoid any depth-based biases which could lead to pathological behavior. In our proposed depth diversification strategy, the nodes are inserted into buckets associated with depths, and upon expansion, search effort is distributed in a more balanced manner among various depths (Section 5.2 defines "more balanced" more precisely). Nodes are not "sorted" according to increasing or decreasing order of depth - instead, we try to "diversify" the node expansion within the plateau. We denote this depth diversification criterion as $\langle d\rangle$. For example, $[f, h,\langle d\rangle]$ first breaks ties according to $h$ values, then uses the $\langle d\rangle$ criterion to break ties in plateau $(f, h)$.

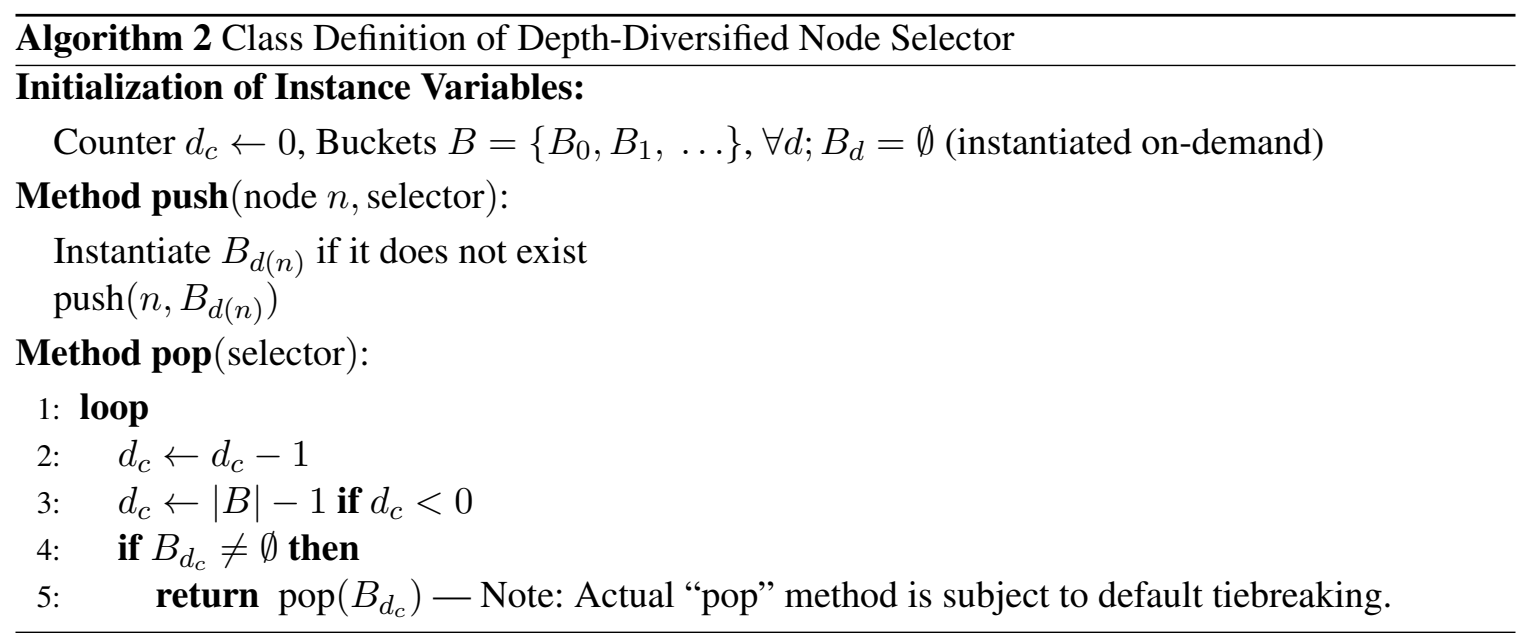


In order to diversify the expansion among depths, we simply iterate over the depth buckets (Algorithm 2). This iteration is managed by a Depth-Diversified Node Selector instance associated with each plateau (e.g. each of plateau $(1,0)$, plateau $(2,0)$, plateau $(2,1) \ldots)$. In order to select a single node from the OPEN list for expansion, we first select the plateau with the smallest key value, such as plateau $(f=5, h=1)$, as usual. This plateau is now represented by a selector instance, and we call pop(selector) method on this instance in order to obtain a node. Each instance holds an index $d_{c}$, the current depth (bucket index) selected in the last expansion, initialized to 0 . On each call to pop(selector), the counter is decremented $\left(d_{c} \leftarrow d_{c}-1\right)$ and a node is further popped from $d_{c}$-th bucket, which can be a lifo, fifo or ro queue. When $d_{c}$ reaches below 0 , then $d_{c}$ is reset to the current largest depth in the plateau.

In an earlier, conference paper, we used a non-deterministic, randomized implementation of this idea (Asai \& Fukunaga, 2016), which does not have this counter and pops a node from a randomly selected bucket $\left(B_{\text {random }()}\right)$, but we use a deterministic implementation here because it facilitates the theoretical analysis below in Section 5.2 .

Depth-based diversification is significantly different from the ro strategy which simply selects a random node from the OPEN list. The uniform sampling behavior of ro behaves very similar to fifo, and is insufficient to achieve the level of diversity provided by our depth diversification tie-breaking, which is also already evidenced by the performance similarity between fifo and ro-based tiebreaking strategies (Table 3.1). This is because at any given point in the search, more nodes will tend to have shallower depths than deeper depths, and a uniform, random selection will, therefore, be biased to select a node with shallow depths. For example, imagine we have 100 nodes at depth $d=1$ and a single node at depth $d=2$. Since $r o$ does not consider the depth, the chance of expanding $d=2$ is only $1 / 101$. This probability does not improve until a sufficient number of expansions decreases the number of nodes in $d=1$. In contrast, our depth diversification policy expands nodes at $d=1$ and $d=2$ with equal probability.

Depth-based tie-breaking does not affect the order of node expansion when there are no remaining ties after the higher priority tie-breaking criteria, in which case all nodes have depth 0 . More formally:

Lemma 1. If all edge costs are positive, then $d(n)=0$ for every node $n$ expanded by $A^{*}[f, h,\langle d\rangle, *]$.

Proof. Let $n$ be a child of a node $m$. Regardless whether the parent $m$ of the node $n$ is newly assigned, updated, or the old parent is kept in line 10 of Algorithm 1, the invariant $g(n)=g(m)+$ $\operatorname{cost}(m, n)>g(m)$ holds because $\operatorname{cost}(m, n)>0$, and therefore $f(n)-h(n)>f(m)-h(m)$. This means that either $f(n) \neq f(m)$ or $h(n) \neq h(m)$, so $d(n)=0$.

Theorem 1. If all edge costs are positive, then $A^{*}[f, h,\langle d\rangle, *]$ expands nodes in the same order as $A^{*}[f, h, *]$ (where “*” is any criterion).

Proof. By Lemma 1, all nodes expanded by $A^{*}[f, h,\langle d\rangle, *]$ have depth 0 , and all nodes are in the same depth bucket in Algorithm 2, so $A^{*}[f, h,\langle d\rangle, *]$ expands nodes in the same order as $A^{*}$ $[f, h, *]$ regardless of the criterion $*$.

\subsection{Tie-Breaking within Depth Buckets}

Depth diversification cannot be a default tie-breaking by itself. Consider a tie-breaking strategy such as $[f, h,\langle d\rangle]$ which applies a depth-diversification tie-breaking. After the $\langle d\rangle$ criterion is applied, 
there may be multiple nodes within the same depth bucket, so a default tie-breaking criterion is still necessary to break ties among them. Thus, we should, for example, apply one of lifo, fifo or ro (random order) criteria after the $\langle d\rangle$ criterion.

There are two concerns about this default tie-breaking criteria. First, the default tie-breaking behavior is still susceptible to accidental biases, e.g., names/orders of action schema in the PDDL domain definition (Vallati, Hutter, Chrpa, \& McCluskey, 2015). Second, in addition to accidental biases, there may be some nontrivial biases that require sophisticated algorithms to be removed.

Recent work showed that the performance of a satisficing planner can be significantly affected by the order in which actions appear in a PDDL file (Vallati et al., 2015). However, the conference version of this paper (Asai \& Fukunaga, 2016) showed that the effect of such an accidental bias is not statistically significant in cost-optimal search, by comparing the performance on several sets of randomly "mangled" domains whose action names are replaced with random strings. Moreover, the ro default tie-breaking should be unaffected by such an accidental bias. Thus, we believe it is safe to claim that the experimental results in this paper are not a product of such accidental biases.

In addition to accidental biases, there may be other nontrivial biases such as some form of symmetry among states which can be removed using some tie-breaking criterion $X$. Such a criterion can be applied after the depth criterion but before the default criterion, resulting in a sorting strategy $[f, h,\langle d\rangle, X, f i f o]$. Candidates for $X$ may be related to pruning techniques such as Symmetry Breaking (Fox \& Long, 1998; Pochter, Zohar, \& Rosenschein, 2011; Domshlak, Katz, \& Shleyfman, 2013) or Partial Order Reduction (Hall, Cohen, Burkett, \& Klein, 2013; Wehrle, Helmert, Alkhazraji, \& Mattmüller, 2013). While these are usually described as "pruning techniques", they can also be interpreted as strong bias removal mechanisms because they seek to prune redundant nodes, and redundancy causes a biased search effort. For example, imagine we have a set of nodes $S=\left\{a_{1}, a_{2}, a_{3}, a_{4}, b, c, d\right\}$ where $A=\left\{a_{1}, a_{2}, a_{3}, a_{4}\right\}$ are "redundant" according to some measure (e.g. by Symmetry, Partial-Order). If a search algorithm expands $S$ by random selection, it favors the group $A$ by giving 4 times larger chance of expansion than each of $b, c$ or $d$. Despite this similarity, search diversification is weaker than pruning methods because diversification can only delay the expansion of nodes sharing the similar attributes (such as depth), not prune the nodes.

\subsection{Theoretical Characteristics of the Depth Distribution}

We give further insight into the search behavior of our implementation of depth-based diversification. In depth-based diversification, although it is possible to select from a randomly selected depth bucket, as was done in an earlier conference paper (Asai \& Fukunaga, 2016), the implementation used in this paper performs a deterministic, round-robin sampling from the available depth buckets as described in Algorithm 2. We are particularly interested in how the nodes selected for expansion are distributed among the various depths in a plateau region. Assume that a search algorithm is searching a plateau region $P$. The precise definition of $P$ depends on the higher-level sorting strategy e.g. $[f, h,\langle d\rangle]$ or $[f,\langle d\rangle]$. Using a simplified model where this $P$ forms a forest (a set of disjoint trees), we can analyze the number of expansions in a particular depth can be represented by a simple formula.

In the discussion below, we first assume that $P$ forms a forest of a fixed branching factor $w \geq$ 2 (forest assumption), rather than a graph with an indefinite number of successor nodes. In the later experiments, we show this is a fairly accurate model. We also assume that no depth bucket is exhausted due to the expansion (no-exhaustion assumption). This implies that there are a sufficiently 
large number of nodes in depth $d=0$ so that depth 0 is not exhausted, which may cause fifo default tiebreaking to fail due to the heavy bias to the shallow depth. We provide a condition for this assumption to hold within this section. An example of running depth diversification with $w=3$ is depicted in Figure 5.2



Figure 5.2: Depth Diversification applied to a plateau with forest assumption and no-exhaustion assumption.

Let $D \geq 0$ be the current largest depth of the nodes found in $P$ so far. This is equal to $|B|-1$ in Algorithm 2, the size of the buckets in Depth-Diversified Node Selector instance. An expansion of a node at depth $D$ results in $w$ more nodes with depth $D+1$ on the same plateau $P$. These children are all newly generated because by the forest assumption, each child has a single incoming edge. Since the expansion is diversified by a sequence of iterations from the current largest depth to 0 , when the current largest depth of the plateau is $D$, the number of iteration executed so far is also $D$ because at the beginning of each iteration the largest depth is increased by 1 . Therefore, at the end of the $D$ 'th iteration, each depth $d$ has been expanded exactly $D-d$ times, with $D(D-1)$ expansions in total. In Figure 5.2, after iteration 2, depth $d=0$ is expanded twice and depth $d=1$ is expanded once.

It also means that a sufficient condition for no-exhaustion assumption to hold until the end of the $D$ 'th iteration is that the initial number of nodes in depth 0 is at least $D$. If there are at least $D$ nodes in depth 0 , depth 0 is trivially never exhausted until the $D$ 'th iteration. Also, no depth buckets in depth $d>0$ will be exhausted because each bucket has $w(D-d+1)$ generated nodes in total (i.e. OPEN+CLOSED) while the expansion has happened only $D-d$ times. The number of nodes in each bucket $(w(D-d+1))$ follows from the fact that depth $d-1$ is expanded $D-(d-1)$ times in the preceding $D$ iterations. Since $w \geq 2, w(D-d+1) \geq 2(D-d)+2>D-d$.

If there are no solutions, every depth-selection criterion, including least depth selection (fifo) or largest depth selection (lifo), expands the same set of nodes and results in the same distribution as depth diversification. For example, if the number of nodes in depth 0 is $D$, each $d$ is expanded $D w^{d}$ times. However, their online characteristics are different. Under our assumptions, the $D-d$ distribution of depth diversification is an invariant which holds at any point in the search until the 
solution is found. In contrast, in fifo, all nodes with $d<D-1$ are expanded, depth $d=D-1$ can take an arbitrary number of expansions $e \in\left[0, D w^{D-1}\right]$ and $d \geq D$ are not expanded at all. In lifo, for some $k \in\left[0, D_{\max }\right]$ (assuming the forest has a finite maximum depth $D_{\max }$ ), there can be a situation where all depths $d \in[0, k]$ get only 1 expansion each while all nodes in depths $d \in$ $\left[k+1, D_{\max }\right]$ are expanded. In this case, the number of expansions in $d \in\left[k, D_{\max }\right]$ is exponential to $D_{\max }-k\left(\sum_{i=k}^{i=D \max } w^{i-k}=\frac{1-w^{D \max -k+1}}{1-w}\right)$ while the number of expansions in $d \in[0, k-1]$ is linear to $k$ (i.e. $k-1$ ). Such an imbalance during the search causes the pathological behavior mentioned above.

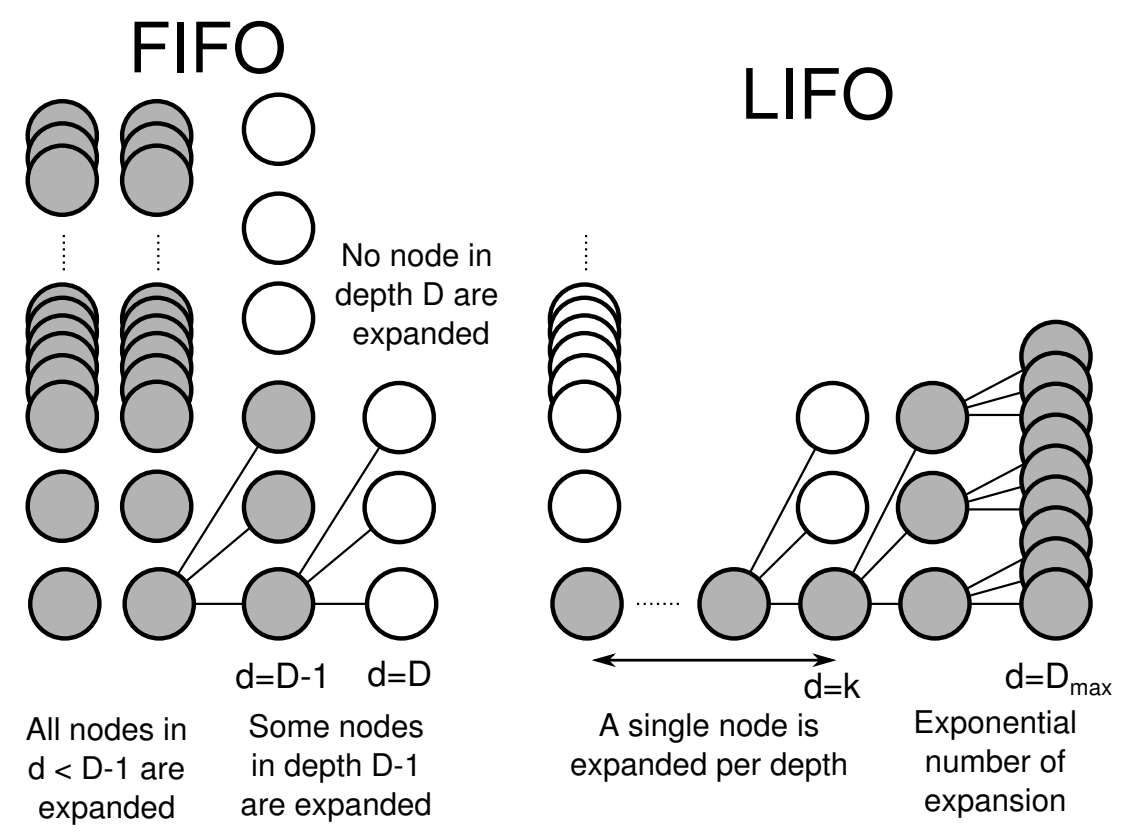

Figure 5.3: FIFO and LIFO applied to a plateau with forest assumption and no-exhaustion assumption.

\section{Evaluating Depth-Based Tie-Breaking}

We compared the performance of standard tie-breaking methods to depth-based tie-breaking methods. These all use $h$ as the second-level sorting criterion and either fifo, lifo or ro (random order) default tie-breaking criterion. The only difference is the presence of the third, depth-diversification criterion.

Experiments are conducted on 1104 standard IPC benchmark instances from 35 domains and 620 Zerocost instances from 28 domains (see Section 3 and Section 4 for full lists of these domains). The basic experimental settings are the same as the previous ones: Each experiment uses the Fast Downward planner using $A^{*}$ search and either the LMcut heuristic or M\&S heuristic. Each experiment is run for 5 minutes excluding SAS translation time, with 4GB memory constraints.

We first show the summary results of these experiments (Table 6.1). Overall, depth-based tiebreaking tends to show larger coverages than the standard tie-breaking strategies. Interestingly, 
when the depth diversity criterion $\langle d\rangle$ is used, the performance relationship between lifo and fifo seems to flip: fifo tends to perform better than lifo in Zerocost domains for both LMcut and M\&S heuristics (299 vs 279 for LMcut, 317 vs 303 for M\&S). Also, ro (random order) outperforms both fifo and lifo. In the following, we describe and discuss each experiment. Detailed data tables are in the Appendix (Section A).

\begin{tabular}{|l|cc|cc|} 
Sorting Criteria & $\begin{array}{c}\text { Zerocost(620) } \\
\text { LMcut }\end{array}$ & $\begin{array}{c}\text { Zerocost(620) } \\
\text { M\&S }\end{array}$ & $\begin{array}{c}\text { IPC(1104) } \\
\text { LMcut }\end{array}$ & $\begin{array}{c}\text { IPC(1104) } \\
\text { M\&S }\end{array}$ \\
Standard & & & & \\
{$[f, h$, fifo $]$} & 256 & 280 & 558 & 491 \\
{$[f, h$, lifo $]$} & 279 & 301 & 565 & $\mathbf{4 9 6}$ \\
{$[f, h$, ro $]$} & $261.9 \pm 1.4$ & $287.7 \pm 3.2$ & $558.9 \pm 2.1$ & $489.4 \pm 1.0$ \\
Depth-based & & & & \\
{$[f, h,\langle d\rangle$, fifo $]$} & 284 & 302 & 571 & 487 \\
{$[f, h,\langle d\rangle$, lifo $]$} & 264 & 288 & $\mathbf{5 7 5}$ & 487 \\
{$[f, h,\langle d\rangle$, ro $]$} & $\mathbf{2 8 8 . 1} \pm \mathbf{1 . 6}$ & $\mathbf{3 0 8 . 1} \pm \mathbf{2 . 1}$ & $571.4 \pm 1.7$ & $485.6 \pm 1.5$
\end{tabular} \mid

Table 6.1: Main summary results: Coverage comparison (number of instances solved in 5min, 4GB, LMcut/M\&S heuristics) between standard tie-breaking and depth-based tie-breaking $(\langle d\rangle)$. When LMcut is used, $\langle d\rangle$ outperforms standard strategies both in IPC instances (1104 problems total) and Zerocost instances (620 problems total). When M\&S is used, $\langle d\rangle$ outperforms standard strategies in Zerocost instances. Bold shows the best configuration.

Table A.3 and Table A.4 show the number of Zerocost instances (out of 620) solved by LMcut and $\mathrm{M} \& \mathrm{~S}$ heuristics. In these Zerocost domains, our proposed method outperforms the traditional tie-breaking methods in both heuristics. Significant improvements were observed in 10 domains when using LMcut, and 7 domains when using M\&S.

Table A.5 shows the number standard IPC benchmark instances (out of 1104) solved by the configuration using LMcut heuristics. Depth-based tie-breaking $(\langle d\rangle)$ achieves impressive results on Openstacks (fifo: $2 \rightarrow 8$, lifo: $3 \rightarrow 12$, ro : $3.9 \rightarrow 10$ ) and Cybersec (fifo: $11 \rightarrow 18$, ro : $11.7 \rightarrow 18$ ) because these domains contain many instances of 0 -cost edges (See Figure 3.2). Most other instances are unaffected by depth-based tie-breaking. Thus, depth-based tie-breaking yields better performance in the domains with 0 -cost actions, without sacrificing performance in other domains.

In contrast, Table A.6 shows that depth-based tie-breaking degrades the performance of the configuration using M\&S when applied to 1104 standard IPC benchmark instances. This result can be explained as follows. First, similar to the case of LMcut, Openstacks coverage improved for fifo $(15 \rightarrow 19)$ and ro $(15.4 \rightarrow 19)$, which is expected according to our analysis of Zerocost domains. Although there was no improvement on Cybersec, this is because the coverage of Cybersec is 0 in all M\&S configurations, regardless of tie-breaking. Thus, the positive contribution of depth diversification to the overall score was limited for M\&S compared to LMcut.

Second, with M\&S, performance degraded across a wide range of domains due to the low-level overhead of depth-based tie-breaking (i.e., updates to the depth-based bucket data structures). As 
shown in Figure 6.1, when depth-based tie-breaking was used, the node evaluations rate significantly decreased with the M\&S heuristic, while node evaluation rate decreased much less for LMcut. This is because the $M \& S$ heuristic is implemented as an efficient table lookup, and $M \& S$ is able to evaluate an order of magnitude larger number of nodes compared to LMcut. Thus, even the relatively small overhead incurred by depth bucket updates decreases the node evaluation rate enough to noticeably degrade $M \& S$ performance. Figure 6.2 shows a cumulative coverage plot which shows the number of node evaluations required to solve IPC instances. According to Figure 6.2, the number of evaluations required to solve IPC instances for $[f, h, *]$ and $[f, h,\langle d\rangle, *]$ were almost identical, which is expected because IPC instances mostly consist of instances with only positive-cost actions which are unaffected by depth-based tie-breaking (as predicted by our analysis in Section 5). This shows that the coverage degradation on IPC instances when using depth diversification is caused by the low-level overhead.
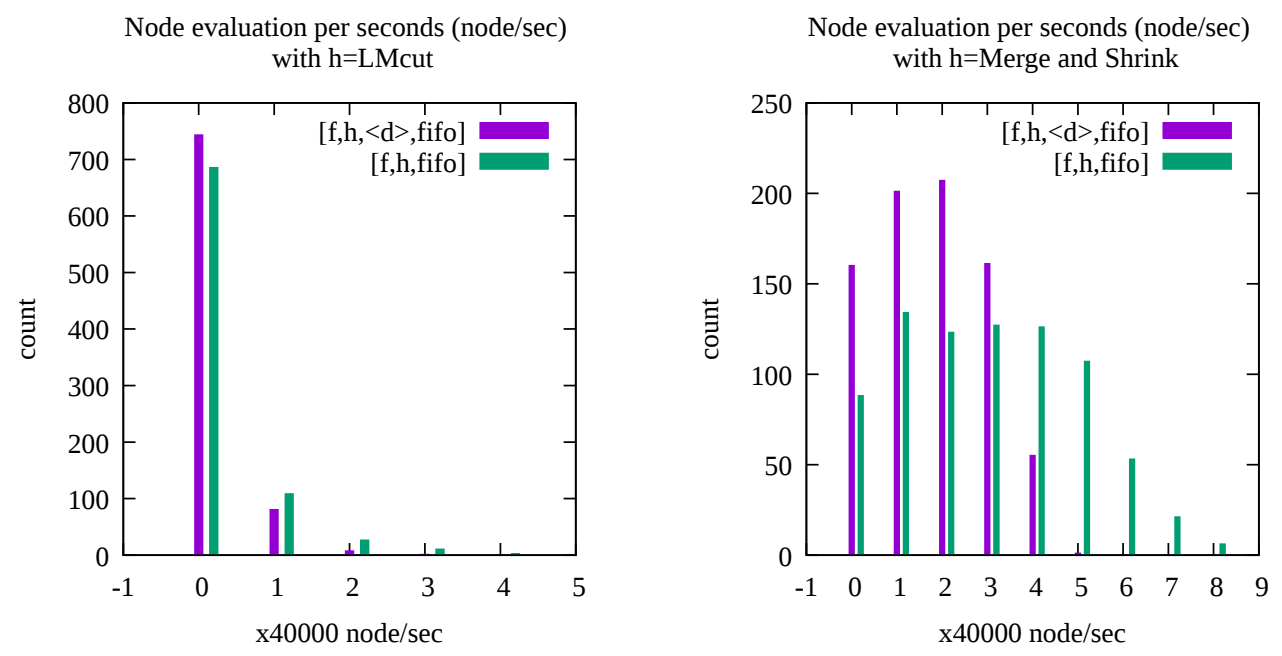

Figure 6.1: Histogram comparing the node evaluation ratio (node/sec) between standard tiebreaking $([f, h, f i f o])$ and depth-based tie-breaking $([f, h,\langle d\rangle, f i f o])$ on LMcut and M\&S heuristics. This plot includes both IPC and Zerocost instances. (See Appendix Figure A.1 for the data on $[f, h$, lifo $]$ vs. $[f, h,\langle d\rangle$, lifo $]$.) On M\&S, compared to LMcut, node evaluation rate more often becomes slower when depth is enabled. This is because the node evaluation of $M \& S$ is an order of magnitude faster than LMcut, and the overhead of managing depth-based tie-breaking queue becomes significant.

Finally, the per-domain results for Zerocost domains (Tables A.3-A.4 show that $\langle d\rangle$ can cause both improvement and degradation (despite the total coverage improvement). This is natural considering that depth-diversification is designed to be a conservative, domain-independent strategy which is designed to avoid worst-case pathological behaviors. Overall, $\langle d\rangle$ tends to perform well, but the best-performing strategy on particular domain varies - for example, fifo is the best in airport-fuel with LMcut, while lifo is the best in freecell-move with LMcut. An adaptive tie-breaking which selects the tie-breaking strategy for a given domain is discussed in Section 8.3 . 



Figure 6.2: Cumulative coverage ( $y$-axis) vs the number of evaluated nodes ( $x$-axis), on IPC instances solved by both $[f, h, *]$ and $[f, h,\langle d\rangle, *]$ where $h=$ M\&S. Left: fifo, Right: lifo.

\subsection{Search Behavior Within a Plateau}

To understand the behavior of depth-based policies, we plotted histograms of the depths of search nodes evaluated by several tie-breaking strategies in the final plateau plateau $\left(f^{*}, 0\right)$ until the solution is found. We plotted a depth-based strategy $[f, h,\langle d\rangle, f i f o]$, as well as the standard strategies $[f, h, f i f o],[f, h$, lifo $]$ and a single run of randomized strategy $[f, h, r o]$.

In order to obtain the data for the strategies which do not use depth-based tie-breaking ( $[f, h, f i f o]$, $[f, h, l i f o],[f, h, r o])$, we added some instrumentation to these strategies so that, the depth of each of the expanded nodes is computed, although they do not affect the search behavior. Note that this instrumentation, which adds some runtime overhead, was not used in the performance comparison experiments above, and were only used for this experiment, which analyzes search behavior.

Figure 6.3 (as well as Figures A.2 - A.3 in the Appendix) show the results on exemplary instances from various Zerocost domains. We do not show some domains where we did not observe any depths greater than 3 , in which case both the depth metric and lifo/fifo/ro have a negligible impact on search performance. We observed very similar results across a wide range of domains as shown in the figures. This indicates that the depth metric accurately describes the behavior of each tie-breaking criterion.

For example, consider the first figure, which plots depths searched on depot-fuel, p07. The $[f, h$, lifo $]$ plot shows that the depth-first behavior results in deeper search $\left(\approx 10^{3}\right)$, while only a handful of nodes are expanded at intermediate depths (usually once). Thus, lifo's depth-first behavior is prone to missing the key branch at intermediate depths that may lead to solutions earlier. On the other hand, the breadth-first behavior of $[f, h, f i f o]$ often gets stuck spending an excessive amount of time searching around the plateau entrance (expanding $\approx 10^{3}$ nodes at depth 10 ).

Also, we noticed that the node distribution of the global randomization $[f, h, r o]$ is very similar to $[f, h, f i f o]$. This shows that ro actually behaves very similar to fifo, which is consistent with the 
previous performance comparisons in Section 3 and our observation regarding ro in Section 5 . Thus, the overall behavior of ro tends to be similar to fifo, and naive randomization does not solve the problem of heavy bias for shallower depth nodes.

In contrast, $[f, h,\langle d\rangle, f i f o]$ is balancing the search at various depths. The yellow curve representing $[f, h,\langle d\rangle, f i f o]$ tends to be almost flat at shallow depths while gradually decreasing the number of nodes at larger depths. Moreover, its node distribution almost accurately follows $D-d$, a theoretical model from Section 5.2 which applies the simplified assumption that the plateau is a forest with a fixed branching factor. $D$ denotes the largest depth of the unexpanded nodes in the final plateau, which is 1 larger than the largest depth of the expanded nodes.

The discrepancy of the $[f, h,\langle d\rangle$, fifo $]$ curve from the theoretical prediction $D-d$ can be caused by the following factors: First, the outdegree of each node in the graph may not be uniform across the search space. Second, some depth buckets could be exhausted, as depicted in the $[f, h, f i f o]$ line which shows that all nodes in the shallower depths are expanded while the line is still below $D-d$. Since $[f, h, f i f o]$ exhaustively expands the nodes in shallower depth, the number of expansion by $[f, h, f i f o]$ in the shallower depths constitutes an upper bound, which may be below $D-d$.

Next, Figure 6.4 shows the same results on the standard IPC Openstacks and Cybersec domains. The Openstacks results were similar to those of the Zerocost domains. In Cybersec, we found that the performance improvement was not due to the number of nodes in plateau $\left(f^{*}, 0\right)$, because all tie-breaking strategies have generated only a small number of such nodes before the solution was found. Instead, we observed a large difference in the depth distributions in non-final plateaus plateau $\left(f^{*}, h\right), h \neq 0$ caused by the difference of tie-breaking. Note that depth diversification is always applied regardless of $f$ or $h$ values. This suggests that most children of the nodes in plateau $\left(f^{*}, h\right)$ have $f$ value larger than $f^{*}$ or stays in plateau $\left(f^{*}, h\right)$, and the planner is struggling to find nodes with better $h$. Due to the unbiased search, the depth-based strategy has a better chance of improving $h$ values, finding a node in plateau $\left(f^{*}, 0\right)$ more quickly. This shows that considering depth can also help the search in non-final plateaus to find the nodes in the next plateau. Similar phenomena were observed in several other instances and domains, e.g., depot-fuel, driverlog-fuel, zenotravel-fuel, floortile-ink, mprime-succumb, storage-lift (Figure A.4 in Appendix).

Note that the small number of nodes in plateau $\left(f^{*}, 0\right)$ in this experiment does not contradict the results in Figure 3.2, which shows that the number of such nodes is quite large. This is because, while in Figure 3.2 the search continues until expanding all nodes in the final plateau, in this experiment the search stops when the first solution is found - Figure 3.2 was intended to show the size of the entire final plateau, while Figures 6.3-6.4 were meant to show the actual search behavior. If we continue the search until exhausting the final plateau, all tie-breaking strategies will expand the same set of nodes (in different orders), so we would obtain plots similar to Figure 3.2 regardless of the tie-breaking strategy. 

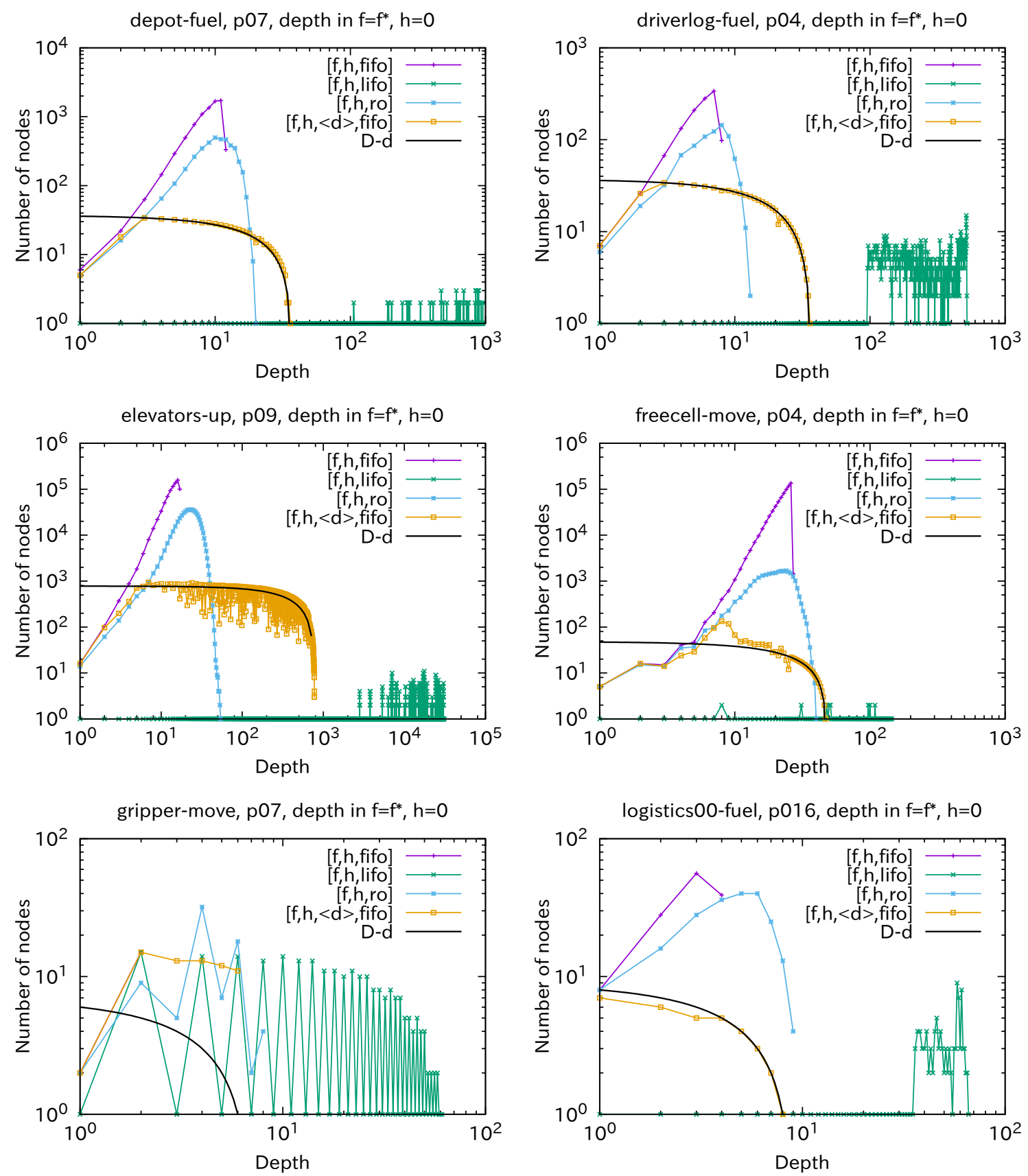

Figure 6.3: Number of nodes ( $y$-axis) expanded per depth ( $x$-axis) in the final plateau with different tie-breaking strategies. Both axes are in logarithmic scale. 

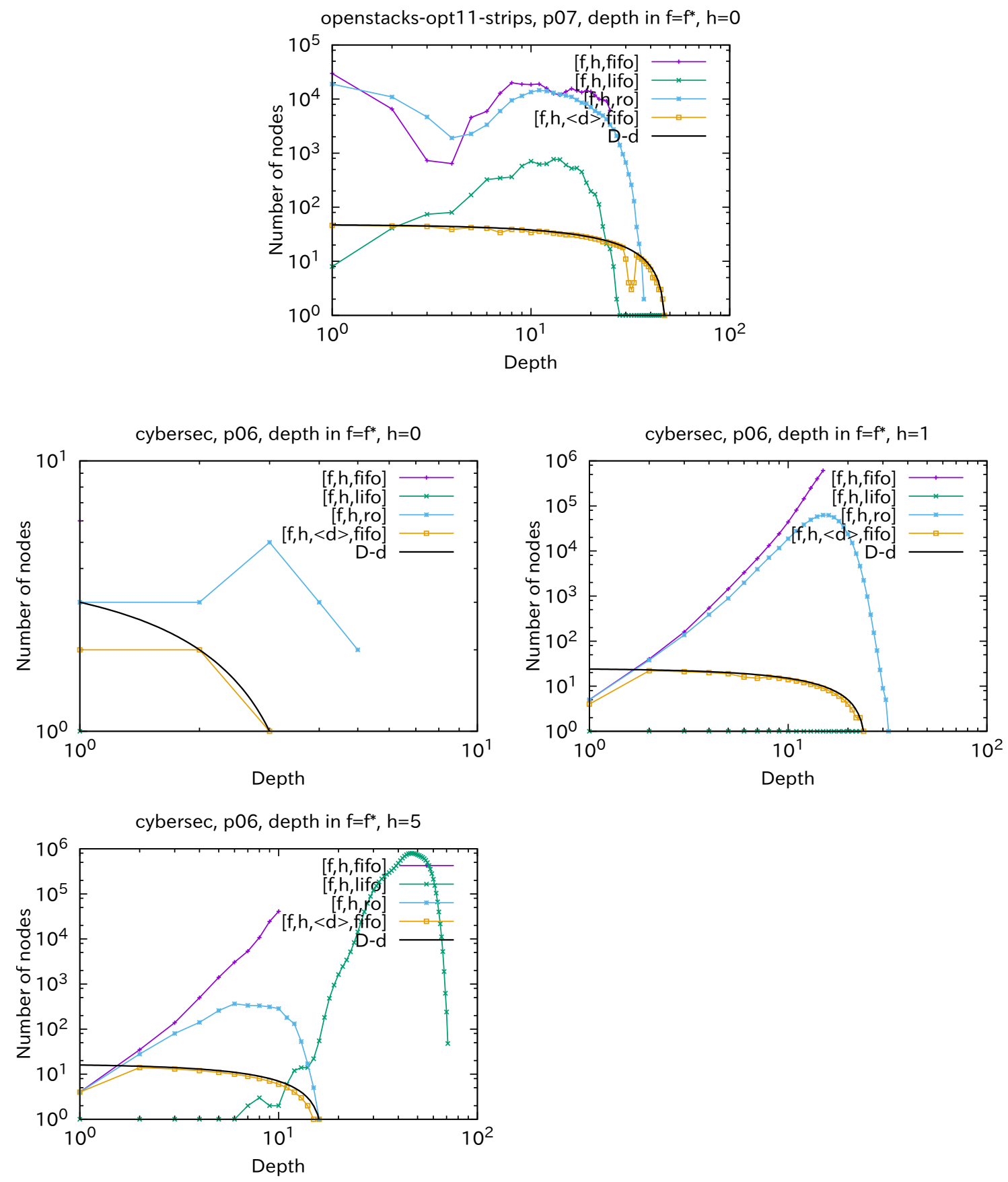

Figure 6.4: Depth distribution of Openstacks and Cybersec instances in the final (plateau $\left(f^{*}, 0\right)$ ) and non-final plateaus (plateau $\left(f^{*}, h\right), h \neq 0$ ). In Cybersec p06, although the number of nodes generated in plateau $\left(f^{*}, 0\right)$ is small, fifo and ro behaved poorly on plateau $\left(f^{*}, 1\right)$, and also lifo behaved poorly on plateau $\left(f^{*}, 5\right)$. 


\section{A New Framework: $A^{*}$ as a Series of Satisficing Search Episodes}

So far, we have shown that by carefully analyzing search within an $f$-cost plateau, we were able to develop an effective knowledge-free, depth-based tie-breaking method which can significantly improve search performance on domains with 0 -cost actions. We now propose a more general framework which underscores the importance of tie-breaking in $A^{*}$. Cost-optimal search can be seen as a series of satisficing searches on each plateau. In this framework, the problem of tie-breaking can be reduced to a satisficing search.

While $A^{*}$ requires the first sorting criterion $f$ to use an admissible heuristic in order to find an optimal solution, there are no requirements on the second or later sorting criterion. This means that the search within the same $f$ plateau can be an arbitrary satisficing search ${ }^{2}$ without any cost minimization requirement. For example, if we ignore the first sorting criterion in the standard admissible strategy $[f, h, f i f o]$, we have $[h$, fifo], which is exactly the same configuration as a Greedy Best First Search (GBFS) using fifo default tie-breaking. This means that within a particular $f$-cost plateau, $[f, h, f i f o]$ is performing a satisficing GBFS. As another example, the reason for the poor performance of $[f, f i f o]$ is clearly that it is running $[f i f o]$, an uninformed satisficing breadth-first search in the plateau.

From this perspective, we can reinterpret $A^{*}$ as in Algorithm 3: $A^{*}$ expands the nodes in bestfirst order of $f$ value. When the heuristic function is admissible, the $f$ values of the nodes expanded by $A^{*}$ never decreases during the search process. Thus, the entire process of $A^{*}$ can be considered as a series of search episodes on each plateau $(f)$. The search on each plateau terminates when the plateau is proven to contain no goal nodes (UNSAT), or when a goal is found (SAT). When the plateau is UNSAT, then the search continues to the plateau with the next smallest $f$ value. Figure 7.1 also illustrates this framework.

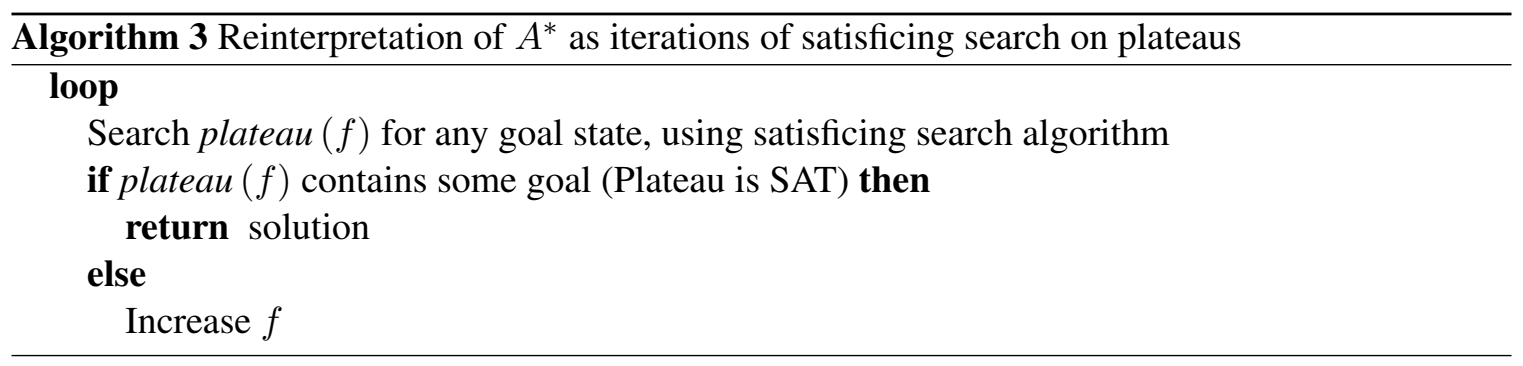

This is somewhat similar to the standard approach to model-based planning using SAT/IP/CP solvers (Kautz \& Selman, 1992; van den Briel \& Kambhampati, 2005), based on an iterative strategy where a planning problem is converted to a corresponding constraint satisfaction problem with a finite horizon $t$ (plan length / makespan). The search starts from the horizon 0 and tests if the problem is satisfiable. If not, then it increases the horizon, add constraints excluding solutions below $t$, and retests the same problem with additional constraints for a new horizon $t+1$.

It is also reminiscent of the behavior of iterative deepening $A^{*}$ (Korf, 1985), which executes a series of satisficing searches with an $f$-cost limit which increases on each iteration. However, " $A^{*}$-as a sequence of satisficing search" differs from IDA* in that IDA*, in order to achieve linear

2. This refers to any algorithm which seeks a satisficing solution, as opposed to the "satisficing" track setting in IPC which also seeks to minimize the plan cost with anytime algorithms 


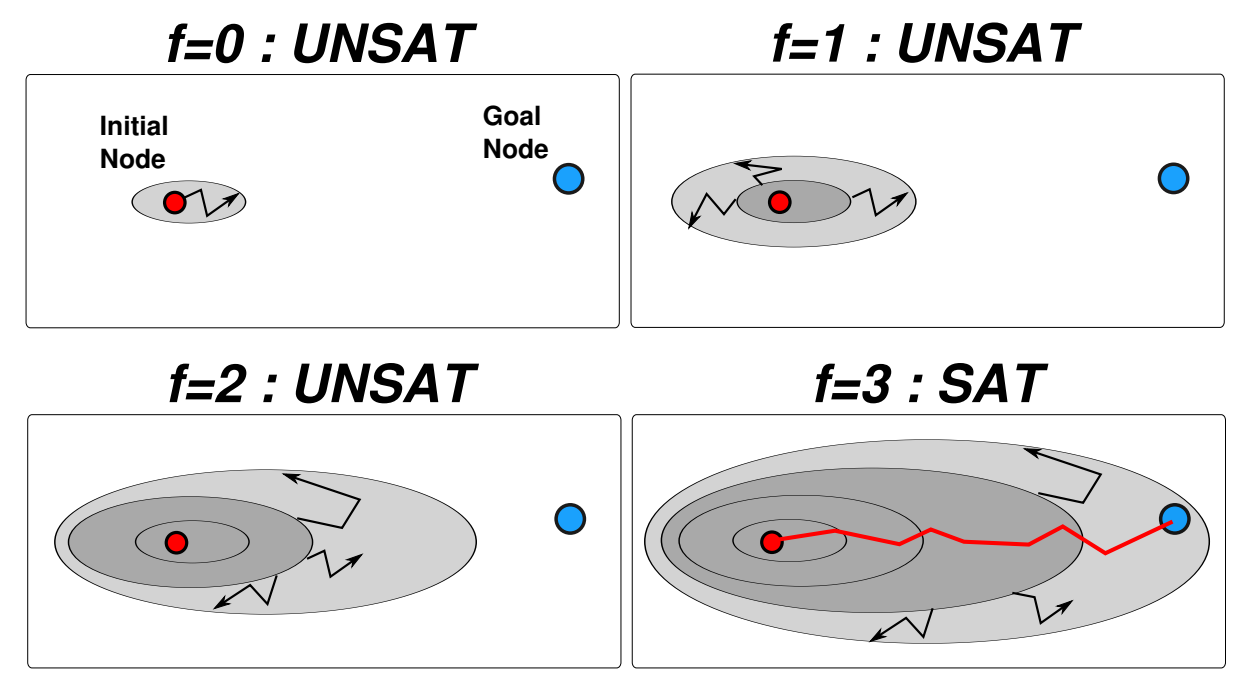

Figure 7.1: The concept of $A^{*}$ as a sequence of satisficing searches.

memory usage, repeats previous work on each iteration. Instead of searching a particular plateau in each iteration, IDA* searches through the union of several plateaus.

The framework of " $A^{*}$ as a series of satisficing searches" suggests that we can directly apply satisficing search techniques to optimal search using $A^{*}$, especially for each $f$-cost plateau search. In the following two subsections, as well as in the next section, we show that this framework (1) provides a better understanding of depth-diversification (Section 7.1), (2) allows us to prove the completeness of $A^{*}$ on infinite graph depending on the tie-breaking methods (Section 7.2), and (3) allows us to improve the performance of $A^{*}$ on Zerocost domains (Section 8 ).

\subsection{Depth Diversification as Satisficing Search}

Within this framework, the implementation of depth diversification can be viewed as a variant of the Type-based diversification approach (Xie, Müller, Holte, \& Imai, 2014), specifically tailored for Zerocost domains.

Xie et al. proposed type based buckets, an implementation of the OPEN list which partitions the nodes into buckets according to some set of key values (types). They proposed several types such as $\langle 1\rangle,\langle g\rangle,\langle h\rangle$ or $\langle g, h\rangle$. At each type-based expansion, a randomly selected node from a randomly selected single bucket is selected. For example, with type $\langle g, h\rangle$, a node with $g=5$ and $h=3$ is put into a bucket $\langle 5,3\rangle$. This mechanism diversifies the search so that it tries to expand the nodes with various distances from the initial state and various distances from a goal state.

They then proposed Type-GBFS, which alternates the expansion between normal GBFS with a $[h, f i f o]$ sorting criteria and type-based expansion. This alternating framework addresses a weakness of GBFS: GBFS is solely guided by the heuristic function $h$, and heuristic errors in $h$ can easily misguide GBFS to spend all of its time in the wrong part of the search space - GBFS can become trapped due to heuristic error and cannot recover from the wrong decision until expanding all nodes in that branch. In the worst case, on infinite graphs, GBFS is not complete because it can be misdirected by the heuristic guidance forever (Valenzano \& Xie, 2016). In contrast, in Type-GBFS, the alternation with type-based expansion introduces exploratory behavior of nodes with low $g$ and 
high $h$, offering the possibility of escaping from heuristic error traps. As a result, Type-GBFS is probabilistically complete on infinite graphs (Valenzano \& Xie, 2016).

Type-GBFS was primarily evaluated in the context of satisficing search with no consideration of plan quality, and performance is solely evaluated according to coverage. Thus, Xie et al adopted a unit-cost domain model: All action costs are ignored and replaced with unit costs in their experiments in order to boost coverage (Xie et al., 2014). This is a commonly used technique for satisficing search which is also used in the first iteration of LAMA2011 (Richter et al., 2011).

In our framework of $A^{*}$ as a sequence of satisficing searches, depth diversification after $h$ tiebreaking $([f, h,\langle d\rangle])$ can be viewed as the combination of (1) an implicit transformation of all 0-cost edges within a single plateau $(f, h)$ to unit-cost edges, and (2) a pure type-based exploration within that plateau (unlike Type-GBFS, which alternates GBFS and type-based buckets).

The notion of depth counts the number of 0-cost actions, which does not change the $f$ value and $h$ value, on the path from the entrance to the current plateau, to the current node. Thus, depthdiversification treats the problem of finding an exit from a particular plateau as a unit-cost satisficing search problem - the depth is analogous to a $g$-value which is calculated with unit costs and is restricted to a particular plateau.

Depth diversification for tie-breaking in admissible $A^{*}$ has a different purpose and context from Type-GBFS for satisficing search, and differs as follows. First, depth diversification is focused on finding a satisficing plan within a single plateau and on solving domains with 0-cost actions. Therefore, depth diversification is applied after the sorting by $h$. In contrast, type buckets are global type buckets have no preceding sorting criteria, and all open nodes are stored in these buckets. Type-GBFS then alternates type buckets and sorting by $h$, not applying them in a cascade manner.

Nevertheless, the close relationship between depth diversification for admissible $A^{*}$ and TypeGBFS for satisficing search is important. It shows that if we apply our framework of " $A^{*}$ as a series of satisficing searches", we can directly use ideas which have been previously proposed for satisficing search within each $f$-cost plateau search.

\subsection{Completeness of $A^{*}$ on an Infinite Graph}

Similarly, we can use this framework for analyzing the completeness of $A^{*}$ on infinite graphs with respect to various tie-breaking criteria. First, $A^{*}$ is complete on finite graphs regardless of the tiebreaking strategy (Hart et al., 1968). However, if the graph is infinite, the completeness of the algorithm depends on tie-breaking. We consider several cases depending on which plateau is infinite. We only need to consider plateaus for $f \leq f^{*}$ because $A^{*}$ does not expand the nodes in plateau $(f)$ for $f>f^{*}$.

Definition 1. A graph is infinite when the number of nodes in the graph has no upper bound.

Proposition 1. If any plateau $(f)$ for $f<f^{*}$ is infinite, then $A^{*}$ does not terminate.

Proof. Algorithm 3 requires proving the UNSAT-isfiability (that there is no solution) of all non-final plateaus, plateau $(f)\left(\forall f<f^{*}\right)$, so if any of them are infinite, $A^{*}$ does not terminate.

The remaining cases assume that the following two conditions hold: plateau $(f)$ is finite $\forall f<$ $f^{*}$, and plateau $\left(f^{*}\right)$ is infinite. Under this assumption, the completeness of $A^{*}$ using tie-breaking $\left[f\right.$, criterion ${ }_{2}, \ldots$ criterion $\left._{k}\right]$ depends on the completeness of the satisficing search algorithm corresponding to $\left[\right.$ criterion $_{2}, \ldots$ criterion $\left._{k}\right]$ on plateau $\left(f^{*}\right)$. For the standard tie-breaking criteria, we can apply previously known results. 
Theorem 2 (Valenzano and Xie (2016)). $\varepsilon$-greedy node selection (Valenzano, Schaeffer, Sturtevant, $\&$ Xie, 2014) is probabilistically complete on an infinite graph, i.e., the probability of finding a solution approaches to 1 when the number of expansion $t$ approaches $\infty$.

Corollary 1. $A^{*}$ with Random Order tiebreaking $[f$, ro $]$ is probabilistically complete on an infinite graph when plateau $(f)$ is finite for all $f<f^{*}$.

Proof. $[f, r o]$ is an instance of $A^{*}$ using $[r o]$ as a satisficing algorithm for plateau-search. Since $[r o]$ is a special case of $\varepsilon$-greedy node selection with $\varepsilon=1,[r o]$ is also probabilistically complete on an infinite plateau $\left(f^{*}\right)$.

Breadth-first search is complete when the graph has a finite branch factor below the solution depth. Since FIFO tiebreaking $[f$, fifo $]$ applies breadth-first search to plateau $\left(f^{*}\right)$, it follows that

Proposition 2. $A^{*}$ with FIFO tiebreaking $[f, f i f o]$ is complete on an infinite graph when plateau $(f)$ is finite $\forall f<f^{*}$ and the maximum outdegree of the nodes is finite in plateau $\left(f^{*}\right)$ below the solution depth.

LIFO tie-breaking behaves equivalently to a depth-first search with duplicate detection (DFSdup) on plateau $\left(f^{*}\right)$. Assuming a fixed successor ordering, we get the following:

Proposition 3. $A^{*}$ with LIFO tiebreaking $[f$, lifo $]$ is complete on an infinite graph iff plateau $(f)$ is finite for all $f \leq f^{*}$.

Proof. If plateau $\left(f^{*}\right)$ is infinite, then either the maximum depth or the maximum outdegree of the nodes is infinite (has no upper bound). If the maximum depth has no upper bound, DFS-dup requires an arbitrary longer runtime before the first backtracking unless the solution is found before it. If the maximum outdegree has no upper bound, there is a successor ordering which forces DFS-dup to search all subtrees that do not contain solutions, and the size of the subtrees has no upper bound. If both the maximum depth and the maximum outdegree are finite, then plateau $\left(f^{*}\right)$ is finite and DFS-dup is complete. Combined with Proposition 1. LIFO tie-breaking requires a finite plateau $(f)$ for all $f \leq f^{*}$.

Finally, we show the completeness of our iteration-based depth diversification in Algorithm 2 .

Theorem 3. $A^{*}$ with Depth Diversification $[f,\langle d\rangle, *]$ is complete when plateau $(f)$ is finite $\forall f<f^{*}$ and the maximum outdegree of the nodes is finite in plateau $\left(f^{*}\right)$ for depths below the solution depth.

Proof. Any solution must have a finite depth $d^{*}$ on plateau $\left(f^{*}\right)$. On every iteration of the pop method of Algorithm 2 from the largest depth $D$ to the depth 0, each depth is expanded once. Since the maximum outdegree is finite, every node with depth $d \leq d^{*}$ will be expanded in a finite number of iterations.

\section{Tie-Breaking Using Distance-to-Go Estimates}

In the previous section, we proposed a framework which views cost-optimal $A^{*}$ search as a series of satisficing searches on each $f$-cost plateau, and argued that the problem of tie-breaking can be reduced to a satisficing search. We showed that the depth diversification tie-breaking criterion, which is highly effective on Zerocost domains, is in fact a case where a previously studied technique 
for satisficing search (type-based exploration) turns out to be highly effective when applied to tiebreaking. In this section, we push this insight further and propose another approach to improving the search performance in plateaus produced by Zerocost domains - using inadmissible distance-to-go estimates (heuristics) as a tie-breaking criterion within an admissible $A^{*}$ search.

Distance-to-go estimates are a class of heuristics which treat all actions as if they have unit cost. Even when 0-cost actions are present, these estimates can predict the number of operations required to reach a goal. In general, the estimates are inadmissible (unless the estimates are guaranteed to underestimate the number of required actions and all actions in the original domain have unit cost). Previous work on distance-to-go-heuristics has focused on their use for satisficing planning.

$A_{\epsilon}^{*}$ (Pearl \& Kim, 1982) is one of the earliest algorithms that combines distance-to-go estimates with the cost estimates. It is a bounded-suboptimal search which expands nodes from the focal list, the set of nodes with $f(n) \leq w \cdot f_{\min }$ where weight $w$ serves as a suboptimality bound, similar to weighted $A^{*}$, and $f_{\min }$ is the minimum $f$ value in the OPEN list. While $f$ is based on an admissible heuristic function, the nodes in the focal list are expanded in increasing order of an inadmissible distance-to-go estimate $\hat{h}$. Since the search does not follow the best-first order according to $f$, it is not admissible, and is instead $w$-admissible. One exception is the case of $w=1$ where the focal list is equivalent to the $f$ plateau and the expansion order in the focal list corresponds to the tiebreaking on plateaus. In our notation, this algorithm can be written ${ }^{3}$ as a BFS with the following sorting criteria:

$$
\left[\left\lceil\frac{f}{w \cdot f_{\min }}\right\rceil, \hat{h}, *\right]
$$

This notation is derived from the fact that the focal list "blur"s $f$ up to $w \cdot f_{\min }$. For example, when $w=2, f_{\min }=5$ and $f(n)=5,9,11$, then $\left\lceil\frac{f}{w \cdot f_{\min }}\right\rceil=1,1,2$ respectively.

Continuing this line of work, Thayer and Ruml $(2009,2011)$ evaluated various distance-togo configurations of Weighted $A^{*}$, Dynamically Weighted $A^{*}$ (Pohl, 1973) and $A_{\epsilon}^{*}$, where some configurations use distance-to-go as part of tie-breaking. This work focused on bounded-suboptimal search rather than cost-optimal search. Cushing et al. (2010) pointed out the danger of relying on cost estimates in a satisficing search by investigating " $\varepsilon$-cost traps" and other pitfalls caused by cost estimators for search guidance. Finally, the FD/LAMA2011 satisficing planner incorporates distance-to-go estimates in its iterated search framework (Richter et al., 2011). The first iteration of LAMA uses distance-to-go estimates combined with various satisficing search enhancements.

Benton et al. (2010) proposed an inadmissible technique for temporal planning where short actions are hidden behind long actions and do not increase makespan. Such actions cause "g-value plateaus", which are similar to the large plateaus caused by 0 -cost actions in sequential planning. They implemented an inadmissible heuristic function combined with distance-to-go estimates as an extension of Temporal Fast Downward (Eyerich, Mattmüller, \& Röger, 2009).

\subsection{Embedding Distance-to-Go Estimates in Admissible Search}

Although previous work on distance-to-go estimates assume a satisficing context, we show that distance-to-go estimates can be useful for cost-optimal search. Since the admissibility of the sorting strategy and the optimality of the solution are not affected by the second or later levels of sorting criteria, it is possible to use an inadmissible distance-to-go estimate in these subsequent sorting

3. However, an actual implementation may differ due to dynamic updates to $f_{\min }$. 
criteria without sacrificing the optimality of the solution found. This means inadmissible heuristics can be used for tie-breaking.

Let $h$ be an admissible heuristic function, and $\hat{h}$ be a distance-to-go variation of $h$, i.e., $\hat{h}$ uses essentially the same algorithm as $h$, except that while $h$ uses the actual action costs for the problem domain, $\hat{h}$ replaces all action costs with 1 . Since $h$ is admissible, multi-heuristic sorting strategies such as $[g+h, h, \hat{h}]$ or $[g+h, \hat{h}]$ are admissible.

Moreover, we can even use a multi-heuristic strategy which uses an inadmissible heuristic for tie-breaking which is unrelated to the primary, admissible heuristic $h$. For example, $\left[g+h^{\mathrm{LMcut}}, \hat{h}^{\mathrm{FF}}\right]$ is an admissible sorting strategy because the first sorting criterion $f=g+h^{\text {LMcut }}$ uses an admissible LMcut heuristic. Its second sorting criterion, the distance-to-go FF heuristic (Hoffmann \& Nebel, 2001), does not affect the admissibility of this entire sorting strategy.

A potential problem with sorting strategies which use multiple heuristics is the cost of computing additional heuristic estimates. For example, $\left[g+h^{\mathrm{LMcut}}, \hat{h}^{\mathrm{FF}}\right]$ requires more time to evaluate each node compared to a standard tie-breaking strategy such as $\left[g+h^{\text {LMcut }}, h^{\text {LMcut }}\right]$ because computing the $\hat{h}^{\mathrm{FF}}$ heuristic incurs significant overhead per node while the results of $h^{\mathrm{LMcut}}$ can be reused by a caching mechanism. When the inadmissible heuristic for tie-breaking is $\hat{h}$, i.e. a distance-to-go (unit cost) variant of the primary, admissible heuristic $h$, it may be possible to reduce this overhead to some extent by implementing $h$ and $\hat{h}$ so that they share some of the computation - this is a direction for future work.

\subsubsection{Combining Distance-To-Go Estimates With Default Tie-Breaking And DEPTH DIVERSIFICATION}

Tie-breaking using distance-to-go estimates can still leave a set of nodes which are equivalent up to the distance-to-go criterion (multiple nodes can have the same $f, h$, and $\hat{h}$ values), so additional level(s) of tie-breaking are necessary in order to select a single node. By adding a standard default criterion such as fifo, lifo, ro, we obtain a sorting strategy that imposes a total order. For example, $\left[f^{\mathrm{LMcut}}, \hat{h}^{\mathrm{FF}}\right.$, fifo $]$ applies fifo after the distance-to-go estimate $\hat{h}^{\mathrm{FF}}$.

Furthermore, it is possible to combine depth diversity based tie-breaking with distance-to-go estimates by applying the depth-diversity criterion after the distance-to-go estimate. For example, $\left[f^{\mathrm{LMcut}}, \hat{h}^{\mathrm{FF}},\langle d\rangle, f i f o\right]$ applies depth diversification criterion after the $\hat{h}^{\mathrm{FF}}$ distance-to-go estimate. As we shall see below, a sorting strategy which performs tie-breaking using both distance-to-go estimates and depth diversity results in the best performance overall.

\subsection{Evaluation of Distance-to-Go Estimates as Tie-Breaking Criteria for Admissible Search}

We tested various admissible sorting strategies on IPC domains and Zerocost domains. The configurations are listed in Table 8.1. In all configurations, the first sorting criterion is the $f=g+h$ value where $h$ is an admissible heuristic (either LMcut or M\&S) using the actual action-cost based cost calculation. As the second (and third) criteria, we used $\hat{h}$, the distance-to-go version tested of the original heuristic function $h$, as well as a distance-to-go variation of FF heuristic $\left(\hat{h}^{\mathrm{FF}}\right)$. We also added configurations with the depth metric within plateau $\left(f, \hat{h}^{\mathrm{FF}}\right)$. A summary of the results is shown in Table 8.2. Detailed per-domain results are shown in Tables A.7-A.10. 

(1) $[h+g$
(2) $[h+g, \quad h, \quad \hat{h}, \quad *$
(3) $[h+g, \quad \hat{h}, \quad *]$
(4) $\left[h+g, \quad \hat{h}^{\mathrm{FF}}, *\right]$
(5) $[h+g, \quad h,\langle d\rangle$,
(6) $\left[h+g, \quad \hat{h}^{\mathrm{FF}},\langle d\rangle, *\right]$

Table 8.1: Configurations compared in this section. $h$ is either LMcut or M\&S.

\begin{tabular}{|c|c|c|c|c|}
\hline Sorting Criteria & $\begin{array}{l}\text { Zerocost }(620) \\
h=\text { LMcut }\end{array}$ & $\begin{array}{l}\text { Zerocost }(620) \\
h=\mathrm{M} \& \mathrm{~S}\end{array}$ & $\begin{array}{l}\text { IPC (1104) } \\
h=\text { LMcut }\end{array}$ & $\begin{array}{l}\text { IPC (1104) } \\
h=\mathrm{M} \& \mathrm{~S}\end{array}$ \\
\hline Baselines & & & & \\
\hline$[f, h, f i f o]$ & 256 & 280 & 558 & 491 \\
\hline$[f, h$, lifo $]$ & 279 & 301 & 565 & 496 \\
\hline$[f, h, r o]$ & $261.9 \pm 1.4$ & $287.7 \pm 3.2$ & $558.9 \pm 2.1$ & $489.4 \pm 1.0$ \\
\hline$[f, h,\langle d\rangle, f i f o]$ & 284 & 302 & 571 & 487 \\
\hline$[f, h,\langle d\rangle$, lifo $]$ & 264 & 288 & 575 & 487 \\
\hline$[f, h,\langle d\rangle, r o]$ & $288.1 \pm 1.6$ & $308.1 \pm 2.1$ & $571.4 \pm 1.7$ & $485.6 \pm 1.5$ \\
\hline Distance-to-Go & & & & \\
\hline$[f, \hat{h}$, fifo $]$ & 295 & 308 & 534 & 477 \\
\hline$[f, \hat{h}$, lifo $]$ & 303 & 305 & 534 & 475 \\
\hline$[f, \hat{h}, r o]$ & 301.0 & $307.3 \pm 1.5$ & $534 \pm 2.1$ & $470.4 \pm 0.9$ \\
\hline$[f, h, \hat{h}, f i f o]$ & 305 & 307 & 536 & 476 \\
\hline$[f, h, \hat{h}$, lifo $]$ & 309 & 306 & 535 & 475 \\
\hline$[f, h, \hat{h}, r o]$ & $305.9 \pm 2.1$ & $307.8 \pm 1.4$ & $534.7 \pm 1.5$ & $470.9 \pm 0.9$ \\
\hline$\left[f, \hat{h}^{\mathrm{FF}}, f i f o\right]$ & 337 & 336 & 564 & 458 \\
\hline$\left[f, \hat{h}^{\mathrm{FF}}\right.$, lifo $]$ & 340 & 331 & 562 & 457 \\
\hline$\left[f, \hat{h}^{\mathrm{FF}}, r o\right]$ & $341 \pm 2.2$ & $337.9 \pm 2.1$ & $563.7 \pm 1.4$ & $457 \pm 1.3$ \\
\hline Distance + Depth & & & & \\
\hline$\left[f, \hat{h}^{\mathrm{FF}},\langle d\rangle, f f f o\right]$ & $\underline{340}(>337)$ & $\underline{337}(>336)$ & 563 & 457 \\
\hline$\left[f, \hat{h}^{\mathrm{FF}},\langle d\rangle\right.$, lifo $]$ & $\underline{342}(>340)$ & $\underline{333}(>331)$ & 560 & 457 \\
\hline$\left[f, \hat{h}^{\mathrm{FF}},\langle d\rangle, r o\right]$ & $\overline{344.3} \pm 1.8$ & $\overline{337.6} \pm 1.3$ & $561.9 \pm 1.4$ & $456.8 \pm 1.2$ \\
\hline
\end{tabular}

Table 8.2: Summary Results: Coverage comparison (the number of instances solved in 5min, 4GB) between several sorting strategies. For comparison, we also include the results of configurations evaluated in the previous sections. 


\subsubsection{Evaluation on Zerocost Domains}

In Zerocost domains, we see that $\hat{h}$ tie-breaking outperforms $h$ tie-breaking for both LMcut (e.g. $256 \rightarrow 295$ with fifo) and M\&S (e.g. $280 \rightarrow 308$ with fifo). Also, combining $h$ and $\hat{h}$ can further improve performance when the heuristic is LMcut (e.g. $295 \rightarrow 305$ with fifo). The results of combining $h$ and $\hat{h}$ were comparable to $\hat{h}$ when the main heuristic function $h$ is M\&S. Yet more surprisingly, using $\hat{h}^{\mathrm{FF}}$ further improved the performance for both LMcut (e.g. $[f, h, \hat{h}$, fifo $]: 305 \rightarrow$ $\left[f, \hat{h}^{\mathrm{FF}}\right.$, fifo $]: 337$ ) and M\&S (e.g. $[f, h, \hat{h}$, fifo $]: 307 \rightarrow\left[f, \hat{h}^{\mathrm{FF}}\right.$, fifo $\left.]: 336\right)$. Thus, when the depth diversity criterion is not used, the best configurations are those which use $\hat{h}^{\mathrm{FF}}$.

The reason for the good performance of $\left[f^{\mathrm{LMcut}}, \hat{h}^{\mathrm{FF}}, *\right]$ is not surprising: $\hat{h}^{\mathrm{FF}}$ is by itself known to be a powerful inadmissible heuristic function for satisficing GBFS, and if we ignore the first sorting criterion, $\left[f^{\mathrm{LMcut}}, \hat{h}^{\mathrm{FF}}, *\right]$ is a GBFS with $\left[\hat{h}^{\mathrm{FF}}, *\right]$.

Adding the depth diversity criterion further improves the performance of the $\hat{h}^{\mathrm{FF}}$-based strategies, although the impact was small. The coverage increased in both $h=h^{\text {LMcut }}$ (fifo: $337 \rightarrow 340$, lifo: $340 \rightarrow 342$, ro: $341 \rightarrow 344.3$ ) and $h=h^{\mathrm{M} \& \mathrm{~S}}$ (fifo: $336 \rightarrow 337$, lifo: $331 \rightarrow 333$ ). When the default tie-breaking was $r o$ and the heuristic is $\mathrm{M} \& \mathrm{~S},\left[f, \hat{h}^{\mathrm{FF}},\langle d\rangle, r o\right]$ performed slightly worse than $\left[f, \hat{h}^{\mathrm{FF}}\right.$, ro $]$, but the difference was very small $(337.9 \rightarrow 337.6)$ and $\langle d\rangle$ made the performance slightly more robust (smaller standard deviation: $2.1 \rightarrow 1.3$ ).

\subsubsection{Evaluation ON STANDARd IPC DOMAins}

For the standard IPC benchmark instances, the overhead due to the additional computation of $\hat{h}$ or $\hat{h}^{\mathrm{FF}}$ tends to harm the overall performance. Therefore, the best configuration using LMcut was $[f, h,\langle d\rangle$, lifo $]$ which uses depth and does not impose the cost of additional heuristics, and the best result using M\&S was $[f, h$, lifo $]$ which imposes no overhead including the depth.

If we look further into the detail, we observed the following: In Cybersec, distance-to-go variants (e.g. $\left[f^{\text {LMcut }}, \hat{h}^{\mathrm{FF}}\right.$, lifo $\left.]: 5\right)$ improve upon the standard strategy (e.g. $\left.\left[f^{\mathrm{LMcut}}, h^{\mathrm{LMcut}}, l i f o\right]: 3\right)$, but does not improve upon depth (e.g. $\left[f, h,\langle d\rangle\right.$, lifo]: 12). When $h=h^{\mathrm{M} \& \mathrm{~S}}$, all coverages are zero. Overheads by $\hat{h}^{\mathrm{FF}}$ also slightly degrade the performance in Openstacks (e.g. [ $f^{\text {LMcut }}, h^{\text {LMcut }}$, lifo]:18, $\left[f^{\mathrm{LMcut}}, \hat{h}^{\mathrm{FF}}, l\right.$ lifo $]: 17,\left[f^{\mathrm{LMcut}}, h^{\mathrm{LMcut}},\langle d\rangle\right.$, lifo $]: 18$; Also, $\left[f^{\mathrm{M} \& \mathrm{~S}}, h^{\mathrm{M} \& \mathrm{~S}}\right.$, lifo $]: 19,\left[f^{\mathrm{M} \& \mathrm{~S}}, \hat{h}^{\mathrm{FF}}\right.$, lifo $]: 18$, $\left[f^{\mathrm{M} \& \mathrm{~S}}, h^{\mathrm{M} \& \mathrm{~S}},\langle d\rangle\right.$, lifo $\left.]: 19\right)$. Thus, in these two domains, although there are some improvements in search efficiency due to the guidance by $\hat{h}^{\mathrm{FF}}$ or $\hat{h}$, the runtime overhead of computing the distanceto-go heuristics outweighed the benefit.

In the domains with only positive cost actions (all IPC domains except Openstacks and Cybersec), $\hat{h}$ or $\hat{h}^{\mathrm{FF}}$ only harm the overall performance due to the overhead. When the primary heuristics is LMcut, we do not observe a significant difference between single-heuristics strategies except for the fractional difference in the configurations using ro. When the primary heuristic is $\mathrm{M} \& \mathrm{~S}$, $\left[f^{\mathrm{M} \& \mathrm{~S}}, h^{\mathrm{M} \& \mathrm{~S}}\right.$, lifo $]$ performs slightly better than other default tie-breaking strategies; It also outperforms the depth-based variants as we already discussed in Section 6 .

\subsubsection{SUMMARY OF THE EVALUATION}

Table 8.3 summarizes the overall conclusions of our performance evaluations. We conclude that although the performance gain by depth diversification and distance-to-go heuristics depend on the domain characteristics, they provide a promising overall performance enhancement. 


\begin{tabular}{|l|l||l|l|}
\hline $\begin{array}{l}\text { Primary } \\
\text { Heuristics }\end{array}$ & Zerocost domains & $\begin{array}{l}\text { Zerocost IPC domains } \\
\text { (Cybersec, Openstacks) }\end{array}$ & $\begin{array}{l}\text { Positive-cost IPC do- } \\
\text { mains }\end{array}$ \\
\hline LMcut & {$\left[f, \hat{h}^{\mathrm{FF}},\langle d\rangle, r o\right]$} & {$[f, h,\langle d\rangle$, lifo $]$} & $\begin{array}{l}{[f, h, *] \text { or }[f, h,\langle d\rangle, *]} \\
\text { any default tie- } \\
\text { breaking) }\end{array}$ \\
\hline M\&S & $\begin{array}{l}{\left[f, \hat{h}^{\mathrm{FF}}, r o\right] \text { or }} \\
{\left[f, \hat{h}^{\mathrm{FF}},\langle d\rangle, r o\right],} \\
\text { but the latter has a } \\
\text { smaller variance. }\end{array}$ & $\begin{array}{l}{[f, h, \text { lifo }] \text { or }[f, h,\langle d\rangle, *]} \\
\text { (any default tie- } \\
\text { breaking) }\end{array}$ & {$[f, h$, lifo $]$} \\
\hline
\end{tabular}

Table 8.3: Summary of the performance evaluation: Best tie-breaking strategy for each group of domains and each primary heuristic function.

\subsection{Simple Dynamic Configuration for Overall Performance}

In practice, the performance degradation when using multi-heuristic strategy in domains with only positive cost actions does not pose a problem. We can easily avoid the overhead incurred by the distance-to-go heuristics in those domains by applying the following simple policy: If there are any 0-cost actions, use a multi-heuristic strategy; Otherwise, use a single-heuristic strategy.

Since the impact of such a check on the total runtime is negligible, we can extrapolate the result of applying this rule based on the previously obtained results. Coverage results in Table 8.4 show the total coverage of Zerocost and IPC benchmark domains. The bottom two rows, labeled as dynamic configuration, are the extrapolated results when the switching policy is applied - this dynamic configuration achieves the highest overall coverage.

When the configuration rule is applied to standard IPC instances, the domains with 0-cost actions are Cybersec and Openstacks only. They are solved using a multi-heuristic strategy while other domains are solved in the best performing single-heuristic strategy. In Zerocost instances, all domains are solved using the multi-heuristic strategy.

Overall, these results also strengthen our claim that one should not necessarily rely upon $h$-based tie-breaking in some domains, as already discussed in Section 3.1. In Zerocost domains, using a distance-to-go version of an inadmissible heuristic function for tie-breaking is more effective. Also, combining the depth metric with such an inadmissible heuristics is also effective.

We only tested this relatively simple dynamic configuration that switches between two strategies based on the presence of 0-cost operators. However, as noted in Section 6, domain-specific solvers (as opposed to domain-independent solvers, which are the main focus of this paper) can benefit from fine-tuning the tiebreaking strategy so that it is most suited to the target domain.

\section{Related Work}

Previous work on escaping search space plateaus has focused on non-admissible search. DBFS (Imai \& Kishimoto, 2011) adds stochastic backtracking to Greedy Best First Search (GBFS) to avoid being misdirected by the heuristic function. Type based buckets (Xie et al., 2014) classify plateaus in GBFS according to the $[g, h]$ pair and distributes the effort $\left.\right|^{4}$ Marvin (Coles \& Smith,

4. The relationship between Type-GBFS and our work is discussed in detail in Section 7.1 


\begin{tabular}{|l|l|l|}
\hline & $\begin{array}{l}\text { IPC+Zerocost (1724) } \\
h=\text { LMcut }\end{array}$ & $\begin{array}{l}\text { IPC+Zerocost (1724) } \\
h=\text { M\&S }\end{array}$ \\
Single-heuristic strategies & 844 & 797 \\
{$[f, h$, lifo $]$} & 855 & 789 \\
{$[f, h,\langle d\rangle, f i f o]$} & 839 & 775 \\
{$[f, h,\langle d\rangle$, lifo $]$} & 859.5 & 793.7 \\
{$[f, h,\langle d\rangle, r o]$} & & \\
Multi-heuristic strategies & 903 & 794 \\
{$\left[f, \hat{h}^{\mathrm{FF}},\langle d\rangle, f i f o\right]$} & 790 \\
{$\left[f, \hat{h}^{\mathrm{FF}},\langle d\rangle, l i f o\right]$} & 794.4 \\
{$\left[f, \hat{h}^{\mathrm{FF}},\langle d\rangle, r o\right]$} & \\
Dynamic Configuration & 902 & \\
If a problem contains zerocost actions: & & \\
Then $\left[f, \hat{h}^{\mathrm{FF}},\langle d\rangle\right.$, ro $] ;$ Else $[f, h,\langle d\rangle$, lifo $]$ & $\mathbf{9 1 1 . 9}$ & $\mathbf{8 3 2 . 3}$ \\
If a problem contains zerocost actions: & & \\
Then $\left[f, \hat{h}^{\mathrm{FF}},\langle d\rangle\right.$, ro $] ;$ Else $[f, h$, lifo $]$ & & \\
\hline
\end{tabular}

Table 8.4: Summary Results: Coverage comparison, the total of IPC domains and Zerocost domains (the number of instances solved in $5 \mathrm{~min}, 4 \mathrm{~GB}$ ) between several sorting strategies, plus a dynamic configuration strategy. $[f, h, f i f o],[f, h, r o],[f, \hat{h}, *],[f, h, \hat{h}, *],\left[f, \hat{h}^{\mathrm{FF}}, *\right]$ are not shown because they achieve smaller coverage.

2007) learns plateau-escaping macros from the Enhanced Hill Climbing phase of the FF planner (Hoffmann \& Nebel, 2001). Hoffmann gives a detailed analysis of the structure of the search spaces of satisficing planning $(2005,2011)$.

Benton et al. (2010) proposes inadmissible technique for temporal planning where short actions are hidden behind long actions and do not increase makespan. Wilt and Ruml (2011) also analyzes inadmissible distance-to-go estimates. To our knowledge, plateaus have not been previously investigated for cost-optimal search. Admissible and inadmissible search differ significantly in how non-final plateaus (plateaus with $f<f^{*}$ ) are treated: Inadmissible search can skip or escape plateaus whenever possible, while admissible search cannot, unless it is the plateau with $f=f^{*}$ where the goals can immediately be found.

Some real-time search algorithms like $A R A^{*}$ (Likhachev, Ferguson, Gordon, Stentz, \& Thrun, 2008) are able to prune some states in the final plateau using the knowledge acquired in the previous iterations of suboptimal searches. $A R A^{*}$ uses a sequence of $W A^{*}([g+w h])$ with decreasing weights $w$, with the final round of iterations being optimal $A^{*}$ with an uninflated heuristic value (i.e. $w=1$ ). When $f=g+w h$ reaches the cost of best path found so far by the previous suboptimal iterations, it can safely terminate the search maintaining the current bounded optimality guarantee $w$, that is, $w=1$ in the final iteration. Thus, in an iterated, real-time search setting, this could largely avoid the problem of searching the final plateau if the previous suboptimal searches happen to have found the optimal solution already. 
In their work on combining multiple inadmissible heuristics in a planner, Röger and Helmert (2010) considered a tie-breaking approach which works as follows: When combining two heuristics $h_{1}$ and $h_{2}, h_{1}$ is used as the primary criterion, and $h_{2}$ is used to break ties among nodes with the same $h_{1}-\left[h_{1}, h_{2}\right.$, fifo $]$. This did not perform well in their work on satisficing planning compared to the approaches based on alternation queues and Pareto-optimal queue selection. Since their focus is on how to combine multiple heuristics, this tie-breaking-based approach is just one instance of various implementations of OPEN lists. In contrast, this paper provides a focused, in-depth investigation of various tie-breaking strategies, and shows how tie-breaking enables the efficient search on the plateau created by the earlier levels of sorting criteria.

$A^{*}$ with lookahead $\left(A L^{*}\right)$ (Stern, Kulberis, Felner, \& Holte, 2010) extends $A^{*}$ by performing a cost-bounded depth-first lookahead from each node as it is generated. Upon the normal expansion of a node $n$ in $A^{*}$, lookahead search performs a depth-first search with cost bound $f(n)+k$ rooted at $n$. As a special case, under the cost bound $k=0$ ( $A L_{0}^{*}$ in their notation), depth-first lookahead expands only the children with the same $f$-value. $A L^{*}$, or $A L_{0}^{*}$ in particular, is similar to $[f$, lifo $]$ in that the lookahead is a depth-first search. However, there are both conceptual and algorithmic differences: First of all, $A L_{0}^{*}$ does not specify the intermediate tie-breaking (such as $h$-based tiebreaking) for its main $A^{*}$, and depth-first lookahead does not perform best-first expansion, so the tie-breaking is irrelevant. Thus, the problems and the solutions addressed in these approaches are different. Second, $A L^{*}$ propagates the maximum and the minimum $f$ values found in the lookahead search, which allows for more pruning.

Another relevant line of work, in similar spirit to Zerocost domains, is the Preference Track in the deterministic part of IPC4 (Gerevini, Saetti, \& Vallati, 2009). One difference between our Zerocost domains and these domains is that the latter allows a more complex semantics such as multiplication. More recently, Wray et al. (2015) proposed a model called conditional lexicographic preferences with slack in the context of planning under uncertainty. Lexicographic preferences allow the problem to have multiple preference criteria evaluated individually. The solution quality is determined by the first preference, breaking ties by the second preference and so on. Slack refers to a constant amount of error from the optimal value. With slack, one can model a situation where the goal is to optimize the first preference, but the difference up to some amount is ignored and ties are broken according to the second preference. An example of a planning problem with such lexicographic preferences with slack would be a transportation problem where the first optimization objective is the amount of fuel usage, allowing a slack up to 5 liters, and the second optimization target is the makespan of the plan. In this case, a plan with 100 liters of fuel usage and a plan with 105 liters of fuel usage are considered equally preferable in the first criterion, and the better plan is the one with a shorter makespan. Since slack allows multiple values (e.g. 100 and 105) to have the same preference, it should introduce larger plateaus. Applying our techniques to problems with slack is an avenue of future work.

\section{Conclusions and Future Work}

In this paper, we investigated tie-breaking strategies for cost-optimal search using $A^{*}$. Our contributions are as follows: First, we showed that tie-breaking has a significant role in the cost-optimal search using $A^{*}$. We empirically showed that most IPC benchmark instances have large plateaus with regard to $f$, and most of the search effort is spent in the final plateau with $f=f^{*}$. 
We then showed that the commonly used tie-breaking policy based on $h$ value fails to provide guidance in the plateau when problem instances have 0-cost actions and have large plateaus with regard to $h$. We empirically showed that most of the search effort can be spent in the final plateau with $f=f^{*}, h=0$ in some domains, and noted that in such a plateau, the search is controlled solely by the default tie-breaking fifo, lifo or ro.

We proposed a new set of benchmark instances for cost-optimal planning, called Zerocost domains, which contain many 0 -cost actions. We showed that Zerocost versions of IPC benchmark domains tend to have larger final plateaus with $f=f^{*}, h=0$ and pose a new challenge to traditional search algorithms.

As one approach to improving search performance in Zerocost domains, we proposed a depth metric which measures the distance from the entrance to the plateau. Using this metric, we described the pathological behaviors of fifo, lifo and ro, proposed a new diversification strategy, theoretically and empirically showed that it avoids the pathological behavior and achieves a better performance.

We then introduced a new interpretation of cost-optimal $A^{*}$ search as a series of satisficing searches among $f$-cost plateaus of an increasing order of $f$. This perspective led to another approach for effective tie-breaking in Zerocost domains, the use of inadmissible distance-to-go estimates as part of a multi-heuristics tie-breaking strategy. Combination of depth diversification and distance-to-go estimates results in the best overall performance. Although there is an additional cost to compute multiple heuristic values, the overhead can be eliminated by a simple case-based configuration which only uses multiple heuristics when 0 -cost actions are present in the problem instance.

Our reformulation of $A^{*}$ as a sequence of satisficing searches points to an interesting direction for future work. Although we evaluated only one relatively simple, satisficing configuration $\left(\hat{h}^{\mathrm{FF}}\right)$ in the experiments, many techniques which have previously been developed for satisficing planning can be applied to enhance tie-breaking (plateau-search) in cost-optimal search, including lazy evaluation (Richter \& Westphal, 2010), alternating/Pareto open list (Röger \& Helmert, 2010), helpful actions (preferred operators) (Hoffmann \& Nebel, 2001), random walk local search (Nakhost \& Müller, 2009), macro operators (Botea, Enzenberger, Müller, \& Schaeffer, 2005; Chrpa, Vallati, \& McCluskey, 2015), factored planning (Amir \& Engelhardt, 2003; Brafman \& Domshlak, 2006; Asai \& Fukunaga, 2015) and exploration-based search enhancements (Valenzano et al., 2014; Xie et al., 2014; Valenzano \& Xie, 2016).

\section{Acknowledgments}

Thanks to the anonymous reviewers for numerous helpful suggestions which significantly improved the paper. This research was supported by a JSPS Grant-in-Aid for JSPS Fellows and a JSPS KAKENHI grant. 


\section{Appendix A. Detailed Data}

This Appendix contains some detailed figures and data which are referenced from the text in the previous sections.

\section{A.1 Detailed Data for Table 3.1}

\begin{tabular}{|c|c|c|c|c|c|c|}
\hline Domain & {$[f, f i f o]$} & {$[f$, lifo $]$} & {$[f, r o]$} & {$[f, h, f i f o]$} & {$[f, h$, lifo $]$} & {$[f, h, r o]$} \\
\hline IPC benchmark (1104) & 443 & 558 & $448.9 \pm 1.3$ & 558 & 565 & $558.9 \pm 2.1$ \\
\hline airport(50) & 18 & 26 & $18 \pm 0$ & 27 & 26 & $25.7 \pm 0.5$ \\
\hline barman-opt11(20) & 0 & 0 & $0 \pm 0$ & 0 & 0 & $0 \pm 0$ \\
\hline blocks (35) & 26 & 26 & $26 \pm 0$ & 28 & 28 & $\mathbf{2 8} \pm 0$ \\
\hline cybersec(19) & 0 & 3 & $0 \pm 0$ & 2 & 3 & $3.9 \pm 1.1$ \\
\hline $\operatorname{depot}(22)$ & 5 & 5 & $5 \pm 0$ & 6 & 6 & $6 \pm 0$ \\
\hline driverlog $(20)$ & 12 & 13 & $12 \pm 0$ & 13 & 13 & $13 \pm 0$ \\
\hline elevators-opt11(20) & 14 & 15 & $14 \pm 0$ & 15 & 15 & $15 \pm 0$ \\
\hline floortile-opt11(20) & 6 & 6 & $6 \pm 0$ & 6 & 6 & $6 \pm 0$ \\
\hline freecell(80) & 8 & 9 & $8.7 \pm 0.5$ & 9 & 9 & $9 \pm 0$ \\
\hline $\operatorname{grid}(5)$ & 1 & 1 & $1 \pm 0$ & 1 & 1 & $1 \pm 0$ \\
\hline gripper(20) & 6 & 6 & $6 \pm 0$ & 6 & 6 & $6 \pm 0$ \\
\hline hanoi(30) & 12 & 12 & $12 \pm 0$ & 12 & 12 & $12 \pm 0$ \\
\hline logistics00(28) & 16 & 18 & $16 \pm 0$ & 20 & 20 & $\mathbf{2 0} \pm 0$ \\
\hline miconic(150) & 68 & 140 & $68 \pm 0$ & 140 & 140 & $140 \pm 0$ \\
\hline mprime(35) & 20 & 22 & $19.9 \pm 0.3$ & 21 & 21 & $20.9 \pm 0.3$ \\
\hline mystery(30) & 15 & 16 & $15 \pm 0$ & 16 & 16 & $15.2 \pm 0.4$ \\
\hline nomystery-opt11(20) & 12 & 13 & $12 \pm 0$ & 14 & 14 & $\mathbf{1 4} \pm 0$ \\
\hline openstacks-opt11(20) & 11 & 18 & $11.2 \pm 0.4$ & 11 & 18 & $11.7 \pm 0.5$ \\
\hline parcprinter-opt11(20) & 12 & 13 & $12 \pm 0$ & 13 & 13 & $13 \pm 0$ \\
\hline parking-opt11(20) & 1 & 1 & $1 \pm 0$ & 1 & 1 & $1 \pm 0$ \\
\hline pathways(30) & 4 & 5 & $4 \pm 0$ & 5 & 5 & $5 \pm 0$ \\
\hline pegsol-opt11(20) & 17 & 17 & $17 \pm 0$ & 17 & 17 & $17 \pm 0$ \\
\hline pipesworld-notankage(50) & 13 & 13 & $13 \pm 0$ & 14 & 14 & $14.6 \pm 0.5$ \\
\hline pipesworld-tankage(50) & 7 & 8 & $8 \pm 0$ & 8 & 8 & $8 \pm 0$ \\
\hline psr-small(50) & 48 & 48 & $48 \pm 0$ & 48 & 48 & $48 \pm 0$ \\
\hline rovers $(40)$ & 7 & 7 & $7 \pm 0$ & 7 & 7 & $7 \pm 0$ \\
\hline scanalyzer-opt11(20) & 4 & 10 & $5.4 \pm 0.7$ & 10 & 10 & $\mathbf{1 0} \pm 0$ \\
\hline sokoban-opt11(20) & 19 & 19 & $19 \pm 0$ & 19 & 19 & $19 \pm 0$ \\
\hline storage(30) & 14 & 14 & $14 \pm 0$ & 14 & 14 & $14 \pm 0$ \\
\hline tidybot-opt11(20) & 11 & 12 & $11 \pm 0$ & 12 & 12 & $12 \pm 0$ \\
\hline $\operatorname{tpp}(30)$ & 6 & 6 & $6 \pm 0$ & 6 & 6 & $6 \pm 0$ \\
\hline transport-opt11(20) & 6 & 6 & $6 \pm 0$ & 6 & 6 & $6 \pm 0$ \\
\hline visitall-opt11(20) & 9 & 10 & $9.4 \pm 0.5$ & 10 & 10 & $10 \pm 0$ \\
\hline woodworking-opt11(20) & 6 & 9 & $8.2 \pm 0.4$ & 10 & 10 & $\mathbf{1 0} \pm 0$ \\
\hline zenotravel(20) & 9 & 11 & $9 \pm 0$ & 11 & 11 & $\mathbf{1 1} \pm 0$ \\
\hline
\end{tabular}

Table A.1: Coverage comparison (the number of instances solved in 5min, 4GB, LMcut heuristics) among the standard baseline tie-breaking algorithms. We highlight the best results when the difference between the maximum and the minimum coverage exceeds 2 . 


\begin{tabular}{|c|c|c|c|c|c|c|}
\hline Domain & {$[f, f i f o]$} & {$[f$, lifo $]$} & {$[f, r o]$} & {$[f, h, f i f o]$} & {$[f, h$, lifo $]$} & {$[f, h, r o]$} \\
\hline IPC benchmark (1104) & 460 & 490 & $460.9 \pm 1.6$ & 491 & 496 & $489.4 \pm 1.0$ \\
\hline airport(50) & 9 & 9 & $9 \pm 0$ & 9 & 9 & $9 \pm 0$ \\
\hline barman-opt11(20) & 4 & 4 & $4 \pm 0$ & 4 & 4 & $4 \pm 0$ \\
\hline blocks(35) & 21 & 22 & $21 \pm 0$ & 22 & 22 & $22 \pm 0$ \\
\hline cybersec(19) & 0 & 0 & $0 \pm 0$ & 0 & 0 & $0 \pm 0$ \\
\hline $\operatorname{depot}(22)$ & 5 & 6 & $5 \pm 0$ & 6 & 6 & $5 \pm 0$ \\
\hline driverlog $(20)$ & 12 & 12 & $12 \pm 0$ & 12 & 12 & $12 \pm 0$ \\
\hline elevators-opt11(20) & 13 & 13 & $13 \pm 0$ & 13 & 13 & $13 \pm 0$ \\
\hline floortile-opt11(20) & 5 & 6 & $5 \pm 0$ & 6 & 6 & $6 \pm 0$ \\
\hline freecell(80) & 15 & 16 & $15 \pm 0$ & 17 & 17 & $16 \pm 0$ \\
\hline $\operatorname{grid}(5)$ & 2 & 2 & $2 \pm 0$ & 2 & 2 & $2 \pm 0$ \\
\hline gripper(20) & 8 & 20 & $8 \pm 0$ & 20 & 20 & $\mathbf{2 0} \pm 0$ \\
\hline hanoi(30) & 14 & 14 & $14 \pm 0$ & 14 & 14 & $14 \pm 0$ \\
\hline logistics00(28) & 20 & 20 & $20 \pm 0$ & 20 & 20 & $20 \pm 0$ \\
\hline miconic $(150)$ & 68 & 73 & $68.3 \pm 0.7$ & 73 & 73 & $73.2 \pm 0.4$ \\
\hline mprime(35) & 23 & 23 & $22 \pm 0$ & 23 & 24 & $23.7 \pm 0.5$ \\
\hline mystery(30) & 15 & 15 & $15 \pm 0$ & 15 & 16 & $15 \pm 0$ \\
\hline nomystery-opt11(20) & 17 & 18 & $17.8 \pm 0.4$ & 18 & 18 & $18 \pm 0$ \\
\hline openstacks-opt11(20) & 15 & 19 & $15.4 \pm 0.5$ & 15 & 19 & $15.4 \pm 0.5$ \\
\hline parcprinter-opt11(20) & 10 & 10 & $10 \pm 0$ & 10 & 10 & $10 \pm 0$ \\
\hline parking-opt11(20) & 1 & 1 & $1 \pm 0$ & 1 & 1 & $1 \pm 0$ \\
\hline pathways(30) & 4 & 4 & $4 \pm 0$ & 4 & 4 & $4 \pm 0$ \\
\hline pegsol-opt11(20) & 17 & 19 & $17.2 \pm 0.4$ & 19 & 19 & $\mathbf{1 9} \pm 0$ \\
\hline pipesworld-notankage(50) & 9 & 9 & $8.9 \pm 0.3$ & 10 & 10 & $9.9 \pm 0.3$ \\
\hline pipesworld-tankage(50) & 13 & 13 & $13.1 \pm 0.3$ & 13 & 13 & $13.2 \pm 0.4$ \\
\hline psr-small(50) & 50 & 50 & $50 \pm 0$ & 50 & 50 & $50 \pm 0$ \\
\hline rovers $(40)$ & 6 & 8 & $6.1 \pm 0.3$ & 8 & 8 & $8 \pm 0$ \\
\hline scanalyzer-opt11(20) & 10 & 10 & $10 \pm 0$ & 10 & 10 & $10 \pm 0$ \\
\hline sokoban-opt11(20) & 20 & 20 & $20 \pm 0$ & 20 & 20 & $20 \pm 0$ \\
\hline storage(30) & 15 & 15 & $15 \pm 0$ & 15 & 15 & $15 \pm 0$ \\
\hline tidybot-opt11(20) & 0 & 0 & $0 \pm 0$ & 0 & 0 & $0 \pm 0$ \\
\hline $\operatorname{tpp}(30)$ & 6 & 6 & $6 \pm 0$ & 7 & 6 & $6 \pm 0$ \\
\hline transport-opt11(20) & 7 & 7 & $7 \pm 0$ & 7 & 7 & $7 \pm 0$ \\
\hline visitall-opt11(20) & 9 & 9 & $9 \pm 0$ & 9 & 9 & $9 \pm 0$ \\
\hline woodworking-opt11(20) & 7 & 7 & $7 \pm 0$ & 7 & 7 & $7 \pm 0$ \\
\hline zenotravel(20) & 10 & 10 & $10 \pm 0$ & 12 & 12 & $\mathbf{1 2} \pm 0$ \\
\hline
\end{tabular}

Table A.2: Coverage comparison (the number of instances solved in 5min, 4GB, M\&S heuristics) among the standard baseline tie-breaking algorithms. We highlight the best results when the difference between the maximum and the minimum coverage exceeds 2 . 


\section{A.2 Detailed Data for Table 6.1}

\begin{tabular}{|c|c|c|c|c|c|c|}
\hline Zerocost (620) & 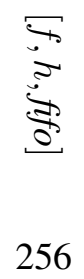 &  & $261.9 \pm 1.4$ & 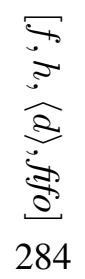 & 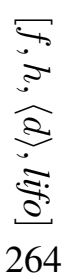 & 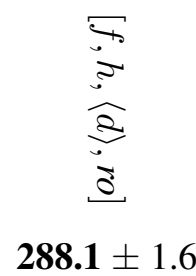 \\
\hline airport-fuel(20) & 15 & 13 & $13.8 \pm 0.4$ & 14 & 13 & $14 \pm 0.5$ \\
\hline blocks-stack(20) & 17 & 17 & $17 \pm 0$ & 17 & 17 & $17 \pm 0$ \\
\hline depot-fuel(22) & 6 & 6 & $6 \pm 0$ & 6 & 6 & $6 \pm 0$ \\
\hline driverlog-fuel(20) & 8 & 8 & $8 \pm 0$ & 8 & 8 & $8 \pm 0$ \\
\hline elevators-up(20) & 7 & 13 & $7 \pm 0$ & 7 & 9 & $9.1 \pm 0.8$ \\
\hline floortile-ink(20) & 8 & 8 & $8.1 \pm 0.3$ & 8 & 8 & $8.2 \pm 0.4$ \\
\hline freecell-move $(20)$ & 4 & 19 & $4.9 \pm 0.3$ & 17 & 10 & $16.4 \pm 0.7$ \\
\hline grid-fuel(5) & 1 & 1 & $1 \pm 0$ & 1 & 1 & $1 \pm 0$ \\
\hline gripper-move(20) & 7 & 7 & $7 \pm 0$ & 7 & 7 & $7 \pm 0$ \\
\hline hiking-fuel(20) & 9 & 9 & $9 \pm 0$ & 9 & 9 & $9 \pm 0$ \\
\hline logistics00-fuel(28) & 16 & 16 & $16 \pm 0$ & 16 & 16 & $15.3 \pm 0.5$ \\
\hline miconic-up(30) & 16 & 17 & $16.6 \pm 0.5$ & 19 & 18 & $\mathbf{2 0 . 3} \pm 0.7$ \\
\hline mprime-succumb (35) & 15 & 14 & $17.1 \pm 0.8$ & 22 & 14 & $20.1 \pm 0.3$ \\
\hline mystery-feast(20) & 7 & 5 & $7.7 \pm 0.5$ & 6 & 5 & $7.2 \pm 0.8$ \\
\hline nomystery-fuel(20) & 10 & 10 & $10 \pm 0$ & 10 & 10 & $10 \pm 0$ \\
\hline parking-movecc(20) & 0 & 0 & $0 \pm 0$ & 0 & 0 & $0 \pm 0$ \\
\hline pathways-fuel(30) & 5 & 5 & $4.3 \pm 0.5$ & 5 & 5 & $4.1 \pm 0.3$ \\
\hline pipesnt-pushstart (20) & 8 & 8 & $8.4 \pm 0.5$ & 8 & 8 & $\mathbf{9 . 8} \pm 0.4$ \\
\hline pipesworld-pushend(20) & 3 & 4 & $3.8 \pm 0.4$ & 3 & 3 & $\mathbf{4 . 8} \pm 0.4$ \\
\hline psr-small-open (20) & 19 & 19 & $19 \pm 0$ & 19 & 19 & $19 \pm 0$ \\
\hline rovers-fuel(40) & 8 & 8 & $8 \pm 0$ & 8 & 8 & $8 \pm 0$ \\
\hline scanalyzer-analyze (20) & 9 & 9 & $9.1 \pm 0.3$ & 9 & 10 & $9.2 \pm 0.4$ \\
\hline sokoban-pushgoal(20) & 18 & 18 & $18 \pm 0$ & 18 & 18 & $18 \pm 0$ \\
\hline storage-lift(20) & 4 & 4 & $4.1 \pm 0.3$ & 5 & 4 & $4.2 \pm 0.4$ \\
\hline tidybot-motion(20) & 16 & 16 & $16 \pm 0$ & 16 & 16 & $16 \pm 0$ \\
\hline tpp-fuel(30) & 8 & 11 & $8 \pm 0$ & 11 & 10 & $\mathbf{1 1} \pm 0$ \\
\hline woodworking-cut $(20)$ & 5 & 7 & $7 \pm 0$ & 8 & 5 & $\mathbf{8 . 2} \pm 0.8$ \\
\hline zenotravel-fuel(20) & 7 & 7 & $7 \pm 0$ & 7 & 7 & $7 \pm 0$ \\
\hline
\end{tabular}

Table A.3: Coverage comparison (the number of instances solved in 5min, 4GB, LMcut heuristics) on 620 Zerocost instances. We highlight the best results when the difference between the best and the worst coverages is greater than 2 . 


\begin{tabular}{|c|c|c|c|c|c|c|}
\hline Zerocost (620) & 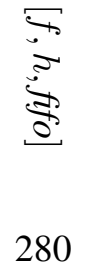 & 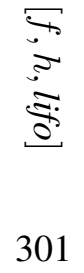 & $287.7 \pm 3.2$ & 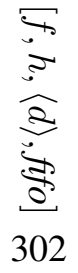 & 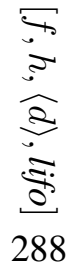 & $\begin{array}{c}\text { के } \\
\text { a } \\
\text { a } \\
\vdots \\
308.1 \pm 2.1\end{array}$ \\
\hline airport-fuel(20) & 5 & 5 & $5 \pm 0$ & 5 & 5 & $5 \pm 0$ \\
\hline blocks-stack(20) & 20 & 20 & $20 \pm 0$ & 20 & 20 & $20 \pm 0$ \\
\hline depot-fuel(22) & 5 & 5 & $6 \pm 0$ & 6 & 5 & $6 \pm 0$ \\
\hline driverlog-fuel(20) & 9 & 9 & $9 \pm 0$ & 9 & 9 & $9 \pm 0$ \\
\hline elevators-up(20) & 8 & 14 & $8.6 \pm 0.5$ & 9 & 13 & $11 \pm 1$ \\
\hline floortile-ink(20) & 8 & 8 & $8 \pm 0$ & 7 & 7 & $6.9 \pm 0.3$ \\
\hline freecell-move(20) & 5 & 17 & $6.7 \pm 0.9$ & 17 & 15 & $\mathbf{1 7 . 3} \pm 0.5$ \\
\hline grid-fuel(5) & 2 & 2 & $2 \pm 0$ & 2 & 2 & $2 \pm 0$ \\
\hline gripper-move(20) & 20 & 20 & $20 \pm 0$ & 20 & 20 & $20 \pm 0$ \\
\hline hiking-fuel(20) & 13 & 13 & $12.8 \pm 0.4$ & 13 & 12 & $12.1 \pm 0.3$ \\
\hline logistics00-fuel(28) & 16 & 16 & $16 \pm 0$ & 16 & 16 & $16 \pm 0$ \\
\hline miconic-up(30) & 29 & 30 & $30 \pm 0$ & 30 & 30 & $30 \pm 0$ \\
\hline mprime-succumb(35) & 21 & 19 & $19.6 \pm 0.7$ & 25 & 15 & $23.4 \pm 0.9$ \\
\hline mystery-feast(20) & 4 & 4 & $5.9 \pm 0.3$ & 4 & 4 & $6 \pm 0$ \\
\hline nomystery-fuel(20) & 16 & 16 & $16 \pm 0$ & 16 & 16 & $16 \pm 0$ \\
\hline parking-movecc(20) & 0 & 0 & $0 \pm 0$ & 0 & 0 & $0 \pm 0$ \\
\hline pathways-fuel(30) & 4 & 4 & $4 \pm 0$ & 4 & 4 & $4 \pm 0$ \\
\hline pipesnt-pushstart(20) & 3 & 3 & $3.4 \pm 0.5$ & 5 & 3 & $\mathbf{5} \pm 0$ \\
\hline pipesworld-pushend(20) & 5 & 9 & $7.7 \pm 0.5$ & 5 & 6 & $\mathbf{9} \pm 0.9$ \\
\hline psr-small-open(20) & 19 & 19 & $19 \pm 0$ & 19 & 19 & $19 \pm 0$ \\
\hline rovers-fuel(40) & 8 & 8 & $8 \pm 0$ & 8 & 8 & $8 \pm 0$ \\
\hline scanalyzer-analyze(20) & 11 & 11 & $11 \pm 0$ & 11 & 11 & $11 \pm 0$ \\
\hline sokoban-pushgoal(20) & 19 & 19 & $18 \pm 0$ & 18 & 18 & $18 \pm 0$ \\
\hline storage-lift(20) & 4 & 4 & $4 \pm 0$ & 4 & 4 & $4 \pm 0$ \\
\hline tidybot-motion(20) & 0 & 0 & $0 \pm 0$ & 0 & 0 & $0 \pm 0$ \\
\hline tpp-fuel(30) & 9 & 10 & $9.6 \pm 0.5$ & 11 & 10 & $\mathbf{1 1} \pm 0$ \\
\hline woodworking-cut(20) & 7 & 7 & $8 \pm 0.5$ & 8 & 7 & $9 \pm 1$ \\
\hline zenotravel-fuel(20) & 10 & 9 & $9.6 \pm 0.7$ & 10 & 9 & $9.3 \pm 1.0$ \\
\hline
\end{tabular}

Table A.4: Coverage comparison (the number of instances solved in 5min, 4GB, M\&S heuristics) on 620 Zerocost instances. We highlight the best results when the difference between the maximum and the minimum coverage exceeds 2 . 


\begin{tabular}{|c|c|c|c|c|c|c|}
\hline & 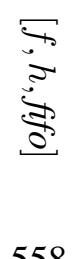 & 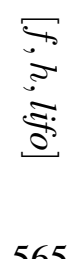 & $\begin{array}{l}5 \\
5 \\
5 \\
5 \\
5\end{array}$ & 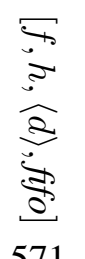 &  & 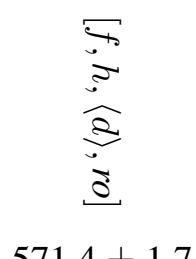 \\
\hline IPC benchmark (1104) & 558 & 565 & $558.9 \pm 2.1$ & & & \\
\hline airport(50) & 27 & 26 & $25.7 \pm 0.5$ & 27 & 26 & $25.7 \pm 0.5$ \\
\hline barman-opt11(20) & 0 & 0 & $0 \pm 0$ & 0 & 0 & $0 \pm 0$ \\
\hline $\operatorname{blocks}(35)$ & 28 & 28 & $28 \pm 0$ & 28 & 28 & $28 \pm 0$ \\
\hline cybersec(19) & 2 & 3 & $3.9 \pm 1.1$ & 8 & 12 & $10 \pm 1$ \\
\hline $\operatorname{depot}(22)$ & 6 & 6 & $6 \pm 0$ & 6 & 6 & $6 \pm 0$ \\
\hline driverlog $(20)$ & 13 & 13 & $13 \pm 0$ & 13 & 13 & $13 \pm 0$ \\
\hline elevators-opt11(20) & 15 & 15 & $15 \pm 0$ & 15 & 15 & $15 \pm 0$ \\
\hline floortile-opt11(20) & 6 & 6 & $6 \pm 0$ & 6 & 6 & $6 \pm 0$ \\
\hline freecell(80) & 9 & 9 & $9 \pm 0$ & 9 & 9 & $9 \pm 0$ \\
\hline $\operatorname{grid}(5)$ & 1 & 1 & $1 \pm 0$ & 1 & 1 & $1 \pm 0$ \\
\hline gripper(20) & 6 & 6 & $6 \pm 0$ & 6 & 6 & $6 \pm 0$ \\
\hline hanoi(30) & 12 & 12 & $12 \pm 0$ & 12 & 12 & $12 \pm 0$ \\
\hline logistics $00(28)$ & 20 & 20 & $20 \pm 0$ & 20 & 20 & $20 \pm 0$ \\
\hline miconic $(150)$ & 140 & 140 & $140 \pm 0$ & 140 & 140 & $140 \pm 0$ \\
\hline mprime(35) & 21 & 21 & $20.9 \pm 0.3$ & 21 & 21 & $20.9 \pm 0.3$ \\
\hline mystery(30) & 16 & 16 & $15.2 \pm 0.4$ & 16 & 16 & $15.4 \pm 0.5$ \\
\hline nomystery-opt11(20) & 14 & 14 & $14 \pm 0$ & 14 & 14 & $14 \pm 0$ \\
\hline openstacks-opt11(20) & 11 & 18 & $11.7 \pm 0.5$ & 18 & 18 & $\mathbf{1 8} \pm 0$ \\
\hline parcprinter-opt11(20) & 13 & 13 & $13 \pm 0$ & 13 & 13 & $13 \pm 0$ \\
\hline parking-opt11(20) & 1 & 1 & $1 \pm 0$ & 1 & 1 & $1 \pm 0$ \\
\hline pathways(30) & 5 & 5 & $5 \pm 0$ & 5 & 5 & $5 \pm 0$ \\
\hline pegsol-opt11(20) & 17 & 17 & $17 \pm 0$ & 17 & 17 & $17 \pm 0$ \\
\hline pipesworld-notankage(50) & 14 & 14 & $14.6 \pm 0.5$ & 14 & 15 & $14.4 \pm 0.5$ \\
\hline pipesworld-tankage(50) & 8 & 8 & $8 \pm 0$ & 8 & 8 & $8 \pm 0$ \\
\hline psr-small(50) & 48 & 48 & $48 \pm 0$ & 48 & 48 & $48 \pm 0$ \\
\hline rovers $(40)$ & 7 & 7 & $7 \pm 0$ & 7 & 7 & $7 \pm 0$ \\
\hline scanalyzer-opt11(20) & 10 & 10 & $10 \pm 0$ & 10 & 10 & $10 \pm 0$ \\
\hline sokoban-opt11(20) & 19 & 19 & $19 \pm 0$ & 19 & 19 & $19 \pm 0$ \\
\hline storage (30) & 14 & 14 & $14 \pm 0$ & 14 & 14 & $14 \pm 0$ \\
\hline tidybot-opt11(20) & 12 & 12 & $12 \pm 0$ & 12 & 12 & $12 \pm 0$ \\
\hline $\operatorname{tpp}(30)$ & 6 & 6 & $6 \pm 0$ & 6 & 6 & $6 \pm 0$ \\
\hline transport-opt11(20) & 6 & 6 & $6 \pm 0$ & 6 & 6 & $6 \pm 0$ \\
\hline visitall-opt11(20) & 10 & 10 & $10 \pm 0$ & 10 & 10 & $10 \pm 0$ \\
\hline woodworking-opt11(20) & 10 & 10 & $10 \pm 0$ & 10 & 10 & $10 \pm 0$ \\
\hline zenotravel(20) & 11 & 11 & $11 \pm 0$ & 11 & 11 & $11 \pm 0$ \\
\hline
\end{tabular}

Table A.5: Coverage comparison (the number of instances solved in 5min, 4GB, LMcut heuristics) on 1104 standard IPC benchmark instances. We highlight the best results when the difference between the maximum and the minimum coverage exceeds 2 . 


\begin{tabular}{|c|c|c|c|c|c|c|}
\hline & 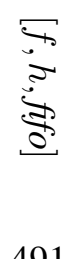 & 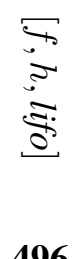 & 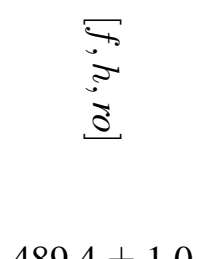 & 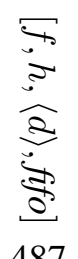 & 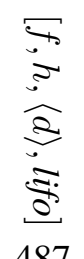 & 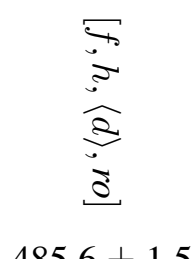 \\
\hline IPC benchmark (1104) & 491 & 496 & $489.4 \pm 1.0$ & 487 & 487 & $485.6 \pm 1.5$ \\
\hline airport(50) & 9 & 9 & $9 \pm 0$ & 9 & 9 & $9 \pm 0$ \\
\hline barman-opt11(20) & 4 & 4 & $4 \pm 0$ & 4 & 4 & $4 \pm 0$ \\
\hline $\operatorname{blocks}(35)$ & 22 & 22 & $22 \pm 0$ & 22 & 21 & $21.9 \pm 0.3$ \\
\hline cybersec(19) & 0 & 0 & $0 \pm 0$ & 0 & 0 & $0 \pm 0$ \\
\hline $\operatorname{depot}(22)$ & 6 & 6 & $5 \pm 0$ & 5 & 5 & $5 \pm 0$ \\
\hline driverlog $(20)$ & 12 & 12 & $12 \pm 0$ & 12 & 12 & $12 \pm 0$ \\
\hline elevators-opt11(20) & 13 & 13 & $13 \pm 0$ & 12 & 12 & $12 \pm 0$ \\
\hline floortile-opt11(20) & 6 & 6 & $6 \pm 0$ & 6 & 6 & $6 \pm 0$ \\
\hline freecell(80) & 17 & 17 & $16 \pm 0$ & 16 & 16 & $16 \pm 0$ \\
\hline $\operatorname{grid}(5)$ & 2 & 2 & $2 \pm 0$ & 2 & 2 & $2 \pm 0$ \\
\hline gripper(20) & 20 & 20 & $20 \pm 0$ & 20 & 20 & $20 \pm 0$ \\
\hline hanoi(30) & 14 & 14 & $14 \pm 0$ & 14 & 14 & $14 \pm 0$ \\
\hline logistics00(28) & 20 & 20 & $20 \pm 0$ & 20 & 20 & $20 \pm 0$ \\
\hline $\operatorname{miconic}(150)$ & 73 & 73 & $73.2 \pm 0.4$ & 73 & 73 & $72.2 \pm 0.4$ \\
\hline mprime(35) & 23 & 24 & $23.7 \pm 0.5$ & 23 & 24 & $23.4 \pm 0.5$ \\
\hline mystery(30) & 15 & 16 & $15 \pm 0$ & 15 & 16 & $15 \pm 0$ \\
\hline nomystery-opt11(20) & 18 & 18 & $18 \pm 0$ & 18 & 18 & $18 \pm 0$ \\
\hline openstacks-opt11(20) & 15 & 19 & $15.4 \pm 0.5$ & 19 & 19 & $\mathbf{1 9} \pm 0$ \\
\hline parcprinter-opt11(20) & 10 & 10 & $10 \pm 0$ & 10 & 10 & $10 \pm 0$ \\
\hline parking-opt $11(20)$ & 1 & 1 & $1 \pm 0$ & 1 & 1 & $1 \pm 0$ \\
\hline pathways(30) & 4 & 4 & $4 \pm 0$ & 4 & 4 & $4 \pm 0$ \\
\hline pegsol-opt11(20) & 19 & 19 & $19 \pm 0$ & 19 & 19 & $19 \pm 0$ \\
\hline pipesworld-notankage(50) & 10 & 10 & $9.9 \pm 0.3$ & 10 & 9 & $9.8 \pm 0.4$ \\
\hline pipesworld-tankage(50) & 13 & 13 & $13.2 \pm 0.4$ & 13 & 13 & $13 \pm 0$ \\
\hline psr-small(50) & 50 & 50 & $50 \pm 0$ & 50 & 50 & $50 \pm 0$ \\
\hline $\operatorname{rovers}(40)$ & 8 & 8 & $8 \pm 0$ & 8 & 8 & $7.1 \pm 0.3$ \\
\hline scanalyzer-opt11(20) & 10 & 10 & $10 \pm 0$ & 10 & 10 & $10 \pm 0$ \\
\hline sokoban-opt11(20) & 20 & 20 & $20 \pm 0$ & 19 & 19 & $19 \pm 0$ \\
\hline storage (30) & 15 & 15 & $15 \pm 0$ & 15 & 15 & $15 \pm 0$ \\
\hline tidybot-opt11(20) & 0 & 0 & $0 \pm 0$ & 0 & 0 & $0 \pm 0$ \\
\hline $\operatorname{tpp}(30)$ & 7 & 6 & $6 \pm 0$ & 6 & 6 & $6 \pm 0$ \\
\hline transport-opt11(20) & 7 & 7 & $7 \pm 0$ & 6 & 6 & $6 \pm 0$ \\
\hline visitall-opt11(20) & 9 & 9 & $9 \pm 0$ & 9 & 9 & $9 \pm 0$ \\
\hline woodworking-opt11(20) & 7 & 7 & $7 \pm 0$ & 7 & 7 & $7 \pm 0$ \\
\hline zenotravel(20) & 12 & 12 & $\mathbf{1 2} \pm 0$ & 10 & 10 & $10.1 \pm 0.3$ \\
\hline
\end{tabular}

Table A.6: Coverage comparison (the number of instances solved in 5min, 4GB, M\&S heuristics) on 1104 standard IPC benchmark instances. We highlight the best results when the difference between the maximum and the minimum coverage exceeds 2 . 


\section{A.3 Additional Figures for Figure 6.1; lifo Default Tiebreaking}
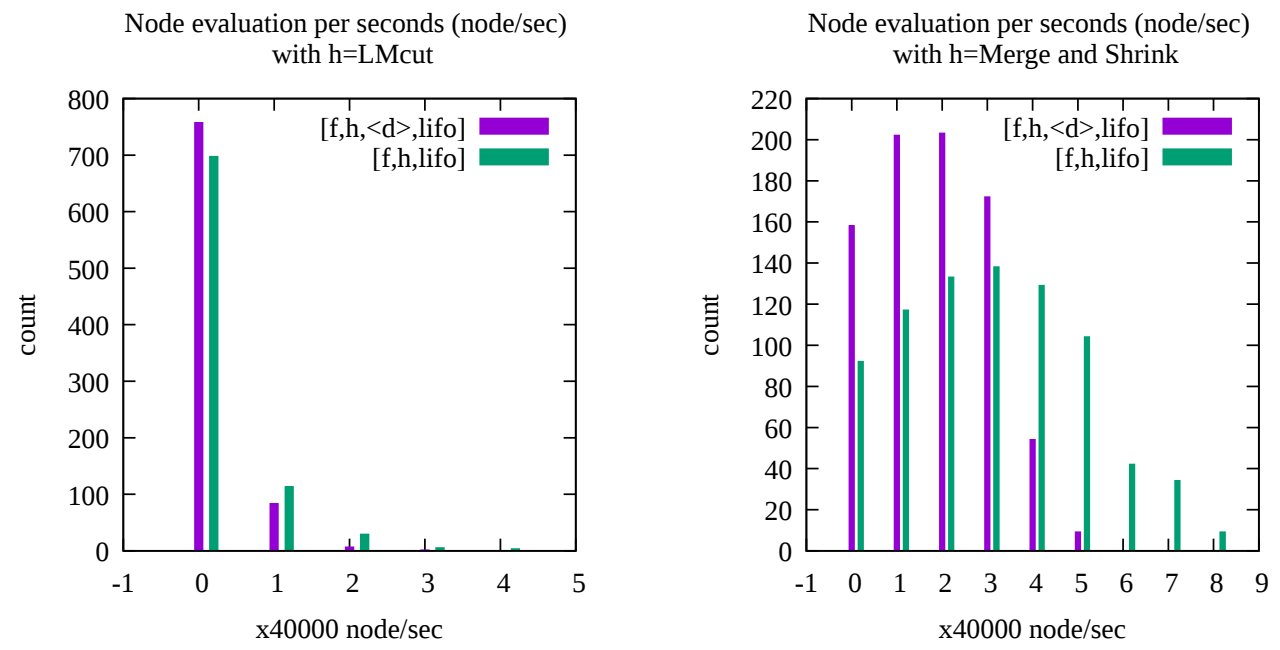

Figure A.1: Histogram comparing the node evaluation ratio (node/sec) between standard tiebreaking $([f, h, l i f o])$ and depth-based tie-breaking $([f, h,\langle d\rangle$, lifo $])$ on LMcut and $M \& S$ heuristics. On M\&S, compared to LMcut, node evaluation rate more often becomes slower when depth is enabled. This is because the node evaluation of $M \& S$ is an order of magnitude faster than LMcut, and the overhead of managing depth-based tie-breaking queue becomes significant. 


\section{A.4 Additional Figures for Figure 6.3: More Histograms for the Size of Final Plateaus}

These includes 12 additional histograms for the size of final plateaus on more variety of domains and instances.
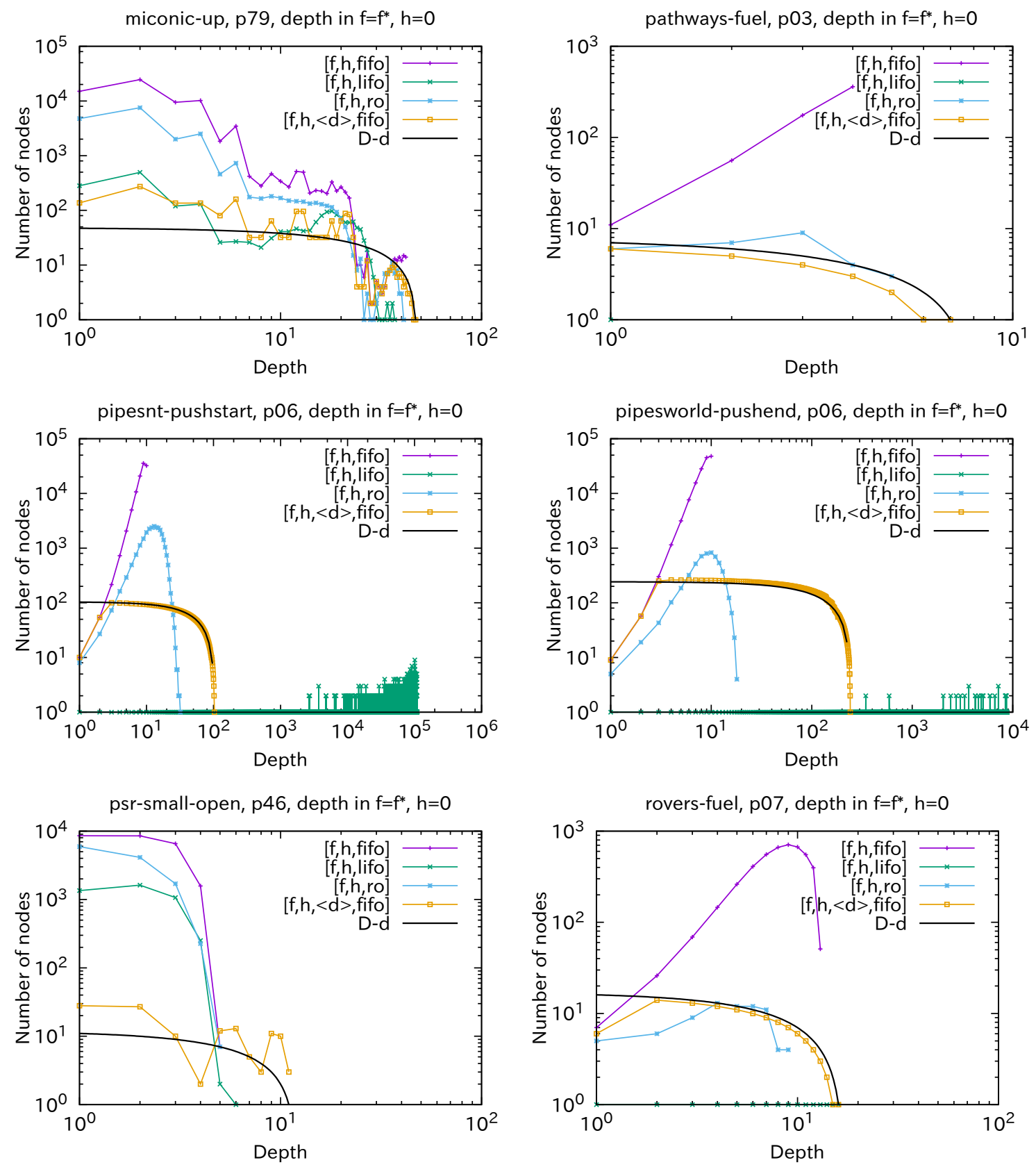

Figure A.2: (Page 1/2) Number of nodes ( $y$-axis) expanded per depth ( $x$-axis) in the final plateau with different tie-breaking strategies. Both axes are in logarithmic scale. 
scanalyzer-analyze, $\mathrm{p} 04$, depth in $\mathrm{f}=\mathrm{f}^{*}, \mathrm{~h}=0$

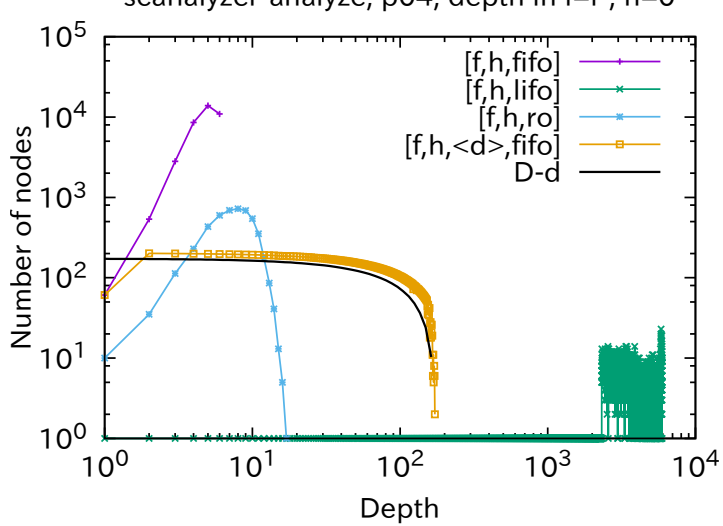

tidybot-motion, $\mathrm{p} 16$, depth in $\mathrm{f}=\mathrm{f}^{*}, \mathrm{~h}=0$

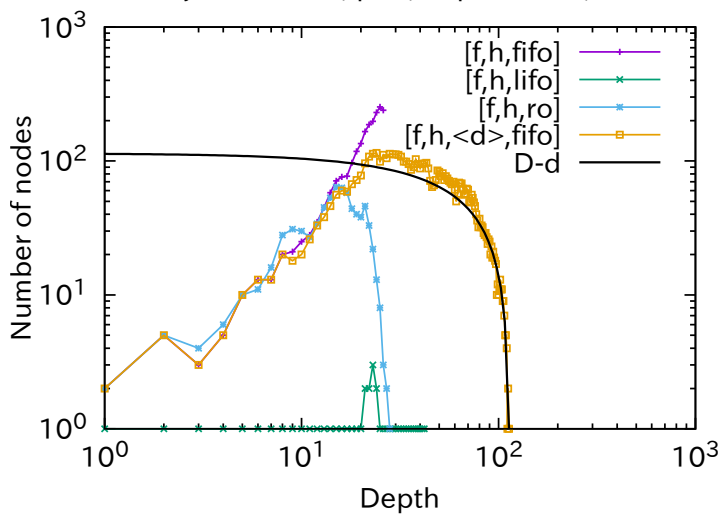

woodworking-cut, $\mathrm{p04}$, depth in $\mathrm{f}=\mathrm{f}^{*}, \mathrm{~h}=0$



storage-lift, $\mathrm{p} 11$, depth in $\mathrm{f}=\mathrm{f}^{*}, \mathrm{~h}=0$

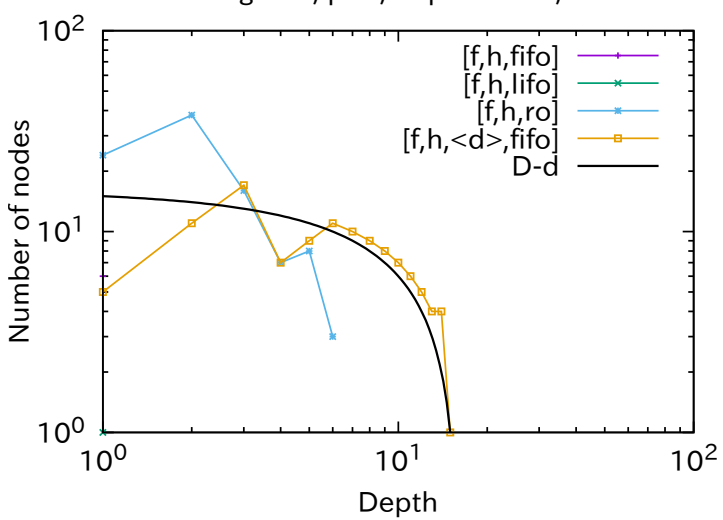

tpp-fuel, $\mathrm{p08}$, depth in $\mathrm{f}=\mathrm{f}^{*}, \mathrm{~h}=0$

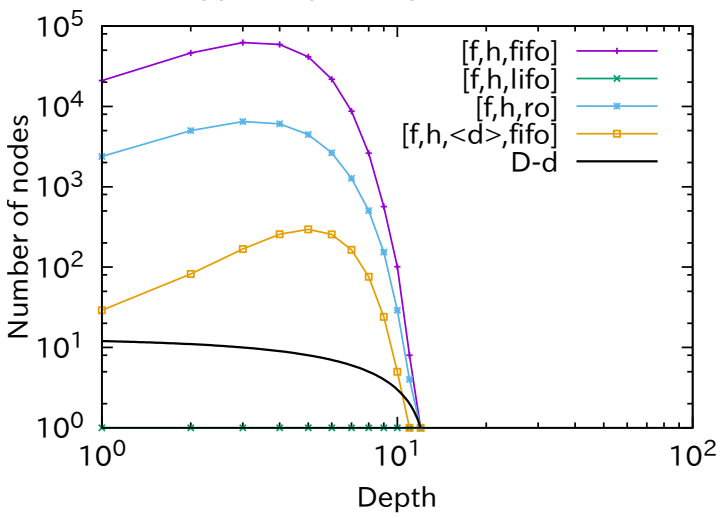

zenotravel-fuel, p07, depth in $\mathrm{f}=\mathrm{f}^{*}, \mathrm{~h}=0$

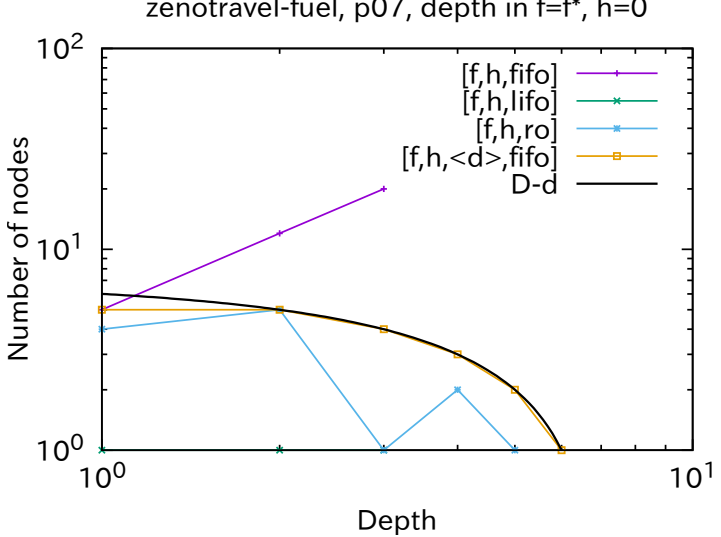

Figure A.3: (Page 2/2) Number of nodes ( $y$-axis) expanded per depth ( $x$-axis) in the final plateau with different tie-breaking strategies. Both axes are in logarithmic scale. 


\section{A.5 Additional Figures for Figure 6.4: More Histograms for the Size of Non-final Plateaus}

These are the additional histograms for the size of non-final plateaus on more variety of domains and instances.
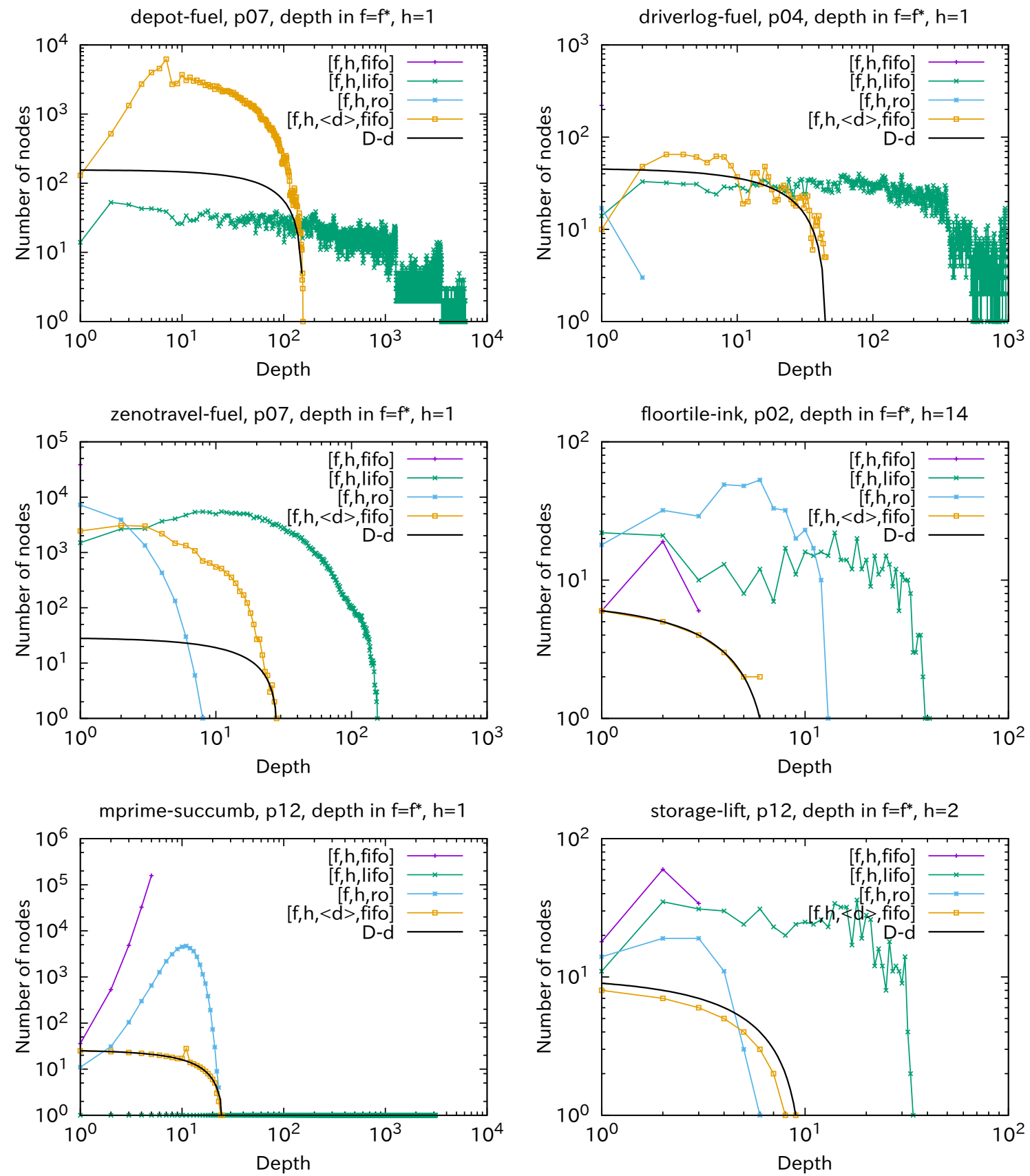

Figure A.4: Depth distribution in the non-final plateaus (plateau $\left.\left(f^{*}, h\right), h \neq 0\right)$ : Other domains. 


\section{A.6 Detailed Data for Table 8.2}

\begin{tabular}{|c|c|c|c|c|c|c|c|c|c|c|c|c|}
\hline & & & $\begin{array}{l}\text { to } \\
\overrightarrow{3} \\
\overrightarrow{3}\end{array}$ & & ") & वे & &  & $\begin{array}{l}\overrightarrow{5} \\
2 \\
\text { ग } \\
\overrightarrow{0} \\
\overrightarrow{0}\end{array}$ & 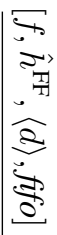 & 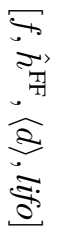 & 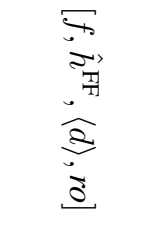 \\
\hline Zerocost (620) & 2 & & $1 .($ & 305 & & $305.9 \pm 2$. & 337 & 40 & \pm 2.2 & 340 & 42 & $=1.8$ \\
\hline airpo & 13 & 12 & 2.7 & 14 & 12 & $12.8 \pm 0.8$ & 13 & 11 & $11.7 \pm 0.5$ & 13 & 11 & $11.7 \pm 0.5$ \\
\hline blocks-stack(20) & 15 & 15 & 5.0 & 15 & 15 & $15 \pm 0$ & 17 & 17 & $17 \pm 0$ & 17 & 17 & $17 \pm 0$ \\
\hline depot-fuel(22) & 6 & 6 & 6.0 & 6 & 6 & $6 \pm 0$ & 6 & 6 & $6 \pm 0$ & 6 & 6 & $6 \pm 0$ \\
\hline driverlog-fuel(20) & 8 & 8 & 8.0 & 8 & 8 & $8 \pm 0$ & 8 & 8 & $8 \pm 0$ & 8 & 8 & $8 \pm 0$ \\
\hline elevators-up $(20)$ & 20 & 20 & 19.9 & 20 & 20 & $\mathbf{2 0} \pm 0$ & 20 & 20 & $\mathbf{2 0} \pm 0$ & 20 & 20 & $\mathbf{2 0} \pm 0$ \\
\hline floortile-ink (20) & 8 & 8 & 8.0 & 8 & 8 & $8 \pm 0$ & 9 & 8 & $8.7 \pm 0.5$ & 9 & 8 & $8.7 \pm 0.5$ \\
\hline freecell-move(20) & 12 & 14 & 13.3 & 12 & 14 & $13.2 \pm 0.4$ & 17 & 18 & $17.9 \pm 0.8$ & 17 & 18 & $18.3 \pm 0.9$ \\
\hline grid-fuel(5) & 1 & 1 & 1.0 & 1 & 1 & $1 \pm 0$ & 1 & 1 & $1 \pm 0$ & 1 & 1 & $1 \pm 0$ \\
\hline gripper-move(20) & 6 & 6 & 6.0 & 6 & 6 & $6 \pm 0$ & 6 & 6 & $6 \pm 0$ & 6 & 6 & $6 \pm 0$ \\
\hline hikin & 8 & 8 & 8.0 & 8 & 8 & $8 \pm 0$ & 9 & 9 & $9 \pm 0$ & 9 & 9 & $9 \pm 0$ \\
\hline logistics00-fuel(28) & 15 & 15 & 15.0 & 15 & 15 & $15 \pm 0$ & 15 & 15 & $15 \pm 0$ & 15 & 15 & $15 \pm 0$ \\
\hline miconic-up(30) & 14 & 17 & 15.1 & 14 & 17 & $15.1 \pm 0.9$ & 15 & 21 & $17.9 \pm 1.2$ & 15 & 21 & $18 \pm 1.2$ \\
\hline mprime-succumb(35) & 19 & 16 & 19.1 & 20 & 16 & $20.1 \pm 0.6$ & 30 & 23 & $28.3 \pm 0.9$ & 30 & 27 & $29.3 \pm 0.7$ \\
\hline mystery-feast(20) & 7 & 6 & 6.9 & 6 & 5 & $5.9 \pm 0.3$ & 8 & 8 & $\mathbf{8} \pm 0$ & 8 & 8 & $8 \pm 0$ \\
\hline nomystery & 10 & 10 & 10.0 & 10 & 10 & $10 \pm 0$ & 10 & 10 & $10 \pm 0$ & 10 & 10 & $10 \pm 0$ \\
\hline parking-movecc $(20)$ & 13 & 14 & 14.3 & 13 & 15 & $14.4 \pm 1.5$ & 20 & 20 & $\mathbf{2 0} \pm 0$ & 20 & 20 & $\mathbf{2 0} \pm 0$ \\
\hline pathway & 5 & 5 & 4.1 & 5 & 5 & $4 \pm 0$ & 5 & 5 & $5 \pm 0$ & 5 & 5 & $5 \pm 0$ \\
\hline pipesnt-pushstart(20) & 7 & 8 & 7.7 & 8 & 8 & $7.8 \pm 0.4$ & 9 & 9 & $9 \pm 0$ & 9 & 9 & $9 \pm 0$ \\
\hline pipesworld-pushend(20) & 5 & 6 & 5.1 & 5 & 5 & $5 \pm 0$ & 7 & 8 & $7.1 \pm 0.3$ & 7 & 7 & $7.7 \pm 0.5$ \\
\hline psr-small-open(20) & 19 & 19 & 19.0 & 19 & 19 & $19 \pm 0$ & 19 & 19 & $19 \pm 0$ & 19 & 19 & $19 \pm 0$ \\
\hline rovers-fuel(40) & 7 & 7 & 7.0 & 7 & 7 & $7 \pm 0$ & 8 & 9 & $8 \pm 0$ & 8 & 8 & $8 \pm 0$ \\
\hline scanalyzer-analyze(20) & 8 & 11 & 10.1 & 16 & 18 & $15.3 \pm 0.9$ & 15 & 15 & $15 \pm 0$ & 15 & 15 & $15 \pm 0$ \\
\hline sokoban-pushgoal(20) & 16 & 16 & 16.0 & 16 & 16 & $16 \pm 0$ & 17 & 17 & $17 \pm 0$ & 17 & 17 & $17 \pm 0$ \\
\hline storage-lift(20) & 4 & 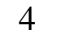 & 4.0 & 4 & 4 & $4 \pm 0$ & 4 & 4 & $4.3 \pm 0.5$ & 4 & 4 & $4.8 \pm 0.4$ \\
\hline tidybot-motion(20) & 14 & 14 & 14.0 & 14 & 14 & $14 \pm 0$ & 15 & 16 & $16 \pm 0$ & 16 & 16 & $15.9 \pm 0.3$ \\
\hline tpp-fuel(30) & 8 & 10 & 8.7 & 8 & 10 & $8.2 \pm 0.4$ & 8 & 10 & $9.1 \pm 0.3$ & 10 & 10 & $10 \pm 0$ \\
\hline woodworking-cut(20) & 20 & 20 & 20.0 & 20 & 20 & $\mathbf{2 0} \pm 0$ & 19 & 20 & $\mathbf{2 0} \pm 0$ & 19 & 20 & $\mathbf{2 0} \pm 0$ \\
\hline zenotravel-fuel(20) & 7 & 7 & 7.0 & 7 & 7 & $7 \pm 0$ & 7 & 7 & $7 \pm 0$ & 7 & 7 & $7 \pm 0$ \\
\hline
\end{tabular}

Table A.7: Coverage results with LMcut for computing $f$ and inadmissible distance-to-go heuristics for tie-breaking, on 620 Zerocost instances. We highlight the best results when the difference between the maximum and the minimum coverage exceeds 2 , over all configurations including Table A.3. 


\begin{tabular}{|c|c|c|c|c|c|c|c|c|c|c|c|c|}
\hline & 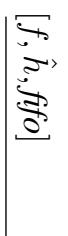 &  & 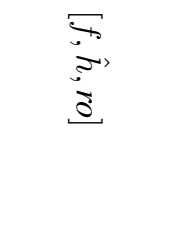 & 尔 & స్ &  & 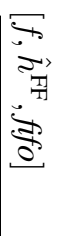 & 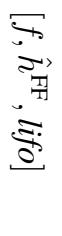 & 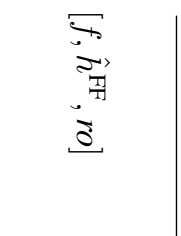 & 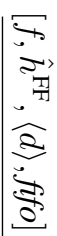 & 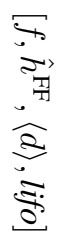 & 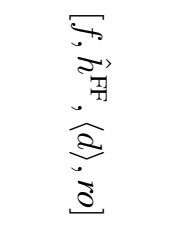 \\
\hline Zerocost (620) & 308 & & $173+1$ & 30 & 306 & $7.8 \pm 1.4$ & 33 & & $337.9 \pm 2.1$ & 37 & 333 & $=1.3$ \\
\hline airport-fuel(20) & 1 & 1 & $1 \pm 0$ & 1 & 1 & $1 \pm 0$ & 5 & 5 & $5 \pm 0$ & 5 & 5 & $5 \pm 0$ \\
\hline blocks-stack(20) & 20 & 20 & $20 \pm 0$ & 20 & 20 & $20 \pm 0$ & 20 & 19 & $19.9 \pm 0.3$ & 20 & 20 & $19.9 \pm 0.3$ \\
\hline depot-fuel(22) & 6 & 6 & $6 \pm 0$ & 6 & 6 & $6 \pm 0$ & 4 & 4 & $4 \pm 0$ & 4 & 4 & $4 \pm 0$ \\
\hline driverlog-fuel(20) & 9 & 9 & $9 \pm 0$ & 9 & 9 & $9 \pm 0$ & 9 & 9 & $9 \pm 0$ & 9 & 9 & $9 \pm 0$ \\
\hline elevators-up $(20)$ & 19 & 19 & $19 \pm 0$ & 19 & 19 & $19 \pm 0$ & 20 & 20 & $\mathbf{2 0} \pm 0$ & 20 & 20 & $\mathbf{2 0} \pm 0$ \\
\hline floortile-ink(20) & 8 & 8 & $8 \pm 0$ & 8 & 8 & $8 \pm 0$ & 9 & 8 & $8.8 \pm 0.4$ & 9 & 8 & $8.8 \pm 0.4$ \\
\hline freecell-move(20) & 13 & 14 & $12.7 \pm 0.7$ & 13 & 13 & $12.7 \pm 0.7$ & 17 & 17 & $17.4 \pm 0.5$ & 17 & 17 & $17.3 \pm 0.7$ \\
\hline grid-fuel(5) & 2 & 2 & $2 \pm 0$ & 2 & 2 & $2 \pm 0$ & 2 & 2 & $2 \pm 0$ & 2 & 2 & $2 \pm 0$ \\
\hline gripper-move(20) & 20 & 20 & $20 \pm 0$ & 20 & 20 & $20 \pm 0$ & 20 & 20 & $20 \pm 0$ & 20 & 20 & $20 \pm 0$ \\
\hline hiking-fuel(20) & 13 & 13 & $12.1 \pm 0.3$ & 13 & 13 & $12.1 \pm 0.3$ & 11 & 11 & $11 \pm 0$ & 11 & 11 & $11 \pm 0$ \\
\hline logisticsO0-fuel(28) & 16 & 16 & $16 \pm 0$ & 16 & 16 & $16 \pm 0$ & 16 & 16 & $16 \pm 0$ & 16 & 16 & $16 \pm 0$ \\
\hline miconic-up $(30)$ & 22 & 22 & $22 \pm 0$ & 22 & 22 & $22.1 \pm 0.3$ & 30 & 30 & $30 \pm 0$ & 30 & 30 & $30 \pm 0$ \\
\hline mprime-succumb $(35)$ & 21 & 17 & $20.4 \pm 0.7$ & 21 & 17 & $20.4 \pm 0.7$ & 28 & 23 & $27.4 \pm 0.7$ & 28 & 25 & $27.7 \pm 0.7$ \\
\hline mystery-feast(20) & 5 & 5 & $5 \pm 0$ & 5 & 5 & $5 \pm 0$ & 3 & 3 & $3 \pm 0$ & 3 & 3 & $3 \pm 0$ \\
\hline nomystery-fuel(20) & 16 & 16 & $16 \pm 0$ & 16 & 16 & $16 \pm 0$ & 15 & 15 & $15 \pm 0$ & 15 & 15 & $15 \pm 0$ \\
\hline parking-movecc $(20)$ & 2 & 2 & $2 \pm 0$ & 2 & 2 & $2 \pm 0$ & 10 & 10 & $\mathbf{1 0 . 3} \pm 1.0$ & 10 & 10 & $\mathbf{1 0 . 3} \pm 1.0$ \\
\hline pathways-fuel(30) & 4 & 4 & $4 \pm 0$ & 4 & 4 & $4 \pm 0$ & 4 & 4 & $4 \pm 0$ & 4 & 4 & $4 \pm 0$ \\
\hline pipesnt-pushstart(20) & 1 & 2 & $1.9 \pm 0.8$ & 1 & 2 & $1.8 \pm 0.7$ & 5 & 5 & $5 \pm 0$ & 5 & 5 & $5 \pm 0$ \\
\hline pipesworld-pushend(20) & 8 & 7 & $7.8 \pm 0.4$ & 8 & 8 & $8 \pm 0$ & 5 & 5 & $5.4 \pm 0.7$ & 5 & 5 & $5.6 \pm 0.5$ \\
\hline psr-small-open(20) & 19 & 19 & $19 \pm 0$ & 19 & 19 & $19 \pm 0$ & 19 & 19 & $19 \pm 0$ & 19 & 19 & $19 \pm 0$ \\
\hline rovers-fuel(40) & 8 & 8 & $8 \pm 0$ & 8 & 8 & $8 \pm 0$ & 8 & 8 & $8 \pm 0$ & 8 & 8 & $8 \pm 0$ \\
\hline $\operatorname{scanalyzer-analyze}(20)$ & 15 & 14 & $15 \pm 0$ & 14 & 15 & $15 \pm 0$ & 15 & 16 & $\mathbf{1 5 . 4} \pm 0.7$ & 15 & 15 & $15.2 \pm 0.7$ \\
\hline sokoban-pushgoal(20) & 17 & 17 & $17 \pm 0$ & 17 & 17 & $17 \pm 0$ & 18 & 18 & $18.2 \pm 0.4$ & 18 & 18 & $18 \pm 0$ \\
\hline storage-lift $(20)$ & 4 & 4 & $4 \pm 0$ & 4 & 4 & $4 \pm 0$ & 4 & 4 & $4 \pm 0$ & 4 & 4 & $4 \pm 0$ \\
\hline tidybot-motion(20) & 0 & 0 & $0 \pm 0$ & 0 & 0 & $0 \pm 0$ & 0 & 0 & $0 \pm 0$ & 0 & 0 & $0 \pm 0$ \\
\hline tpp-fuel(30) & 9 & 10 & $9.4 \pm 0.5$ & 9 & 10 & $9.8 \pm 0.4$ & 10 & 11 & $10.9 \pm 0.3$ & 11 & 11 & $10.9 \pm 0.3$ \\
\hline woodworking-cut $(20)$ & 20 & 20 & $\mathbf{2 0} \pm 0$ & 20 & 20 & $\mathbf{2 0} \pm 0$ & 20 & 20 & $\mathbf{2 0} \pm 0$ & 20 & 20 & $\mathbf{2 0} \pm 0$ \\
\hline zenotravel-fuel $(20)$ & 10 & 10 & $10 \pm 0$ & 10 & 10 & $9.9 \pm 0.3$ & 9 & 9 & $9 \pm 0$ & 9 & 9 & $8.9 \pm 0.3$ \\
\hline
\end{tabular}

Table A.8: Coverage results with M\&S for computing $f$ and inadmissible distance-to-go heuristics for tie-breaking, on 620 Zerocost instances. We highlight the best results when the difference between the maximum and the minimum coverage exceeds 2 , over all configurations including Table A.4. 


\begin{tabular}{|c|c|c|c|c|c|c|c|c|c|c|c|c|}
\hline $\mathrm{Cl}$ & $\mid$ &  & $\begin{array}{l}\overline{0} \\
\text { 고 } \\
\overrightarrow{0}\end{array}$ & 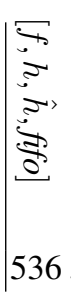 & 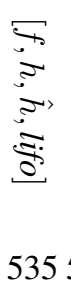 &  & 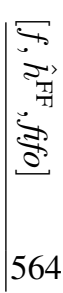 & 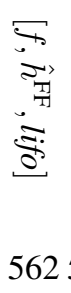 & 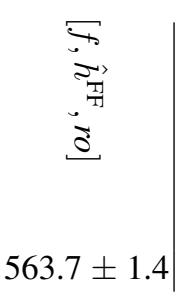 & 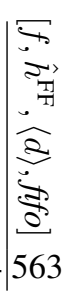 & 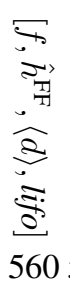 & 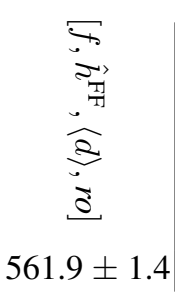 \\
\hline & 24 & & $9 \pm 0.6$ & 24 & 24 & $8 \pm 0.4$ & 25 & 24 & $8 \pm 0.4$ & 25 & 24 & . $0 \perp$ \\
\hline barman- & 0 & 0 & \pm 0 & 0 & 0 & $0 \pm 0$ & 0 & 0 & $0 \pm 0$ & 0 & 0 & $0 \pm 0$ \\
\hline & 27 & 27 & $27 \pm 0$ & 27 & 27 & $27 \pm 0$ & 27 & 27 & $27 \pm 0$ & 27 & 27 & $27 \pm 0$ \\
\hline $\mathrm{cyb}$ & 5 & 3 & \pm 1.2 & 6 & 4 & $5.4 \pm 0.7$ & 6 & 6 & $5.9 \pm 0.8$ & 6 & 5 & $6 \pm 0.7$ \\
\hline & 5 & 5 & \pm 0 & 5 & 5 & 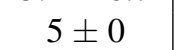 & 6 & 6 & \pm 0 & 6 & 6 & $6 \pm 0$ \\
\hline driv & 12 & 12 & $12 \pm 0$ & 12 & 12 & 0 & 13 & 13 & \pm 0 & 13 & 13 & $13 \pm 0$ \\
\hline elevato & 12 & 12 & 0 & 12 & 12 & 0 & 15 & 15 & .3 & 14 & 15 & \pm 0 \\
\hline & 6 & 6 & \pm 0 & 6 & 6 & +0 & 6 & 6 & & 6 &  & \pm 0 \\
\hline & 8 & 8 & 0 & 8 & 8 & & 9 & 9 & & 9 & 9 & \pm 0 \\
\hline & 1 & 1 & 0 & 1 & 1 & & 1 & 1 & & 1 & 1 & \pm 0 \\
\hline gripp & 6 & 6 & 0 & 6 & 6 & & 6 & 6 & & 6 & 6 & \pm 0 \\
\hline & 11 & 11 & $=0$ & 11 & 11 & & 12 & 12 & $=0$ & 12 & 12 & $11.9 \pm 0$ \\
\hline 10 & 17 & 17 & 0 & 17 & 17 & & 20 & 20 & & 20 & 20 & $20 \pm 0$ \\
\hline & 140 & 140 & \pm 0 & 140 & 140 & $=0$ & 140 & 140 & \pm 0 & 140 & 140 & $0 \pm 0$ \\
\hline & 20 & 21 & \pm 0.8 & 20 & 21 & 0.7 & 22 & 22 & \pm 0 & 22 & 22 & \pm 0 \\
\hline & 15 & 15 & \pm 0 & 15 & 15 & & 16 & 16 & & 16 & 16 & \pm 0 \\
\hline nomystery & 13 & 13 & 0 & 13 & 13 & & 14 & 14 & & 4 & 14 & \pm 0 \\
\hline & 10 & 10 & $=0$ & 10 & 10 & 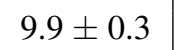 & 17 & 17 & & 7 & 17 & \pm 0 \\
\hline & 13 & 13 & \pm 0 & 13 & 13 & & 13 & 13 & 0 & 13 & 13 & \pm 0 \\
\hline & 1 & 1 & & 1 & 1 & & 1 & 1 & & & 1 & \pm 0 \\
\hline & 5 & 5 & & 5 & 5 & & 5 & 5 & &  & 5 & \pm 0 \\
\hline & 16 & 16 & $=0$ & 16 & 16 & & 17 & 17 & & 17 & 17 & $7 \pm 0$ \\
\hline j) & 12 & 12 & & 12 & 12 & & 13 & 13 & 0 & 13 & 13 & \pm 0 \\
\hline & 7 & 7 & & 7 & 7 & & 8 & 8 & & 8 & 8 & \pm 0 \\
\hline & 48 & 48 & $47.9 \pm 0.3$ & 48 & 48 & \pm 0 & 48 & 48 & $48 \pm 0$ & 48 & 48 & $48 \pm 0$ \\
\hline & 7 & 7 & & 7 & 7 & & 7 & 7 & & 7 & 7 & \pm 0 \\
\hline & 8 & 10 & & 10 & 10 & & 10 & 10 & & 10 & 10 & \pm 0 \\
\hline 6) & 17 & 17 & & 17 & 17 & & 19 & 19 & & 19 & 19 & \pm 0 \\
\hline & 14 & 14 & & 14 & 14 & & 14 & 14 & & 14 & 14 & \pm 0 \\
\hline tidybot-opt & 10 & 11 & $10.3 \pm 0.5$ & 11 & 11 & $10.6 \pm 0.5$ & 11 & 11 & $11 \pm 0$ & 11 & 11 & $11 \pm 0$ \\
\hline & 6 & 6 & & 6 & 6 & & 6 & 6 & & 6 & 6 & \pm 0 \\
\hline pt1 & 6 & 6 & & 6 & 6 & & 6 & 6 & & 6 & 6 & $6 \pm 0$ \\
\hline & 10 & 10 & & 10 & 10 & & 10 & 10 & & 10 & 10 & $10 \pm 0$ \\
\hline odworki & 11 & 8 & $9.3 \pm 1.0$ & 9 & 9 & $9 \pm 0$ & 10 & 9 & $10.1 \pm 1.1$ & 10 & 8 & $9.9 \pm 1.1$ \\
\hline zenotravel $(20)$ & 11 & 11 & $11 \pm 0$ & 11 & 11 & $11 \pm 0$ & 11 & 11 & $11 \pm 0$ & 11 & 11 & $11 \pm 0$ \\
\hline
\end{tabular}

Table A.9: Coverage results with LMcut for computing $f$ and inadmissible distance-to-go heuristics for tie-breaking, on 1104 standard IPC benchmark instances. 


\begin{tabular}{|c|c|c|c|c|c|c|c|c|c|c|c|c|}
\hline Cben & $\mid$ & ॠ &  & 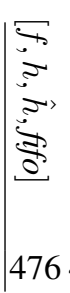 & 5 & 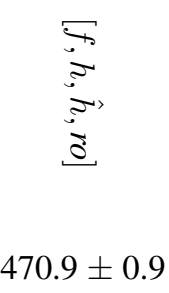 & 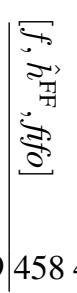 &  & 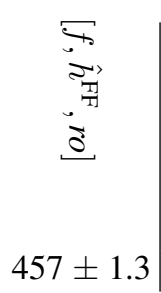 &  &  &  \\
\hline 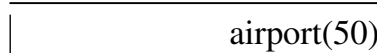 & 7 & 7 & \pm 0 & 7 & 7 & $7 \pm 0$ & 9 & 9 & $9 \pm 0$ & 9 & 9 & \pm 0 \\
\hline barman- & 4 & 4 & \pm 0 & 4 & 4 & \pm 0 & 4 & 4 & $4 \pm 0$ & 4 & 4 & \pm 0 \\
\hline & 22 & 21 & $=0$ & 21 & 21 & $21 \pm 0$ & 21 & 20 & \pm 0.3 & 20 & 20 & \pm 0 \\
\hline cyh & 0 & 0 & +0 & 0 & 0 & \pm 0 & 0 & 0 & \pm 0 & 0 & 0 & \pm 0 \\
\hline & 5 & 5 & & 5 & 5 & & 4 & 4 & & 4 & 4 & \pm 0 \\
\hline driv & 12 & 12 & $=0$ & 12 & 12 & $=0$ & 11 & 11 & & 11 & 11 & $1 \pm 0$ \\
\hline el & 13 & 13 & & 13 & 13 & & 10 & 10 & & 10 & 10 & \\
\hline & 6 & 6 & & 6 & 6 & & 7 & 7 & & 7 & 7 & \pm 0 \\
\hline & 15 & 15 & & 15 & 15 & & 14 & 14 & & 14 & 14 & \pm 0 \\
\hline & 2 & 2 & & 2 & 2 & & 2 & 2 & & 2 & 2 & \pm 0 \\
\hline gripp & 20 & 20 & & 20 & 20 & & 20 & 20 & & 20 & 20 & \pm 0 \\
\hline & 14 & 14 & & 14 & 14 & $=0$ & 13 & 13 & 0 & 13 & 13 & $3 \pm 0$ \\
\hline 10 & 20 & 20 & & 2 & 20 & & 20 & 20 & 0 & 20 & 20 & 0 \\
\hline & 72 & 72 & 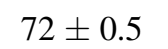 & 7 & 72 & 72 & 69 & 69 & 0.4 & 69 & 69 & + \\
\hline & 19 & 19 & 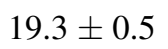 & 20 & 19 & .5 & 21 & 21 & 0.8 & 21 & 21 & \pm 0 \\
\hline & 15 & 15 & & 15 & 15 & & 15 & 15 & & 15 & 15 & 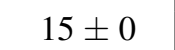 \\
\hline nomystery & 18 & 18 & & 1 & 18 & & 6 & 16 & & 6 & 16 & \pm 0 \\
\hline & 18 & 19 & & 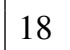 & 19 & & 18 & 18 & & 8 & 18 & $17.7 \pm 0$ \\
\hline & 10 & 10 & \pm 0 & 10 & 10 & 1 & 11 & 11 & \pm 0 & 11 & 11 & $1 \pm 0$ \\
\hline & 1 & 1 & 0.5 & 1 & 1 & 0. & 0 & 0 & & 0 & 0 & \pm 0 \\
\hline & 4 & 4 & & 4 & 4 & & 4 & 4 & & 4 & 4 & \pm 0 \\
\hline & 19 & 19 & & 19 & 19 & & 17 & 17 & & 17 & 17 & $7 \pm 0$ \\
\hline 0) & 6 & 5 & 0.7 & 6 & 5 & $=0.8$ & 9 & 9 & 0.5 & 9 & 9 & \pm 0.4 \\
\hline & 12 & 12 & & 12 & 12 & & 9 & 9 & & 9 & 9 & \pm 0 \\
\hline & 50 & 50 & $\llcorner 0$ & 50 & 50 & \pm 0 & 50 & 50 & \pm 0 & 50 & 50 & $50 \pm 0$ \\
\hline & 8 & 8 & & 7 & 8 & & 6 & 6 & & 6 & 6 & \pm 0 \\
\hline & 10 & 10 & & 10 & 10 & & 7 & 7 & & 1 & 7 & \pm 0.4 \\
\hline & 18 & 18 & & 18 & 18 & & 19 & 19 & & 19 & 19 & \pm 0 \\
\hline & 15 & 15 & & 15 & 15 & & 14 & 14 & & 14 & 14 & $14 \pm 0$ \\
\hline ybot-opt & 0 & 0 & & 0 & 0 & $=0$ & 0 & 0 & & 0 & 0 & \pm 0 \\
\hline & 6 & 6 & & 6 & 6 & & 6 & 6 & & 6 & 6 & \pm 0 \\
\hline )) & 7 & 7 & & 7 & 7 & \pm 0 & 6 & 6 & & 6 & 6 & \pm 0 \\
\hline & 9 & 9 & & 9 & 9 & & 9 & 9 & & 9 & 9 & $9 \pm 0$ \\
\hline odworki & 8 & 8 & \pm 0.3 & 8 & 8 & $8.1 \pm 0.3$ & 7 & 7 & $7.1 \pm 0.3$ & 7 & 7 & $7.1 \pm 0.3$ \\
\hline zenotravel(20) & 12 & 11 & $10.9 \pm 0.3$ & 12 & 11 & $10.9 \pm 0.3$ & 10 & 10 & $10 \pm 0$ & 10 & 10 & $10 \pm 0$ \\
\hline
\end{tabular}

Table A.10: Coverage results with M\&S for computing $f$ and inadmissible distance-to-go heuristics for tie-breaking, on 1104 standard IPC benchmark instances. 


\section{References}

Aghighi, M., \& Bäckström, C. (2015). Cost-optimal and Net-benefit Planning-A Parameterised Complexity View. In Proceedings of International Joint Conference on Artificial Intelligence (IJCAI).

Aghighi, M., \& Bäckström, C. (2016). A Multi-Parameter Complexity Analysis of Cost-Optimal and Net-Benefit Planning. In Proceedings of the International Conference of Automated Planning and Scheduling(ICAPS).

Amir, E., \& Engelhardt, B. (2003). Factored Planning. In Proceedings of International Joint Conference on Artificial Intelligence (IJCAI).

Asai, M., \& Fukunaga, A. (2015). Solving Large-Scale Planning Problems by Decomposition and Macro Generation. In Proceedings of the International Conference of Automated Planning and Scheduling(ICAPS), Jerusalem, Israel.

Asai, M., \& Fukunaga, A. (2016). Tiebreaking Strategies for Classical Planning Using $A^{*}$ Search. In Proceedings of AAAI Conference on Artificial Intelligence, Arizona, USA.

Benton, J., Talamadupula, K., Eyerich, P., Mattmüller, R., \& Kambhampati, S. (2010). G-Value Plateaus: A Challenge for Planning. In Proceedings of the International Conference of Automated Planning and Scheduling(ICAPS).

Boddy, M. S., Gohde, J., Haigh, T., \& Harp, S. A. (2005). Course of Action Generation for Cyber Security Using Classical Planning. In Proceedings of the International Conference of Automated Planning and Scheduling(ICAPS).

Botea, A., Enzenberger, M., Müller, M., \& Schaeffer, J. (2005). Macro-FF: Improving AI Planning with Automatically Learned Macro-Operators. J. Artif. Intell. Res.(JAIR), 24, 581-621.

Brafman, R. I., \& Domshlak, C. (2006). Factored Planning: How, When, and When Not. In Proceedings of AAAI Conference on Artificial Intelligence.

Burns, E. (2012). Heuristic search code library. https://github.com/eaburns/search.

Burns, E. A., Hatem, M., Leighton, M. J., \& Ruml, W. (2012). Implementing Fast Heuristic Search Code. In Proceedings of Annual Symposium on Combinatorial Search.

Chrpa, L., Vallati, M., \& McCluskey, T. L. (2015). On the Online Generation of Effective MacroOperators. In Proceedings of International Joint Conference on Artificial Intelligence (IJCAI).

Coles, A., \& Smith, A. (2007). Marvin: A Heuristic Search Planner with Online Macro-Action Learning. J. Artif. Intell. Res.(JAIR), 28, 119-156.

Cushing, W., Benton, J., \& Kambhampati, S. (2010). Cost Based Search Considered Harmful. In Proceedings of Annual Symposium on Combinatorial Search.

Dijkstra, E. W. (1959). A note on two problems in connexion with graphs. Numerische mathematik, $1(1), 269-271$.

Domshlak, C., Katz, M., \& Shleyfman, A. (2013). Symmetry Breaking: Satisficing Planning and Landmark Heuristics. In Proceedings of the International Conference of Automated Planning and Scheduling(ICAPS). 
Eyerich, P., Mattmüller, R., \& Röger, G. (2009). Using the Context-enhanced Additive Heuristic for Temporal and Numeric Planning. In Proceedings of the International Conference of Automated Planning and Scheduling(ICAPS).

Felner, A., Zahavi, U., Holte, R. C., Schaeffer, J., Sturtevant, N. R., \& Zhang, Z. (2011). Inconsistent heuristics in theory and practice. J. Artif. Intell. Res.(JAIR), 175(9-10), 1570-1603.

Fink, A., \& Voss, S. (1999). Applications of Modern Heuristic Search Methods to Pattern Sequencing Problems. Computers \& Operations Research, 26(1), 17-34.

Fox, M., \& Long, D. (1998). The Automatic Inference of State Invariants in TIM. J. Artif. Intell. Res.(JAIR), 9, 367-421.

Gerevini, A. E., Saetti, A., \& Vallati, M. (2009). An Automatically Configurable Portfolio-based Planner with Macro-actions: PbP. In Proceedings of the International Conference of Automated Planning and Scheduling(ICAPS).

Hall, D. L. W., Cohen, A., Burkett, D., \& Klein, D. (2013). Faster Optimal Planning with PartialOrder Pruning. In Proceedings of the International Conference of Automated Planning and Scheduling(ICAPS).

Hansen, E. A., \& Zhou, R. (2007). Anytime Heuristic Search. J. Artif. Intell. Res.(JAIR), 28, 267-297.

Hart, P. E., Nilsson, N. J., \& Raphael, B. (1968). A Formal Basis for the Heuristic Determination of Minimum Cost Paths. Systems Science and Cybernetics, IEEE Transactions on, 4(2), 100107.

Helmert, M. (2006). The Fast Downward Planning System. J. Artif. Intell. Res.(JAIR), 26, 191-246.

Helmert, M., \& Domshlak, C. (2009). Landmarks, Critical Paths and Abstractions: What's the Difference Anyway?. In Proceedings of the International Conference of Automated Planning and Scheduling(ICAPS).

Helmert, M., Haslum, P., Hoffmann, J., \& Nissim, R. (2014). Merge-and-Shrink Abstraction: A Method for Generating Lower Bounds in Factored State Spaces. Journal of ACM, 61(3), 16:1-16:63.

Hoffmann, J. (2005). Where 'Ignoring Delete Lists' Works: Local Search Topology in Planning Benchmarks. J. Artif. Intell. Res.(JAIR), 24, 685-758.

Hoffmann, J. (2011). Analyzing Search Topology Without Running Any Search: On the Connection Between Causal Graphs and $h^{+}$. J. Artif. Intell. Res.(JAIR), 41(2), 155-229.

Hoffmann, J., \& Nebel, B. (2001). The FF Planning System: Fast Plan Generation through Heuristic Search. J. Artif. Intell. Res.(JAIR), 14, 253-302.

Holte, R. C. (2010). Common Misconceptions Concerning Heuristic Search. In Proceedings of Annual Symposium on Combinatorial Search.

Imai, T., \& Kishimoto, A. (2011). A Novel Technique for Avoiding Plateaus of Greedy Best-First Search in Satisficing Planning. In Proceedings of AAAI Conference on Artificial Intelligence.

Kautz, H. A., \& Selman, B. (1992). Planning as Satisfiability.. In ECAI, Vol. 92, pp. 359-363.

Korf, R. E. (1985). Depth-First Iterative-Deepening: An Optimal Admissible Tree Search. Artificial Intelligence, 27(1), 97-109. 
Korf, R. E. (1999). Divide-and-Conquer Bidirectional Search: First Results. In Proceedings of International Joint Conference on Artificial Intelligence (IJCAI).

Korf, R. E., \& Zhang, W. (2000). Divide-and-Conquer Frontier Search Applied to Optimal Sequence Alignment. In Proceedings of AAAI Conference on Artificial Intelligence.

Likhachev, M., Ferguson, D., Gordon, G., Stentz, A., \& Thrun, S. (2008). Anytime search in dynamic graphs. Artificial Intelligence, 172(14), 1613-1643.

Nakhost, H., \& Müller, M. (2009). Monte-Carlo Exploration for Deterministic Planning. In Proceedings of International Joint Conference on Artificial Intelligence (IJCAI).

Nilsson, N. J. (1971). Problem Solving Methods in Artificial Intelligence. McGraw-Hill.

Pearl, J. (1984). Heuristics: Intelligent Search Strategies for Computer Problem Solving. AddisonWesley Pub. Co., Inc., Reading, MA.

Pearl, J., \& Kim, J. H. (1982). Studies in Semi-Admissible Heuristics. Pattern Analysis and Machine Intelligence, IEEE Transactions on, 4(4), 392-399.

Pochter, N., Zohar, A., \& Rosenschein, J. S. (2011). Exploiting Problem Symmetries in State-Based Planners. In Proceedings of AAAI Conference on Artificial Intelligence.

Pohl, I. (1973). The avoidance of (relative) catastrophe, heuristic competence, genuine dynamic weighting and computational issues in heuristic problem solving. In Proceedings of International Joint Conference on Artificial Intelligence (IJCAI).

Richter, S., \& Westphal, M. (2010). The LAMA Planner: Guiding Cost-Based Anytime Planning with Landmarks. J. Artif. Intell. Res.(JAIR), 39(1), 127-177.

Richter, S., Westphal, M., \& Helmert, M. (2011). LAMA 2008 and 2011. In Proceedings of the 7th International Planning Competition (IPC), pp. 117-124.

Röger, G., \& Helmert, M. (2010). The More, the Merrier: Combining Heuristic Estimators for Satisficing Planning. In Proceedings of the International Conference of Automated Planning and Scheduling(ICAPS).

Stern, R., Kulberis, T., Felner, A., \& Holte, R. C. (2010). Using Lookaheads with Optimal Best-First Search. In Proceedings of AAAI Conference on Artificial Intelligence.

Thayer, J. T., \& Ruml, W. (2009). Using Distance Estimates in Heuristic Search. In Proceedings of the International Conference of Automated Planning and Scheduling(ICAPS).

Thayer, J. T., \& Ruml, W. (2011). Bounded Suboptimal Search: A Direct Approach using Inadmissible Estimates. In Proceedings of International Joint Conference on Artificial Intelligence (IJCAI).

Valenzano, R. A., Schaeffer, J., Sturtevant, N., \& Xie, F. (2014). A Comparison of KnowledgeBased GBFS Enhancements and Knowledge-Free Exploration. In Proceedings of the International Conference of Automated Planning and Scheduling(ICAPS).

Valenzano, R. A., \& Xie, F. (2016). On the Completeness of BestFirst Search Variants that Use Random Exploration. In Proceedings of AAAI Conference on Artificial Intelligence.

Vallati, M., Hutter, F., Chrpa, L., \& McCluskey, T. L. (2015). On the Effective Configuration of Planning Domain Models. In Proceedings of International Joint Conference on Artificial Intelligence (IJCAI). 
van den Briel, M., \& Kambhampati, S. (2005). Optiplan: Unifying IP-based and Graph-based Planning. J. Artif. Intell. Res.(JAIR), 24, 919-931.

Wehrle, M., Helmert, M., Alkhazraji, Y., \& Mattmüller, R. (2013). The Relative Pruning Power of Strong Stubborn Sets and Expansion Core. In Proceedings of the International Conference of Automated Planning and Scheduling(ICAPS).

Wilt, C. M., \& Ruml, W. (2011). Cost-Based Heuristic Search is Sensitive to the Ratio of Operator Costs. In Proceedings of Annual Symposium on Combinatorial Search.

Wray, K. H., Zilberstein, S., \& Mouaddib, A.-I. (2015). Multi-Objective MDPs with Conditional Lexicographic Reward Preferences. In Proceedings of AAAI Conference on Artificial Intelligence, pp. 3418-3424.

Xie, F., Müller, M., Holte, R. C., \& Imai, T. (2014). Type-Based Exploration with Multiple Search Queues for Satisficing Planning. In Proceedings of AAAI Conference on Artificial Intelligence. 